\title{
A Convenient Solvothermal Synthesis of Group Six PNP Pincer Tricarbonyl Complexes
}

\author{
Matthias Mastalir, ${ }^{a}$ Sara R. M. M. de Aguiar, ${ }^{a}$ Mathias Glatz,, Berthold Stöger, ${ }^{\text {b }}$ \\ Karl Kirchner*, a
}

a Institute of Applied Synthetic Chemistry, Vienna University of Technology, and b Institute of Chemical Technologies and Analytics, Vienna University of Technology, Getreidemarkt 9, A-1060 Vienna, AUSTRIA

\section{Supporting Information}

\section{Experimental Section}

Synthesis. 2-Chloro-1,3-bis[(S)-1-phenylethyl]-1,3,2-diazaphospholidine (SPEA-PCl)<smiles>CC(c1ccccc1)N1CCN(C(C)c2ccccc2)C1Cl</smiles>

SPEA-PCI

N,N'-Bis[(1S)-1-phenylethyl]-1,2-ethanediamine $(5 \mathrm{~g}, 18.63 \mathrm{mmol})^{1}$ and phosphorus trichloride $(2.56 \mathrm{~g}$, $18.63 \mathrm{mmol}$ ) were added via syringe within $1 \mathrm{~min}$ to a stirred solution of $\mathrm{N}, \mathrm{N}$-diisopropylethylamine (DIPEA) $(5.06 \mathrm{~g}, 39.12 \mathrm{mmol})$ in toluene $(35 \mathrm{~mL})$ at $0{ }^{\circ} \mathrm{C}$. The mixture was allowed to reach room temperature and was stirred for $2 \mathrm{~h}$. The solution was filtrated over Celite and the solvent was removed under vacuum affording $5.91 \mathrm{~g}(95.3 \%)$ of the product as yellow oil. The product was used for the next step without further purification. HRMS (ESI): $[M+H]^{+}$, found 333.1281. $\mathrm{C}_{18} \mathrm{H}_{22} \mathrm{CIN}_{2} \mathrm{P}$ requires 333.1287. ${ }^{1} \mathrm{H}$ NMR $\left(\delta, \mathrm{CDCl}_{3}, 20^{\circ} \mathrm{C}\right): 7.41-7.30(\mathrm{~m}, 10 \mathrm{H}, \mathrm{Ph}), 4.30(\mathrm{br}, 2 \mathrm{H}, \mathrm{CH}), 3.12(\mathrm{~d}, \mathrm{~J}=$ $\left.3.0 \mathrm{~Hz}, \mathrm{CH}_{2}\right), 3.10\left(\mathrm{~d}, \mathrm{~J}=3.0 \mathrm{~Hz}, \mathrm{CH}_{2}\right), 1.76\left(\mathrm{~d}, \mathrm{~J}=3.0 \mathrm{~Hz}, \mathrm{CH}_{3}\right), 1.74\left(\mathrm{~d}, \mathrm{~J}=3.0 \mathrm{~Hz}, \mathrm{CH}_{3}\right) .{ }^{13} \mathrm{C}\left\{{ }^{1} \mathrm{H}\right\}$ $\operatorname{NMR}\left(\delta, \mathrm{CDCl}_{3}, 20^{\circ} \mathrm{C}\right): 143.3(\mathrm{Ph}), 128.7(\mathrm{Ph}), 127.6(\mathrm{Ph}), 127.0(\mathrm{Ph}), 58.1(\mathrm{CH}), 48.9(\mathrm{CH}), 22.6$ $\left(\mathrm{CH}_{2}\right) .{ }^{31} \mathrm{P}\left\{{ }^{1} \mathrm{H}\right\} \operatorname{NMR}\left(\delta, \mathrm{CDCl}_{3}, 20^{\circ} \mathrm{C}\right): 167.4 .^{2}$

$\mathrm{N}, \mathrm{N}$--Bis(1,3-bis[(S)-1-phenylethyl]-1,3,2-diazaphospholidine)-2,6-diaminopyridine (PNPSPEA) (1j).<smiles>CC(c1ccccc1)N1CCN(C(C)C(C)N2CCN(C(C)c3ccccc3)P2Nc2cccc(NP3N(C(C)c4ccccc4)CCN3C(C)c3ccccc3)n2)C1c1ccccc1</smiles> 
A solution of $n$-BuLi $(2.4 \mathrm{ml}, 6.0 \mathrm{mmol}$ ) was slowly added to a suspension of 2,6-diaminopyridine (330 $\mathrm{mg}, 3.0 \mathrm{mmol})$ in THF $(10 \mathrm{~mL})$. The solution was allowed to reach room temperature and was stirred for $2 \mathrm{~h}$. A solution of SPEA-PCI $(2.05 \mathrm{~g}, 6.0 \mathrm{mmol})$ in THF $(10 \mathrm{~mL})$ was added dropwise via syringe and the mixture was stirred for $2 \mathrm{~h}$. The solvent was removed under reduced pressure and the remaining solid was suspended in toluene and filtered over Celite. After removal of the solvent under reduced pressure PNP-SEPA was obtained as a sticky solid which was used without further purification. Yield: $2.0 \mathrm{~g}(95 \%)$. HRMS (ESI): $[\mathrm{M}+\mathrm{H}]^{+}$, found 702.3600. $\mathrm{C}_{41} \mathrm{H}_{49} \mathrm{~N}_{7} \mathrm{P}_{2}$ requires 702.3603 . Yield $2.0 \mathrm{~g}(95 \%) .{ }^{1} \mathrm{H}$ NMR $\left(\delta, \mathrm{CDCl}_{3}, 20^{\circ} \mathrm{C}\right): 7.43-7.26(\mathrm{~m}, 20 \mathrm{H}, \mathrm{Ph}), 7.17(\mathrm{t}, \mathrm{J}=9.9 \mathrm{~Hz}, \mathrm{py}), 8.81(\mathrm{~d}$, $\mathrm{J}=7.8 \mathrm{~Hz}, \mathrm{py}), 5.10(\mathrm{br}, 2 \mathrm{H}, \mathrm{NH}), 4.50(\mathrm{dd}, \mathrm{J}=13.3 \mathrm{~Hz}, 6.8 \mathrm{~Hz}, 4 \mathrm{H}, \mathrm{CH}), 3.10-2.82\left(\mathrm{~m}, 8 \mathrm{H}, \mathrm{CH}_{2}\right)$, $1.66\left(\mathrm{~d}, \mathrm{~J}=6.9 \mathrm{~Hz}, 12 \mathrm{H}, \mathrm{CH}_{3}\right) .{ }^{13} \mathrm{C}\left\{{ }^{1} \mathrm{H}\right\} \operatorname{NMR}\left(\delta, \mathrm{CDCl}_{3}, 20^{\circ} \mathrm{C}\right): 157.9\left(\mathrm{py}^{2,6}\right), 142.4(\mathrm{~d}, \mathrm{~J}=3.5 \mathrm{~Hz}, \mathrm{Ph})$, $142.2(\mathrm{~d}, \mathrm{~J}=5.5 \mathrm{~Hz}, \mathrm{Ph}), 128.6(\mathrm{Ph}), 128.5(\mathrm{Ph}), 127.4(\mathrm{Ph}), 127.1(\mathrm{Ph}), 127.0(\mathrm{Ph}), 97.3(\mathrm{py}), 54.7$ (d, J = 6.0 Hz, CH), $54.3\left(\mathrm{~d}, \mathrm{~J}=6.0 \mathrm{~Hz}, \mathrm{CH}\right.$ ), $42.9\left(\mathrm{~d}, \mathrm{~J}=8.0 \mathrm{~Hz}, \mathrm{CH}_{2}\right), 42.4\left(\mathrm{~d}, \mathrm{~J}=9.5 \mathrm{~Hz}, \mathrm{CH}_{2}\right), 20.7$ $\left(\mathrm{d}, \mathrm{J}=4.0 \mathrm{~Hz}, \mathrm{CH}_{3}\right), 20.4\left(\mathrm{~d}, \mathrm{~J}=4.0 \mathrm{~Hz}, \mathrm{CH}_{3}\right) \cdot{ }^{31} \mathrm{P}\left\{{ }^{1} \mathrm{H}\right\} \operatorname{NMR}\left(\delta, \mathrm{CDCl}_{3}, 20^{\circ} \mathrm{C}\right): 12.3$.

[Cr(PNP-Et)(CO) ${ }_{3}$ ] (2a). The product was obtained as red crystals in $89 \%$ yield. Anal. Calcd. for $\mathrm{C}_{16} \mathrm{H}_{25} \mathrm{CrN}_{3} \mathrm{O}_{3} \mathrm{P}_{2}$ (421.34): C, 45.61; $\mathrm{H}, 5.98 ; \mathrm{N}, 9.97$. Found: C, 45.71; H, 5.90; N, 9.88. ${ }^{1} \mathrm{H}$ NMR $\left(\delta\right.$, acetone- $\left.\mathrm{d}_{6}, 2{ }^{\circ} \mathrm{C}\right): 7.28(\mathrm{br}, 2 \mathrm{H}, \mathrm{NH}), 7.10\left(\mathrm{br}, 1 \mathrm{H}, \mathrm{py}^{4}\right), 6.11\left(\mathrm{br}, 2 \mathrm{H}, \mathrm{py}{ }^{3,5}\right), 2.23-2.18(\mathrm{~m}, 4 \mathrm{H}$, $\left.\mathrm{CH}_{2}\right), 2.23-2.05\left(\mathrm{~m}, 4 \mathrm{H}, \mathrm{CH}_{2}\right), 1.27-1.22\left(\mathrm{~m}, 12 \mathrm{H}, \mathrm{CH}_{3}\right) .{ }^{13} \mathrm{C}\left\{{ }^{1} \mathrm{H}\right\}$ NMR $\left(\delta\right.$, acetone- $\left.\mathrm{d}_{6}, 20^{\circ} \mathrm{C}\right): 235.7(\mathrm{t}, \mathrm{J}$ $=8.2 \mathrm{~Hz}, \mathrm{CO}), 223.8(\mathrm{t}, \mathrm{J}=17.1 \mathrm{~Hz}, \mathrm{CO}), 162.1\left(\mathrm{vt}, \mathrm{J}=10.6 \mathrm{~Hz}, \mathrm{py}^{2,6}\right), 137.7\left(\mathrm{py}^{4}\right), 97.6\left(\mathrm{py}^{3,5}\right), 27.3$ (vt, J = $\left.10.2 \mathrm{~Hz}, \mathrm{CH}_{2}\right), 27.1$ (vt, J = $\left.10.2 \mathrm{~Hz}, \mathrm{CH}_{2}\right), 8.2\left(\mathrm{CH}_{3}\right) .{ }^{31} \mathrm{P}\left\{{ }^{1} \mathrm{H}\right\}$ NMR ( $\delta$, acetone- $\mathrm{d}_{6}, 20{ }^{\circ} \mathrm{C}$ ): 130.0. IR (ATR, $\left.\mathrm{cm}^{-1}\right): 1905$ ( $\left.v_{\mathrm{CO}}\right), 1821\left(v_{\mathrm{CO}}\right), 1763\left(v_{\mathrm{CO}}\right)$.

[Cr(PNP-iPr)(CO) $)_{3}$ (2b). The product was obtained as yellow needles in $94 \%$ yield. Anal. Calcd. for $\mathrm{C}_{20} \mathrm{H}_{33} \mathrm{CrN}_{3} \mathrm{O}_{3} \mathrm{P}_{2} \cdot \mathrm{CH}_{3} \mathrm{CN}$ (518.50): C, 50.96; $\mathrm{H}, 7.00 ; \mathrm{N}, 10.81$. Found: C, 50.86; $\mathrm{H}, 6.93 ; \mathrm{N}$, 10.92. ${ }^{1} \mathrm{H}$ NMR $\left(\delta\right.$, acetone- $\left.\mathrm{d}_{6}, 20^{\circ} \mathrm{C}\right): 7.11\left(\mathrm{t}, \mathrm{J}=7.7 \mathrm{~Hz}, 3 \mathrm{H}, \mathrm{NH}, \mathrm{py}{ }^{4}\right), 6.32\left(\mathrm{br}, 2 \mathrm{H}, \mathrm{py}{ }^{3,5}\right), 2.35(\mathrm{bs}$, $4 \mathrm{H}, \mathrm{CH}), 1.35\left(\mathrm{~m}, 24 \mathrm{H}, \mathrm{CH}_{3}\right) \cdot{ }^{13} \mathrm{C}\left\{{ }^{1} \mathrm{H}\right\}$ NMR $\left(\delta\right.$, acetone- $\left.\mathrm{d}_{6}, 20^{\circ} \mathrm{C}\right): 236.2(\mathrm{t}, \mathrm{J}=8.3 \mathrm{~Hz}, \mathrm{CO}), 225.6(\mathrm{t}, \mathrm{J}$ $=17.3 \mathrm{~Hz}, \mathrm{CO}), 162.6\left(\mathrm{vt}, \mathrm{J}=10.4 \mathrm{~Hz}, \mathrm{py}^{2,6}\right), 137.8\left(\mathrm{py}^{4}\right), 97.4\left(\mathrm{vt}, \mathrm{J}=4.3 \mathrm{~Hz}, \mathrm{py} \mathrm{y}^{3,5}\right), 32.3(\mathrm{vt}, \mathrm{J}=9.4$ $\mathrm{Hz}, \mathrm{CH}$ ), 32.0 (vt, J = 9.4 Hz, CH), $19.1\left(\mathrm{vt}, \mathrm{J}=2.1 \mathrm{~Hz}, \mathrm{CH}_{3}\right), 18.5\left(\mathrm{vt}, \mathrm{J}=2.1 \mathrm{~Hz}, \mathrm{CH}_{3}\right) .{ }^{31} \mathrm{P}\left\{{ }^{1} \mathrm{H}\right\} \mathrm{NMR}$ $\left(\delta\right.$, acetone- $\left.\mathrm{d}_{6}, 20^{\circ} \mathrm{C}\right): 146.7$. IR $\left(\right.$ ATR, $\left.\mathrm{cm}^{-1}\right): 1923\left(v_{\mathrm{CO}}\right), 1785\left(v_{\mathrm{CO}}\right) .^{3}$

[Cr(PNP-Cy)(CO) $)_{3}$ (2c). The product was obtained as a yellow solid in $81 \%$ yield. Anal. Calcd. for $\mathrm{C}_{32} \mathrm{H}_{49} \mathrm{CrN}_{3} \mathrm{O}_{3} \mathrm{P}_{2} \cdot \mathrm{CH}_{3} \mathrm{CN}$ (637.71): C, 60.27; $\mathrm{H}, 7.75 ; \mathrm{N}, 6.59$. Found: $\mathrm{C}, 60.10 ; \mathrm{H}, 7.68 ; \mathrm{N}$, 6.67. ${ }^{1} \mathrm{H}$ NMR $\left(\delta\right.$, acetone- $\left.\mathrm{d}_{6}, 2 \mathrm{O}^{\circ} \mathrm{C}\right): 7.09\left(\mathrm{br}, 3 \mathrm{H}, \mathrm{NH}, \mathrm{py}{ }^{4}\right), 6.18\left(\mathrm{br}, 2 \mathrm{H}, \mathrm{py}{ }^{3,5}\right), 2.72-2.60(\mathrm{~m}, 4 \mathrm{H}, \mathrm{Cy})$, 2.33-1.32 (m, 40H, Cy). ${ }^{13} \mathrm{C}\left\{{ }^{1} \mathrm{H}\right\}$ NMR $\left(\delta\right.$, acetone- $\left.\mathrm{d}_{6}, 2 \mathrm{o}^{\circ} \mathrm{C}\right): 236.1(\mathrm{t}, \mathrm{J}=8.0 \mathrm{~Hz}, \mathrm{CO}), 225.8(\mathrm{t}, \mathrm{J}=$ 16.7 Hz, CO), 162.4 (vt, J = 8.7 Hz, py $\left.{ }^{2,6}\right), 137.7\left(\mathrm{py}^{4}\right), 97.4\left(\mathrm{py}^{3,5}\right), 43.4(\mathrm{vt}, \mathrm{J}=9.4 \mathrm{~Hz}, \mathrm{Cy}), 27.3$ (vt, J $=5.1 \mathrm{~Hz}, \mathrm{Cy}), 26.9$ (vt, J = 5.1 Hz, Cy), 26.1(Cy). ${ }^{31} \mathrm{P}\left\{{ }^{1} \mathrm{H}\right\}$ NMR ( $\delta$, acetone- $\left.\mathrm{d}_{6}, 20{ }^{\circ} \mathrm{C}\right): 149.8$. IR (ATR, cm-1): 1913 ( $\left.v_{\mathrm{CO}}\right), 1807$ ( $\left.v_{\mathrm{CO}}\right), 1793$ ( $\left.v_{\mathrm{CO}}\right)$.

[Cr(PNP-tBu)(CO) $\left.)_{3}\right](\mathbf{2 d})$. The reaction was performed for $5 \mathrm{~h}$ and the product was obtained as yellow needles in $78 \%$ yield. Anal. Calcd. for $\mathrm{C}_{24} \mathrm{H}_{41} \mathrm{CrN}_{3} \mathrm{O}_{3} \mathrm{P}_{2} \cdot \mathrm{CH}_{3} \mathrm{CN}$ (574.6). C, 54.35; $\mathrm{H}, 7.72 ; \mathrm{N}$, 9.75. Found: C, 54.11; H, 7.63; N, 9.80. ${ }^{1} \mathrm{H}$ NMR ( $\delta$, acetone- $\left.\mathrm{d}_{6}, 20{ }^{\circ} \mathrm{C}\right): 7.14\left(\mathrm{t}, \mathrm{J}=7.6 \mathrm{~Hz}, 1 \mathrm{H}, \mathrm{py}^{4}\right)$, 7.05 (bs, 2H, NH), 6.19 (d, J = 6.7 Hz, 2H, py $\left.\left.{ }^{3,5}\right), 1.43\left(\mathrm{~d}, \mathrm{~J}=12.5 \mathrm{~Hz}, 36 \mathrm{H}, \mathrm{CH}_{3}\right) .{ }^{13} \mathrm{C}^{1} \mathrm{H}\right\} \mathrm{NMR}(\delta$, acetone- $\left.\mathrm{d}_{6}, 20^{\circ} \mathrm{C}\right): 238.7(\mathrm{t}, \mathrm{J}=8.3 \mathrm{~Hz}, \mathrm{CO}), 233.0(\mathrm{t}, \mathrm{J}=15.6 \mathrm{~Hz}, \mathrm{CO}), 162.8\left(\mathrm{vt}, \mathrm{J}=8.3 \mathrm{~Hz}, \mathrm{py}^{2,6}\right)$, 
$138.7\left(\mathrm{py}^{4}\right), 98.0\left(\mathrm{py}^{3.5}\right), 41.3\left(\mathrm{vt}, \mathrm{J}=4.9 \mathrm{~Hz}, \mathrm{CH}_{3}, 29.5\left(\mathrm{CH}_{3}\right) .{ }^{31} \mathrm{P}\left\{{ }^{1} \mathrm{H}\right\}\right.$ NMR ( $\delta$, acetone- $\left.\mathrm{d}_{6}, 20{ }^{\circ} \mathrm{C}\right)$ : 164.4. IR (ATR, $\left.\mathrm{cm}^{-1}\right): 1908\left(v_{\mathrm{CO}}\right), 1792\left(v_{\mathrm{CO}}\right), 1756\left(v_{\mathrm{CO}}\right)$.

[Cr(PNP-Ph)(CO) $)_{3}$ (2e). The product was obtained as a yellow solid in $96 \%$ yield. Anal. Calcd. for $\mathrm{C}_{32} \mathrm{H}_{25} \mathrm{CrN}_{3} \mathrm{O}_{3} \mathrm{P}_{2}$ (613.51). C, 62.65; $\mathrm{H}, 4.11 ; \mathrm{N}, 6.85$. Found: C, 62.69; $\mathrm{H}, 4.04 ; \mathrm{N}, 6.78 .{ }^{1} \mathrm{H}$ $\operatorname{NMR}\left(\delta\right.$, acetone- $\left.\mathrm{d}_{6}, 20^{\circ} \mathrm{C}\right): 8.47(\mathrm{~d}, \mathrm{~J}=4.3 \mathrm{~Hz}, 2 \mathrm{H}, \mathrm{NH}), 7.79-7.73(\mathrm{~m}, 8 \mathrm{H}, \mathrm{Ph}), 7.45-7.33(\mathrm{~m}, 13 \mathrm{H}$, $\left.\mathrm{Ph}, \mathrm{py}^{4}\right), 6.55\left(\mathrm{~d}, \mathrm{~J}=6.1 \mathrm{~Hz}, 2 \mathrm{H}, \mathrm{py}^{3,5}\right) .{ }^{13} \mathrm{C}\left\{{ }^{1} \mathrm{H}\right\} \operatorname{NMR}\left(\delta\right.$, acetone- $\left.\mathrm{d}_{6}, 2 \mathrm{o}^{\circ} \mathrm{C}\right): 234.6(\mathrm{t}, \mathrm{J}=8.4 \mathrm{~Hz}, \mathrm{CO})$, $220.6(\mathrm{t}, \mathrm{J}=14.9 \mathrm{~Hz}, \mathrm{CO}), 161.0\left(\mathrm{vt}, \mathrm{J}=5.3 \mathrm{~Hz}, \mathrm{py}^{2,6}\right), 141.7(\mathrm{Ph}), 141.1(\mathrm{Ph}), 137.2\left(\mathrm{py}^{4}\right), 130.1(\mathrm{vt}, \mathrm{J}$ $=6.2 \mathrm{~Hz}, \mathrm{Ph}), 129.0(\mathrm{Ph}), 127.9(\mathrm{vt}, \mathrm{J}=4.7 \mathrm{~Hz}, \mathrm{Ph}), 116.7(\mathrm{Ph}), 97.9\left(\mathrm{py}{ }^{3,5}\right) .{ }^{31} \mathrm{P}\left\{{ }^{1} \mathrm{H}\right\} \mathrm{NMR}(\delta$, acetone$\left.\mathrm{d}_{6}, 20^{\circ} \mathrm{C}\right): 125.7 . \mathrm{IR}\left(\mathrm{ATR}, \mathrm{cm}^{-1}\right): 1973\left(v_{\mathrm{CO}}\right), 1840\left(v_{\mathrm{CO}}\right), 1807\left(v_{\mathrm{CO}}\right) .^{5}$

$\left[\mathbf{C r}\left(\mathrm{PNP}^{\mathrm{Me}}{ }_{-i \mathrm{Pr}}\right)(\mathbf{C O})_{3}\right](\mathbf{2 f})$. The product was obtained as yellow needles in $93.3 \%$ yield. Anal. Calcd. for $\mathrm{C}_{22} \mathrm{H}_{37} \mathrm{CrN}_{3} \mathrm{O}_{3} \mathrm{P}_{2}$ (505.50). C, 52.27; $\mathrm{H}, 7.38 ; \mathrm{N}, 8.31$. Found: $\mathrm{C}, 52.31 ; \mathrm{H}, 7.29 ; \mathrm{N}, 8.38$. ${ }^{1} \mathrm{H}$ $\operatorname{NMR}\left(\delta\right.$, acetone- $\left.\mathrm{d}_{6}, 20^{\circ} \mathrm{C}\right): 7.45\left(\mathrm{t}, \mathrm{J}=7.9 \mathrm{~Hz}, 1 \mathrm{H}, \mathrm{py}^{4}\right), 6.11\left(\mathrm{~d}, \mathrm{~J}=8.4 \mathrm{~Hz}, 2 \mathrm{H}, \mathrm{py}{ }^{3,5}\right), 2.99(\mathrm{~s}, 6 \mathrm{H}$, $\left.\mathrm{NCH}_{3}\right), 2.72-2.60(\mathrm{~m}, 4 \mathrm{H}, \mathrm{CH}), 1.40\left(\mathrm{dd}, \mathrm{J}=6.9 \mathrm{~Hz}, 12 \mathrm{H}, \mathrm{CH}_{3}\right), 1.14\left(\mathrm{dd}, \mathrm{J}=7.0 \mathrm{~Hz}, 12 \mathrm{H}, \mathrm{CH}_{3}\right.$ ). ${ }^{13} \mathrm{C}\left\{{ }^{1} \mathrm{H}\right\}$ NMR ( $\delta$, acetone- $\left.\mathrm{d}_{6}, 20^{\circ} \mathrm{C}\right): 235.20(\mathrm{t}, \mathrm{J}=8.7 \mathrm{~Hz}, \mathrm{CO}), 226.0(\mathrm{t}, \mathrm{J}=17.6 \mathrm{~Hz}, \mathrm{CO}), 163.0(\mathrm{vt}, \mathrm{J}$ $\left.=9.8 \mathrm{~Hz}, \mathrm{py}^{2,6}\right), 137.9\left(\mathrm{py}^{4}\right), 96.3\left(\mathrm{py}^{3,5}\right), 33.9(\mathrm{vt}, \mathrm{J}=8.9 \mathrm{~Hz}, \mathrm{CH}), 18.8\left(\mathrm{vt}, \mathrm{J}=5.1 \mathrm{~Hz}, \mathrm{CH}_{3}\right), 18.6$ $\left(\mathrm{CH}_{3}\right) .{ }^{31} \mathrm{P}\left\{{ }^{1} \mathrm{H}\right\} \operatorname{NMR}\left(\delta\right.$, acetone-d $\left.\mathrm{d}_{6}, 20^{\circ} \mathrm{C}\right):$ 171.5. IR $\left(\mathrm{ATR}, \mathrm{cm}^{-1}\right): 1926\left(v_{\mathrm{CO}}\right), 1795\left(v_{\mathrm{CO}}\right), 1774\left(v_{\mathrm{CO}}\right)$.

$\left[\mathrm{Cr}\left(\mathrm{PNP}^{\mathrm{Me}}-\mathrm{Ph}\right)(\mathrm{CO})_{3}\right](\mathbf{2 g})$. The product was obtained as yellow needles in $92 \%$ yield. Anal. Calcd. for $\mathrm{C}_{34} \mathrm{H}_{29} \mathrm{CrN}_{3} \mathrm{O}_{3} \mathrm{P}_{2} \cdot \mathrm{CH}_{3} \mathrm{CN}$ (682.62). C, 63.34; $\mathrm{H}, 4.73 ; \mathrm{N}, 8.21$. Found: $\mathrm{C}, 63.21 ; \mathrm{H}, 4.82 ; \mathrm{N}$, 8.31. ${ }^{1} \mathrm{H}$ NMR $\left(\delta\right.$, acetone- $\left.\left.\mathrm{d}_{6}, 2 \mathrm{o}^{\circ} \mathrm{C}\right): 7.68(\mathrm{t}, \mathrm{J}=7.7 \mathrm{~Hz}, 1 \mathrm{H}, \mathrm{py})^{4}\right), 7.64-7.60(\mathrm{~m}, 8 \mathrm{H}, \mathrm{Ph}), 7.51-7.44(\mathrm{~m}$, $12 \mathrm{H}, \mathrm{Ph}), 6.49\left(\mathrm{~d}, \mathrm{~J}=8.1 \mathrm{~Hz}, 2 \mathrm{H}, \mathrm{py}{ }^{3,5}\right), 3.10\left(\mathrm{~s}, 6 \mathrm{H}, \mathrm{NCH}_{3}\right) \cdot{ }^{13} \mathrm{C}\left\{{ }^{1} \mathrm{H}\right\}$ NMR ( $\delta$, acetone- $\left.\mathrm{d}_{6}, 20{ }^{\circ} \mathrm{C}\right)$ : 223.4 (t, J = 7.5 Hz, CO), 220.8 (t, J = 16.2 Hz, CO), 163.2 (vt, J = 13.0 Hz, py, $\left.{ }^{2,6}\right), 139.0\left(p^{4}\right)^{4}, 138.8$ (d, J = 6.3 Hz, Ph), 138.3 (d, J = 6.3 Hz, Ph), 131.5 (vt, J = 6.2 Hz, Ph), 130.1 (Ph), 129.1 (vt, J = 4.6 $\mathrm{Hz}, \mathrm{Ph}), 99.3\left(\mathrm{vt}, \mathrm{J}=3.3 \mathrm{~Hz}, \mathrm{py}^{3,5}\right), 37.5\left(\mathrm{vt}, \mathrm{J}=2.5 \mathrm{~Hz}, \mathrm{NCH}_{3}\right) .{ }^{31} \mathrm{P}\left\{{ }^{1} \mathrm{H}\right\}$ NMR $\left(\delta\right.$, acetone- $\left.\mathrm{d}_{6}, 20{ }^{\circ} \mathrm{C}\right)$ : 152.1. IR (ATR, $\left.\mathrm{cm}^{-1}\right): 1954$ ( $\left.v_{\mathrm{CO}}\right), 1842\left(v_{\mathrm{CO}}\right), 1819\left(v_{\mathrm{CO}}\right)$.

$\left[\mathrm{Cr}\left(\mathrm{PNP}^{\mathrm{Ph}}-\mathrm{Et}\right)(\mathrm{CO})_{3}\right](\mathbf{2 h})$. The product was obtained as a yellow solid in $94 \%$ yield. Anal. Calcd. for $\mathrm{C}_{28} \mathrm{H}_{33} \mathrm{CrN}_{3} \mathrm{O}_{3} \mathrm{P}_{2}$ (573.53). C, 58.64; $\mathrm{H}, 5.80 ; \mathrm{N}, 7.33$. Found: $\mathrm{C}, 58.51 ; \mathrm{H}, 5.95 ; \mathrm{N}, 7.43 .{ }^{1} \mathrm{H}$ $\operatorname{NMR}\left(\delta, \mathrm{CD}_{2} \mathrm{Cl}_{2}, 20^{\circ} \mathrm{C}\right): 7.57-7.42(\mathrm{~m}, 6 \mathrm{H}, \mathrm{Ph}), 7.18(\mathrm{~d}, \mathrm{~J}=7.4 \mathrm{~Hz}, 4 \mathrm{H}, \mathrm{Ph}), 6.87(\mathrm{t}, \mathrm{J}=7.8 \mathrm{~Hz}, 1 \mathrm{H}$, py $\left.^{4}\right), 5.35$ (br, $\left.2 \mathrm{H}, \mathrm{py}^{3,5}\right), 2.15-2.02\left(\mathrm{~m}, 8 \mathrm{H}, \mathrm{CH}_{2}\right), 1.35-1.19\left(\mathrm{~m}, 12 \mathrm{H}, \mathrm{CH}_{3}\right) \cdot{ }^{13} \mathrm{C}\left\{{ }^{1} \mathrm{H}\right\} \mathrm{NMR}\left(\delta, \mathrm{CD}_{2} \mathrm{Cl}_{2}, 20\right.$ $\left.{ }^{\circ} \mathrm{C}\right): 234.8(\mathrm{t}, \mathrm{J}=11.5 \mathrm{~Hz}, \mathrm{CO}), 222.8(\mathrm{br}, \mathrm{CO}), 163.7\left(\mathrm{vt}, \mathrm{J}=12.3 \mathrm{~Hz}, \mathrm{py}^{2,6}\right.$ ), $140.3(\mathrm{Ph}), 136.5\left(\mathrm{py}^{4}\right)$, 130.6 (Ph), $130.3(\mathrm{Ph}), 128.1(\mathrm{Ph}), 99.7\left(\mathrm{py}^{3,5}\right), 27.8\left(\mathrm{vt}, \mathrm{J}=9.9 \mathrm{~Hz} \mathrm{CH}_{2}\right), 27.6\left(\mathrm{vt}, \mathrm{J}=9.9 \mathrm{~Hz}, \mathrm{CH}_{2}\right.$ ), 8.6 $\left(\mathrm{CH}_{3}\right) .{ }^{31} \mathrm{P}\left\{{ }^{1} \mathrm{H}\right\} \operatorname{NMR}\left(\delta, \mathrm{CD}_{2} \mathrm{Cl}_{2}, 20^{\circ} \mathrm{C}\right)$ : 147.8. IR $\left(\mathrm{ATR}, \mathrm{cm}^{-1}\right): 1945\left(v_{\mathrm{CO}}\right), 1807\left(v_{\mathrm{CO}}\right)$.

[Cr(PNP-BIPOL)(CO) $)_{3}$ (2i). The reaction was performed for $5 \mathrm{~h}$ and the product was obtained as a yellow solid in $98 \%$ yield. Anal. Calcd. for $\mathrm{C}_{32} \mathrm{H}_{21} \mathrm{WN}_{3} \mathrm{O}_{7} \mathrm{P}_{2}$ (673.48). C, 57.07; $\mathrm{H}, 3.14 ; \mathrm{N}, 6.24$. Found: $\mathrm{C}, 57.13 ; \mathrm{H}, 3.05 ; \mathrm{N}, 6.31 .{ }^{1} \mathrm{H}$ NMR $\left(\delta\right.$, acetone- $\left.\mathrm{d}_{6}, 2 \mathrm{o}^{\circ} \mathrm{C}\right): 8.97(\mathrm{t}, \mathrm{J}=3.3 \mathrm{~Hz}, 2 \mathrm{H}, \mathrm{NH}), 7.70-$ $7.66(\mathrm{~m}, 4 \mathrm{H}, \mathrm{Ph}), 7.56-7.48\left(\mathrm{~m}, 5 \mathrm{H}, \mathrm{Ph}, \mathrm{py}^{4}\right), 7.44-7.39(\mathrm{~m}, 8 \mathrm{H}, \mathrm{Ph}), 6.56\left(\mathrm{~d}, \mathrm{~J}=8 \mathrm{~Hz}, 2 \mathrm{H}, \mathrm{py} \mathrm{y}^{3,5}\right)$. ${ }^{13} \mathrm{C}\left\{{ }^{1} \mathrm{H}\right\}$ NMR ( $\delta$, acetone- $\left.\mathrm{d}_{6}, 20{ }^{\circ} \mathrm{C}\right): 230.2(\mathrm{t}, \mathrm{J}=12.8 \mathrm{~Hz}, \mathrm{CO}), 217.9(\mathrm{t}, \mathrm{J}=22.1 \mathrm{~Hz}, \mathrm{CO}), 160.3(\mathrm{vt}, \mathrm{J}$ = $\left.14.0 \mathrm{~Hz}, \mathrm{py}^{2,6}\right), 150.9(\mathrm{vt}, \mathrm{J}=5.1 \mathrm{~Hz}, \mathrm{Ph}), 139.3\left(\mathrm{py}^{4}\right), 131.4(\mathrm{Ph}), 130.5(2 \mathrm{Ph}), 126.6(\mathrm{Ph}), 123.2$ $(\mathrm{Ph}), 100.3\left(\mathrm{py}^{3,5}\right) .{ }^{31} \mathrm{P}\left\{{ }^{1} \mathrm{H}\right\}$ NMR $\left(\delta\right.$, acetone- $\left.\mathrm{d}_{6}, 20^{\circ} \mathrm{C}\right): 230.6$. IR (ATR, $\left.\mathrm{cm}^{-1}\right): 1972\left(v_{\mathrm{CO}}\right), 1860\left(v_{\mathrm{CO}}\right)$.

[Cr(PNP-SPEA)(CO) $\left.)_{3}\right](2 \mathrm{j})$. The reaction was performed for $3 \mathrm{~h}$ and the product was obtained as a yellow solid in 93\% yield. Anal. Calcd. for $\mathrm{C}_{44} \mathrm{H}_{49} \mathrm{CrN}_{7} \mathrm{O}_{3} \mathrm{P}_{2}$ (837.87). C, 63.08; $\mathrm{H}, 5.89 ; \mathrm{N}, 11.70$. 
Found: $\mathrm{C}, 63.15 ; \mathrm{H}, 5.91 ; \mathrm{N}, 11.63 .{ }^{1} \mathrm{H}$ NMR $\left(\delta, \mathrm{CD}_{2} \mathrm{Cl}_{2}, 20^{\circ} \mathrm{C}\right): 7.56(\mathrm{~d}, \mathrm{~J}=7.6 \mathrm{~Hz}, 4 \mathrm{H}, \mathrm{Ph}), 7.38-7.20$ (m, 16H, Ph), 5.96 (bs, 2H), 5.47 (bs, 3H), 3.19-2.77 (m, 12H, CH, $\mathrm{CH}), 2.77$ (bs, 2H), 1.67 (s, 6H, $\left.\mathrm{CH}_{3}\right), 1.53\left(\mathrm{~s}, 6 \mathrm{H}, \mathrm{CH}_{3}\right) .{ }^{13} \mathrm{C}\left\{{ }^{1} \mathrm{H}\right\} \operatorname{NMR}\left(\delta, \mathrm{CD}_{2} \mathrm{Cl}_{2}, 20^{\circ} \mathrm{C}\right): 236.2(\mathrm{CO}), 223.9(\mathrm{CO}), 158.7-158.5\left(\mathrm{py}^{2,6}\right)$, $143.5(\mathrm{Ph}), 143,0$ (t, J = 5.6 Hz, Ph), $136.8\left(\mathrm{py}^{4}\right), 128.2(\mathrm{Ph}), 128.0(\mathrm{Ph}), 127.4(\mathrm{Ph}), 127.3(\mathrm{Ph}), 126.7$ (Ph), $126.4(\mathrm{Ph}), 97.0\left(\mathrm{py}^{3,5}\right), 52.1(\mathrm{t}, \mathrm{J}=6.0 \mathrm{~Hz}, \mathrm{CH}), 50.2(\mathrm{t}, \mathrm{J}=5.4 \mathrm{~Hz}, \mathrm{CH}), 41.3\left(\mathrm{CH}_{2}\right), 41.1\left(\mathrm{CH}_{2}\right)$, $17.6\left(\mathrm{CH}_{3}\right), 17.4\left(\mathrm{CH}_{3}\right) \cdot{ }^{31} \mathrm{P}\left\{{ }^{1} \mathrm{H}\right\} \operatorname{NMR}\left(\delta, \mathrm{CD}_{2} \mathrm{Cl}_{2}, 20^{\circ} \mathrm{C}\right): 193.5 . \mathrm{IR}\left(\mathrm{ATR}, \mathrm{cm}^{-1}\right): 1936.3\left(v_{\mathrm{CO}}\right), 1827$ $\left(v_{\mathrm{CO}}\right), 1811\left(v_{\mathrm{CO}}\right)$.

[Mo(PNP-Et)(CO) $)_{3}$ (3a). The product was obtained as yellow crystals in $91 \%$ yield. Anal. Calcd. for $\mathrm{C}_{16} \mathrm{H}_{25} \mathrm{MoN}_{3} \mathrm{O}_{3} \mathrm{P}_{2}$ (465.30). C, 41.30; $\mathrm{H}, 5.42 ; \mathrm{N}, 9.03$. Found: $\mathrm{C}, 41.21 ; \mathrm{H}, 5.55 ; \mathrm{N}, 9.11 .{ }^{1} \mathrm{H}$ $\operatorname{NMR}\left(\delta, \mathrm{CD}_{2} \mathrm{Cl}_{2}, 20^{\circ} \mathrm{C}\right): 7.12\left(\mathrm{t}, \mathrm{J}=7.9 \mathrm{~Hz}, 1 \mathrm{H}, \mathrm{py}^{4}\right), 6.07\left(\mathrm{~d}, \mathrm{~J}=8.0 \mathrm{~Hz}, 2 \mathrm{H}, \mathrm{py} \mathrm{y}^{3,5}\right), 5,32(\mathrm{~s}, 2 \mathrm{H}, \mathrm{NH})$, 2.11-2.06 (m, 4H, CH $), 2.03-1.97\left(\mathrm{~m}, 4 \mathrm{H}, \mathrm{CH}_{2}\right), 1.26-1.13\left(\mathrm{~m}, 12 \mathrm{H}, \mathrm{CH}_{3}\right) \cdot{ }^{13} \mathrm{C}\left\{{ }^{1} \mathrm{H}\right\} \mathrm{NMR}\left(\delta, \mathrm{CD}_{2} \mathrm{Cl}_{2}, 20\right.$ $\left.{ }^{\circ} \mathrm{C}\right): 230.2(\mathrm{t}, \mathrm{J}=5.2 \mathrm{~Hz}, \mathrm{CO}), 213.8(\mathrm{t}, \mathrm{J}=10.5 \mathrm{~Hz}, \mathrm{CO}), 160.2\left(\mathrm{vt}, \mathrm{J}=8.6 \mathrm{~Hz}, \mathrm{py}^{2,6}\right), 137.1\left(\mathrm{py}^{4}\right), 97.4$ (vt, J = 2,8 Hz, py $\left.{ }^{3,5}\right), 28.1\left(\mathrm{vt}, \mathrm{J}=10.8 \mathrm{~Hz}, \mathrm{CH}_{2}\right), 7.8\left(\mathrm{CH}_{3}\right) \cdot{ }^{31} \mathrm{P}\left\{{ }^{1} \mathrm{H}\right\} \operatorname{NMR}\left(\delta, \mathrm{CD}_{2} \mathrm{Cl}_{2}, 20{ }^{\circ} \mathrm{C}\right): 111.3 . \mathrm{IR}$ $\left(\right.$ ATR, $\left.\mathrm{cm}^{-1}\right): 1929\left(v_{\mathrm{CO}}\right), 1840\left(v_{\mathrm{CO}}\right), 1780\left(v_{\mathrm{CO}}\right)$.

[Mo(PNP-iPr)(CO) $)_{3}$ (3b). The product was obtained as a yellow solid in $93 \%$ yield. The identity of this complex was confirmed by comparison with an authentic sample prepared recently by a different method. ${ }^{4}$

[Mo(PNP-Cy)(CO) $)_{3}$ (3c). The product was obtained as a yellow solid in $96 \%$ yield. Anal. Calcd. for $\mathrm{C}_{32} \mathrm{H}_{49} \mathrm{MoN}_{3} \mathrm{O}_{3} \mathrm{P}_{2}$ (681.67). C, 56.38; $\mathrm{H}, 7.25 ; \mathrm{N}, 6.15$. Found: $\mathrm{C}, 56.21 ; \mathrm{H}, 7.35 ; \mathrm{N}, 6.31 .{ }^{1} \mathrm{H}$ $\operatorname{NMR}\left(\delta, \mathrm{CD}_{2} \mathrm{Cl}_{2}, 20^{\circ} \mathrm{C}\right): 7.16\left(\mathrm{t}, \mathrm{J}=7.6 \mathrm{~Hz}, 1 \mathrm{H}, \mathrm{py}^{4}\right), 6.11\left(\mathrm{~d}, \mathrm{~J}=7.8 \mathrm{~Hz}, 2 \mathrm{H}, \mathrm{py}{ }^{3,5}\right), 5.32(\mathrm{~s}, 2 \mathrm{H}, \mathrm{NH})$, 2.14-1.77 (m, 22H, Cy), 1.63-1.19 (m, 22H, Cy). ${ }^{13} \mathrm{C}\left\{{ }^{1} \mathrm{H}\right\} \mathrm{NMR}\left(\delta, \mathrm{CD}_{2} \mathrm{Cl}_{2}, 20{ }^{\circ} \mathrm{C}\right): 231.1$ (br, CO), 216.4 (t, J = $10.0 \mathrm{~Hz}, \mathrm{CO}), 160.9\left(\mathrm{vt}, \mathrm{J}=7.0 \mathrm{~Hz}, \mathrm{py}^{2,6}\right), 137.6\left(\mathrm{py}^{4}\right), 97.5\left(\mathrm{py}^{3,5}\right), 42.9(\mathrm{vt}, \mathrm{J}=8.9 \mathrm{~Hz}, \mathrm{Cy})$, $28.8(\mathrm{~d}, \mathrm{~J}=32.3 \mathrm{~Hz}, \mathrm{Cy}), 27.5$ (d, J = 25.6 Hz, Cy), 26.7 (Cy). ${ }^{31} \mathrm{P}\left\{{ }^{1} \mathrm{H}\right\} \mathrm{NMR}\left(\delta, \mathrm{CD}_{2} \mathrm{Cl}_{2}, 20{ }^{\circ} \mathrm{C}\right): 122.6$. IR (ATR, cm $\left.{ }^{-1}\right)$ : $1941\left(v_{\mathrm{co}}\right), 1828\left(v_{\mathrm{co}}\right), 1790\left(v_{\mathrm{CO}}\right)$.

$\left.\left[\mathrm{Mo}(\mathrm{PNP}-\mathrm{tBu})(\mathbf{C O})_{3}\right] \mathbf{( 3 d}\right)$. The reaction was performed at $155^{\circ} \mathrm{C}$ and the product was obtained as a yellow solid in $78 \%$ yield. The identity of this complex was confirmed by comparison with an authentic sample prepared recently by a different method. ${ }^{4}$

[Mo(PNP-Ph)(CO) $)_{3}$ (3e). The product was obtained as a yellow solid in $92 \%$ yield. The identity of this complex was confirmed by comparison with an authentic sample prepared recently by a different method. ${ }^{3,4}$

$\left[\mathrm{Mo}\left(\mathrm{PNP}^{\mathrm{Me}}-\mathrm{Pr}\right)(\mathbf{C O})_{3}\right]$ (3f). The product was obtained as a yellow solid in $86 \%$ yield. The identity of this complex was confirmed by comparison with an authentic sample prepared recently by a different method. ${ }^{5}$

$\left[\mathrm{Mo}\left(\mathrm{PNP}^{\mathrm{Me}}-\mathrm{Ph}\right)(\mathbf{C O})_{3}\right](\mathbf{3 g})$. The reaction was performed for $5 \mathrm{~h}$ and the product was obtained as yellow crystals in $92 \%$ yield. The identity of this complex was confirmed by comparison with an authentic sample prepared recently by a different method $^{6}$

$\left[\mathrm{Mo}\left(\mathrm{PNP}^{\mathrm{Ph}}-\mathrm{Et}\right)(\mathrm{CO})_{3}\right]$ (3h). The product was obtained as a yellow-brown solid in $99 \%$ yield. Anal. Calcd. for $\mathrm{C}_{28} \mathrm{H}_{33} \mathrm{MoN}_{3} \mathrm{O}_{3} \mathrm{P}_{2}$ (617.50). C, 54.46; $\mathrm{H}, 5.39 ; \mathrm{N}, 6.81$. Found: C, 54.50; H, 5.35; N, 6.88. ${ }^{1} \mathrm{H}$ NMR $\left(\delta, \mathrm{CD}_{2} \mathrm{Cl}_{2}, 20{ }^{\circ} \mathrm{C}\right): 7.54-7.38(\mathrm{~m}, 6 \mathrm{H}, \mathrm{Ph}), 7.27-7.12(\mathrm{~m}, 4 \mathrm{H}, \mathrm{Ph}), 6.89\left(\mathrm{br}, 1 \mathrm{H}, \mathrm{py}{ }^{4}\right), 5.35$ (br, 2H, py $\left.\left.{ }^{3,5}\right), 1.98\left(\mathrm{br}, 8 \mathrm{H}, \mathrm{CH}_{2}\right), 1.29\left(\mathrm{br}, 12 \mathrm{H}, \mathrm{CH}_{3}\right) \cdot{ }^{13} \mathrm{C}^{1}{ }^{1} \mathrm{H}\right\} \operatorname{NMR}\left(\delta, \mathrm{CD}_{2} \mathrm{Cl}_{2}, 20{ }^{\circ} \mathrm{C}\right): 229.9(\mathrm{t}, \mathrm{J}=$ 
5.0 Hz, CO), 214.3 (t, J = 10.5 Hz, CO), $163.5\left(v t, J=10.6 \mathrm{~Hz}, \mathrm{py}^{2,6}\right), 140.5(\mathrm{Ph}), 136.7\left(\mathrm{py}^{4}\right), 130.8$ (Ph), $130.4(\mathrm{Ph}), 128.3(\mathrm{Ph}), 100.8\left(\mathrm{py}^{3,5}\right), 28.4\left(\mathrm{vt}, \mathrm{J}=10.8 \mathrm{~Hz}, \mathrm{CH}_{2}\right) 28.2\left(\mathrm{vt}, \mathrm{J}=10.7 \mathrm{~Hz}, \mathrm{CH}_{2}\right), 9.1$ $\left(\mathrm{CH}_{3}\right) .{ }^{31} \mathrm{P}\left\{{ }^{1} \mathrm{H}\right\} \operatorname{NMR}\left(\delta, \mathrm{CD}_{2} \mathrm{Cl}_{2}, 20^{\circ} \mathrm{C}\right): 129.5$. IR $\left(\mathrm{ATR}, \mathrm{cm}^{-1}\right): 1949\left(v_{\mathrm{CO}}\right), 1815\left(v_{\mathrm{CO}}\right)$.

[Mo(PNP-BIPOL)(CO) $)_{3}$ (3i). The product was obtained as a yellow solid in $95 \%$ yield. Anal. Calcd. for $\mathrm{C}_{32} \mathrm{H}_{21} \mathrm{MoN}_{3} \mathrm{O}_{7} \mathrm{P}_{2}$ (717.44). C, 53.57; $\mathrm{H}, 2.95 ; \mathrm{N}, 5.86$. Found: $\mathrm{C}, 53.51 ; \mathrm{H}, 2.89 ; \mathrm{N}, 5.80 .{ }^{1} \mathrm{H}$ $\operatorname{NMR}\left(\delta\right.$, acetone- $\left.\mathrm{d}_{6}, 20^{\circ} \mathrm{C}\right): 8.96(\mathrm{~s}, 2 \mathrm{H}, \mathrm{NH}), 7.78(\mathrm{dd}, \mathrm{J}=7.7 \mathrm{~Hz}, \mathrm{~J}=1.7 \mathrm{~Hz}, 4 \mathrm{H}, \mathrm{Ph}), 7.55-7.48(\mathrm{~m}$, $\left.5 \mathrm{H}, \mathrm{Ph}, \mathrm{py}{ }^{4}\right), 7.44-7.37(\mathrm{~m}, 8 \mathrm{H}, \mathrm{Ph}), 6.62\left(\mathrm{~d}, \mathrm{~J}=8 \mathrm{~Hz}, 2 \mathrm{H}, \mathrm{py}{ }^{3,5}\right) .{ }^{13} \mathrm{C}\left\{{ }^{1} \mathrm{H}\right\}$ NMR $\left(\delta\right.$, acetone- $\left.\mathrm{d}_{6}, 20^{\circ} \mathrm{C}\right)$ : $224.7(\mathrm{t}, \mathrm{J}=8.5 \mathrm{~Hz}, \mathrm{CO}), 208.4(\mathrm{t}, \mathrm{J}=13.7 \mathrm{~Hz}, \mathrm{CO}), 159.4\left(\mathrm{vt}, \mathrm{J}=11.4 \mathrm{~Hz}, \mathrm{py}^{2,6}\right), 150.7(\mathrm{vt}, \mathrm{J}=4.5$ $\mathrm{Hz}, \mathrm{Ph}), 139.8\left(\mathrm{py}^{4}\right), 131.5(\mathrm{Ph}), 130.6(2 \mathrm{Ph}), 126.6(\mathrm{Ph}), 123.2(\mathrm{Ph}), 100.7\left(\mathrm{py}^{3,5}\right) .{ }^{31} \mathrm{P}\left\{{ }^{1} \mathrm{H}\right\} \mathrm{NMR}(\delta$, acetone- $\left.\mathrm{d}_{6}, 20^{\circ} \mathrm{C}\right): 204.8 . \mathrm{IR}\left(\mathrm{ATR}, \mathrm{cm}^{-1}\right): 1985\left(v_{\mathrm{CO}}\right), 1876\left(v_{\mathrm{CO}}\right)$.

[W(PNP-Et)(CO) $)_{3}$ (4a). The product was obtained as a yellow solid in $79 \%$ yield. Anal. Calcd. for $\mathrm{C}_{16} \mathrm{H}_{25} \mathrm{WN}_{3} \mathrm{O}_{3} \mathrm{P}_{2}$ (553.18). C, 34.74; $\mathrm{H}, 4.56 ; \mathrm{N}, 7.60$. Found: $\mathrm{C}, 34.61 ; \mathrm{H}, 4.65 ; \mathrm{N}, 7.55 .{ }^{1} \mathrm{H}$ NMR $(\delta$, $\left.\mathrm{CD}_{2} \mathrm{Cl}_{2}, 2 \mathrm{o}^{\circ} \mathrm{C}\right): 7.06\left(\mathrm{t}, \mathrm{J}=8.0 \mathrm{~Hz}, 1 \mathrm{H}, \mathrm{py}^{4}\right), 6.06\left(\mathrm{~d}, \mathrm{~J}=8.0 \mathrm{~Hz}, 2 \mathrm{H}, \mathrm{py}^{3,5}\right), 5,63(\mathrm{~s}, 2 \mathrm{H}, \mathrm{NH}), 2.18-2.11$ $\left(\mathrm{m}, 4 \mathrm{H}, \mathrm{CH}_{2}\right), 2.02-1.95\left(\mathrm{~m}, 4 \mathrm{H}, \mathrm{CH}_{2}\right), 1.15-1.07\left(\mathrm{~m}, 12 \mathrm{H}, \mathrm{CH}_{3}\right) \cdot{ }^{13} \mathrm{C}\left\{{ }^{1} \mathrm{H}\right\} \operatorname{NMR}\left(\delta, \mathrm{CD}_{2} \mathrm{Cl}_{2}, 20{ }^{\circ} \mathrm{C}\right): 222.0$ (br, CO), $208.2(\mathrm{t}, \mathrm{J}=7.5 \mathrm{~Hz}, \mathrm{CO}), 161.4\left(\mathrm{vt}, \mathrm{J}=8.7 \mathrm{~Hz}, \mathrm{py}^{2.6}\right), 137.0\left(\mathrm{py}^{4}\right), 97.1\left(\mathrm{vt}, \mathrm{J}=2,8 \mathrm{~Hz}, \mathrm{py} \mathrm{y}^{3,5}\right.$ ), $28.7\left(\mathrm{vt}, \mathrm{J}=13.4 \mathrm{~Hz}, \mathrm{CH}_{2}\right), 8.1\left(\mathrm{CH}_{3}\right) \cdot{ }^{31} \mathrm{P}\left\{{ }^{1} \mathrm{H}\right\} \operatorname{NMR}\left(\delta, \mathrm{CD}_{2} \mathrm{Cl}_{2}, 20{ }^{\circ} \mathrm{C}\right): 94.0\left({ }^{1} \mathrm{~J}_{\mathrm{w}-\mathrm{p}}=324.4 \mathrm{~Hz}\right)$. IR $\left(\right.$ ATR, $\left.\mathrm{cm}^{-1}\right): 1921\left(v_{\mathrm{CO}}\right), 1834\left(v_{\mathrm{CO}}\right), 1768\left(v_{\mathrm{CO}}\right)$.

[W(PNP-iPr)(CO) $\left.{ }_{3}\right]$ (4b). The product was obtained as yellow crystals in $96 \%$ yield. The identity of this complex was confirmed by comparison with an authentic sample prepared recently by a different method. ${ }^{3,5}$

[W(PNP-Cy)(CO) $)_{3}$ (4C). The reaction was performed at $155^{\circ} \mathrm{C}$ for $5 \mathrm{~h}$ and the product was obtained as yellow crystals in $84 \%$ yield. Anal. Calcd. for $\mathrm{C}_{32} \mathrm{H}_{49} \mathrm{WN}_{3} \mathrm{O}_{3} \mathrm{P}_{2}$ (769.55). C, 49.95; $\mathrm{H}, 6.42$; $\mathrm{N}, 5.46$. Found: $\mathrm{C}, 49.80 ; \mathrm{H}, 6.49 ; \mathrm{N}, 5.53 .{ }^{1} \mathrm{H} \operatorname{NMR}\left(\delta\right.$, acetone- $\left.\mathrm{d}_{6}, 2 \mathrm{o}^{\circ} \mathrm{C}\right): 7.40(\mathrm{~s}, 2 \mathrm{H}, \mathrm{NH}), 7.04(\mathrm{t}, \mathrm{J}$ $\left.=8.4 \mathrm{~Hz}, 1 \mathrm{H}, \mathrm{py}^{4}\right), 6.19\left(\mathrm{~d}, \mathrm{~J}=7.8 \mathrm{~Hz}, 2 \mathrm{H}, \mathrm{py}^{3,5}\right), 2.09-1.19(\mathrm{~m}, 44 \mathrm{H}, \mathrm{Cy}) .{ }^{13} \mathrm{C}\left\{{ }^{1} \mathrm{H}\right\}$ NMR $\left(\delta\right.$, acetone- $\mathrm{d}_{6}$, $20^{\circ} \mathrm{C}$ ): 222.3 (bt, CO), $211.3(\mathrm{t}, \mathrm{J}=7.3 \mathrm{~Hz}, \mathrm{CO}), 162.8\left(\mathrm{vt}, \mathrm{J}=8.5 \mathrm{~Hz}, \mathrm{py}^{2,6}\right), 137.3\left(\mathrm{py}^{4}\right), 96.9\left(\mathrm{py}^{3,5}\right)$, 43.5 (vt, J = 12.4 Hz, Cy), 27.3 (vt, J = 5.6 Hz, Cy), 26.9 (vt, J = 5.6 Hz, Cy), 26.4 (Cy). ${ }^{31} \mathrm{P}\left\{{ }^{1} \mathrm{H}\right\} \mathrm{NMR}$ $\left(\delta\right.$, acetone- $\left.\mathrm{d}_{6}, 20^{\circ} \mathrm{C}\right): 109.5\left({ }^{1} \mathrm{~J}_{\mathrm{W}-\mathrm{p}}=320.3 \mathrm{~Hz}\right)$. IR (ATR, $\left.\mathrm{cm}^{-1}\right): 1933\left(v_{\mathrm{CO}}\right), 1807\left(v_{\mathrm{CO}}\right), 1773\left(v_{\mathrm{CO}}\right)$.

[W(PNP-tBu)(CO) $)_{3}$ (4d). The reaction was performed at $155^{\circ} \mathrm{C}$ and product was obtained as yellow crystals in $85 \%$ yield. The identity of this complex was confirmed by comparison with an authentic sample prepared recently by a different method. ${ }^{5}$

[W(PNP-Ph)(CO) $)_{3}$ (4e). The product was obtained as yellow crystals in $93 \%$ yield. The identity of this complex was confirmed by comparison with an authentic sample prepared recently by a different method.1, ${ }^{3,5}$

[W(PNP $\left.\left.{ }^{\mathrm{Me}}-\mathrm{Pr}\right)(\mathrm{CO})_{3}\right](\mathbf{4 f})$. The product was obtained as yellow needles in $92 \%$ yield. Anal. Calcd. for $\mathrm{C}_{22} \mathrm{H}_{37} \mathrm{WN}_{3} \mathrm{O}_{3} \mathrm{P}_{2} \cdot \mathrm{CH}_{3} \mathrm{CN}$ (678.40). C, 42.49; $\mathrm{H}, 5.94 ; \mathrm{N}, 8.26$. Found: C, 42.54; $\mathrm{H}, 6.06 ; \mathrm{N}$, 8.23. ${ }^{1} \mathrm{H}$ NMR $\left(\delta, \mathrm{CD}_{2} \mathrm{Cl}_{2}, 2 \mathrm{o}^{\circ} \mathrm{C}\right): 7.44\left(\mathrm{t}, \mathrm{J}=6.9 \mathrm{~Hz}, 1 \mathrm{H}, \mathrm{py}^{4}\right), 6.07\left(\mathrm{~d}, \mathrm{~J}=8.0 \mathrm{~Hz}, 2 \mathrm{H}, \mathrm{py}{ }^{3,5}\right), 3.04(\mathrm{~s}$, $\left.6 \mathrm{H}, \mathrm{NCH}_{3}\right), 2.47-2.36(\mathrm{~m}, 4 \mathrm{H}, \mathrm{CH}), 1.40-1.30\left(\mathrm{~m}, 12 \mathrm{H}, \mathrm{CH}_{3}\right), 1.17-1.02\left(\mathrm{~m}, 12 \mathrm{H}, \mathrm{CH}_{3}\right) .{ }^{13} \mathrm{C}\left\{{ }^{1} \mathrm{H}\right\} \mathrm{NMR}$ $\left(\delta, \mathrm{CD}_{2} \mathrm{Cl}_{2}, 2{ }^{\circ} \mathrm{C}\right): 222.5(\mathrm{t}, \mathrm{J}=1.7 \mathrm{~Hz}, \mathrm{CO}), 211.6(\mathrm{t}, \mathrm{J}=7.8 \mathrm{~Hz}, \mathrm{CO}), 163.5\left(\mathrm{vt}, \mathrm{J}=9.3 \mathrm{~Hz}, \mathrm{py}^{2.6}\right.$ ), $137.7\left(\mathrm{py}^{4}\right), 96.9\left(\mathrm{py}^{3,5}\right), 34.2\left(\mathrm{NCH}_{3}\right), 33.8(\mathrm{vt}, \mathrm{J}=11.7 \mathrm{~Hz}, \mathrm{CH}), 19.6\left(\mathrm{vt}, \mathrm{J}=6.1 \mathrm{~Hz}, \mathrm{CH}_{3}\right), 18.3\left(\mathrm{CH}_{3}\right)$. 
${ }^{31} \mathrm{P}\left\{{ }^{1} \mathrm{H}\right\} \operatorname{NMR}\left(\delta, \mathrm{CD}_{2} \mathrm{Cl}_{2}, 20^{\circ} \mathrm{C}\right): 144.0\left({ }^{1} \mathrm{~J}_{\mathrm{w}-\mathrm{p}}=326.2 \mathrm{~Hz}\right)$. IR $\left(\mathrm{ATR}, \mathrm{cm}^{-1}\right): 1928\left(v_{\mathrm{CO}}\right), 1890\left(v_{\mathrm{CO}}\right), 1797$ $\left(v_{\mathrm{CO}}\right)$.

[W(PNP $\left.\left.{ }^{\mathrm{Me}}-\mathbf{P h}\right)(\mathbf{C O})_{3}\right] \mathbf{( 4 g ) . ~ T h e ~ p r o d u c t ~ o b t a i n e d ~ w e r e ~ y e l l o w ~ n e e d l e s ~ i n ~} 95 \%$ yield. Anal. Calcd. for $\mathrm{C}_{34} \mathrm{H}_{29} \mathrm{WN}_{3} \mathrm{O}_{3} \mathrm{P}_{2} \cdot \mathrm{CH}_{3} \mathrm{CN}$ (814.46). C, 53.09; $\mathrm{H}, 3.99 ; \mathrm{N}, 6.88$. Found: $\mathrm{C}, 53.15 ; \mathrm{H}, 4.05 ; \mathrm{N}$, 6.92. ${ }^{1} \mathrm{H}$ NMR $\left(\delta\right.$, acetone- $\left.\mathrm{d}_{6}, 2 \mathrm{O}^{\circ} \mathrm{C}\right): 7.71\left(\mathrm{t}, \mathrm{J}=8.4 \mathrm{~Hz}, 1 \mathrm{H}, \mathrm{py}{ }^{4}\right), 7.65-7.60(\mathrm{~m}, 8 \mathrm{H}, \mathrm{Ph}), 7.54-7.41(\mathrm{~m}$,

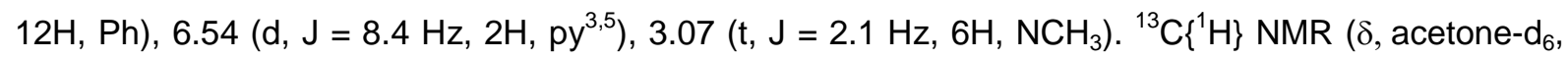
$\left.20^{\circ} \mathrm{C}\right): 221.1$ (br, CO), $207.4(\mathrm{t}, \mathrm{J}=7.0 \mathrm{~Hz}, \mathrm{CO}), 163.5\left(\mathrm{vt}, \mathrm{J}=11.3 \mathrm{~Hz}, \mathrm{py}^{2,6}\right), 139.1\left(\mathrm{py}^{4}\right), 138.9(\mathrm{Ph})$, 138.7 (Ph), 131.7 (vt, J = 6.4 Hz, Ph), 130.4 (s, Ph), 129.2 (vt, J = 4.9 Hz, Ph), 100.4 (vt, J = 3.3 Hz, $\left.\mathrm{py}^{3,5}\right), 38.1\left(\mathrm{vt}, \mathrm{J}=1.7 \mathrm{~Hz}, \mathrm{NCH}_{3}\right) \cdot{ }^{31} \mathrm{P}\left\{{ }^{1} \mathrm{H}\right\} \operatorname{NMR}\left(\delta\right.$, acetone- $\left.\mathrm{d}_{6}, 20^{\circ} \mathrm{C}\right): 114.7\left({ }^{1} \mathrm{~J}_{\mathrm{w}-\mathrm{p}}=342.1 \mathrm{~Hz}\right) . \mathrm{IR}$ $\left(\right.$ ATR, $\left.\mathrm{cm}^{-1}\right)$ : $1954\left(v_{\mathrm{CO}}\right), 1839\left(v_{\mathrm{CO}}\right), 1801\left(v_{\mathrm{CO}}\right)$.

$\left[\mathbf{W}\left(\mathrm{PNP}^{\mathrm{Ph}}-\mathrm{Et}\right)(\mathrm{CO})_{3}\right](\mathbf{4 h})$. The product obtained were yellow crystals in $94 \%$ yield. Anal. Calcd. for $\mathrm{C}_{28} \mathrm{H}_{33} \mathrm{WN}_{3} \mathrm{O}_{3} \mathrm{P}_{2}$ (705.38). C, 47.68; $\mathrm{H}, 4.72 ; \mathrm{N}, 5.96$. Found: $\mathrm{C}, 47.71 ; \mathrm{H}, 4.81 ; \mathrm{N}, 5.92 .{ }^{1} \mathrm{H}$ $\operatorname{NMR}\left(\delta, \mathrm{CD}_{2} \mathrm{Cl}_{2}, 20^{\circ} \mathrm{C}\right): 7.62-7.47(\mathrm{~m}, 6 \mathrm{H}, \mathrm{Ph}), 7.25-7.19(\mathrm{~m}, 4 \mathrm{H}, \mathrm{Ph}), 6.92(\mathrm{tt}, \mathrm{J}=8.3 \mathrm{~Hz}, \mathrm{~J}=1.2 \mathrm{~Hz}$, $\left.1 \mathrm{H}, \mathrm{py}^{4}\right), 5.40\left(\mathrm{~d}, \mathrm{~J}=8.3 \mathrm{~Hz}, 2 \mathrm{H}, \mathrm{py}^{3,5}\right), 2.17-2.02\left(\mathrm{~m}, 8 \mathrm{H}, \mathrm{CH}_{2}\right), 1.37-1.19\left(\mathrm{~m}, 12 \mathrm{H}, \mathrm{CH}_{3}\right) .{ }^{13} \mathrm{C}\left\{{ }^{1} \mathrm{H}\right\}$ $\operatorname{NMR}\left(\delta, \mathrm{CD}_{2} \mathrm{Cl}_{2}, 20^{\circ} \mathrm{C}\right.$ ): 221.7 (bt, CO), 208.4 (t, J = 7.0 Hz, CO), 164.5 (vt, J = $10.5 \mathrm{~Hz}, \mathrm{py}^{2,6}$ ), 140.7 (Ph), $136.6\left(\mathrm{py}^{4}\right), 130.7(\mathrm{Ph}), 129.3(\mathrm{Ph}), 128.4(\mathrm{Ph}), 100.8\left(\mathrm{py}^{3,5}\right), 29.0\left(\mathrm{vt}, \mathrm{J}=12.7 \mathrm{~Hz}, \mathrm{CH}_{2}\right.$ ), $9.4(\mathrm{~s}$, $\left.\mathrm{CH}_{3}\right) \cdot{ }^{31} \mathrm{P}\left\{{ }^{1} \mathrm{H}\right\} \operatorname{NMR}\left(\delta, \mathrm{CD}_{2} \mathrm{Cl}_{2}, 20^{\circ} \mathrm{C}\right): 112.7\left({ }^{1} \mathrm{~J}_{\mathrm{W}-\mathrm{p}}=328.6 \mathrm{~Hz}\right)$. IR $\left(\mathrm{ATR}, \mathrm{cm}^{-1}\right): 1934\left(v_{\mathrm{CO}}\right), 1804\left(v_{\mathrm{CO}}\right)$.

[W(PNP-BIPOL)(CO) $\left.{ }_{3}\right]$ (4i). The product obtained were yellow solid in $93 \%$ yield. Anal. Calcd. for $\mathrm{C}_{32} \mathrm{H}_{21} \mathrm{WN}_{3} \mathrm{O}_{7} \mathrm{P}_{2}$ (805.32). C, 47.73; $\mathrm{H}, 2.63 ; \mathrm{N}, 5.22$. Found: $\mathrm{C}, 47.60 ; \mathrm{H}, 2.72 ; \mathrm{N}, 5.13 .{ }^{1} \mathrm{H}$ NMR $(\delta$, acetone- $\left.\mathrm{d}_{6}, 20^{\circ} \mathrm{C}\right): 9.26(\mathrm{~s}, 2 \mathrm{H}, \mathrm{NH}), 7.68(\mathrm{dd}, \mathrm{J}=7.6 \mathrm{~Hz}, \mathrm{~J}=1.6 \mathrm{~Hz}, 4 \mathrm{H}, \mathrm{Ph}), 7.55-7.47(\mathrm{~m}, 5 \mathrm{H}, \mathrm{Ph}$, $\left.\mathrm{py}^{4}\right), 7.45-7.38(\mathrm{~m}, 8 \mathrm{H}, \mathrm{Ph}), 6.67\left(\mathrm{~d}, \mathrm{~J}=8 \mathrm{~Hz}, 2 \mathrm{H}, \mathrm{py}{ }^{3,5}\right) .{ }^{13} \mathrm{C}\left\{{ }^{1} \mathrm{H}\right\}$ NMR $\left(\delta\right.$, acetone- $\left.\mathrm{d}_{6}, 20{ }^{\circ} \mathrm{C}\right): 215.5(\mathrm{t}$, $\mathrm{J}=4.3 \mathrm{~Hz}, \mathrm{CO}$ ), 201.4 (t, J = 10.1 Hz, CO), 160.7 (vt, J = 11.5 Hz, py ${ }^{2,6}$ ), 150.7 (vt, J = 4.4 Hz, Ph), $140.0\left(\mathrm{py}^{4}\right), 130.6(2 \mathrm{Ph}), 126.7(\mathrm{Ph}), 123.3(\mathrm{Ph}), 100.16\left(\mathrm{vt}, \mathrm{J}=3.9 \mathrm{~Hz}, \mathrm{py},{ }^{3,5}\right) .{ }^{31} \mathrm{P}\left\{{ }^{1} \mathrm{H}\right\} \mathrm{NMR}(\delta$, acetone- $\left.\mathrm{d}_{6}, 20^{\circ} \mathrm{C}\right): 188.2\left({ }^{1} \mathrm{~J}_{\mathrm{w}-\mathrm{p}}=494.1 \mathrm{~Hz}\right)$. IR $\left(\mathrm{ATR}, \mathrm{cm}^{-1}\right): 1979\left(v_{\mathrm{CO}}\right), 1858\left(v_{\mathrm{CO}}\right)$.

Crystal Structure Determination. X-ray diffraction data of $\mathbf{2 a}, \mathbf{2 b} \cdot \mathrm{CH}_{3} \mathrm{CN}$, and $\mathbf{2 d} \cdot \mathrm{CH}_{3} \mathrm{CN}$ were collected at $T=100 \mathrm{~K}$ in a dry stream of nitrogen on a Bruker Kappa APEX II diffractometer system using graphite-monochromatized Mo-Ka radiation $(\lambda=0.71073 \AA)$ and fine sliced $\varphi$ - and $\omega$ scans. Data were reduced to intensity values with SAINT and an absorption correction was applied with the multi-scan approach implemented in SADABS. ${ }^{7}$ The structures were solved by charge flipping using SUPERFLIP ${ }^{8}$ and refined against $F$ with JANA2006. ${ }^{9}$ Non-hydrogen atoms were refined anisotropically. The $\mathrm{H}$ atoms connected to $\mathrm{C}$ atoms were placed in calculated positions and thereafter refined as riding on the parent atoms. The $\mathrm{H}$ atoms of the amine groups were located in difference Fourier maps and freely refined. Molecular graphics were generated with the program MERCURY. ${ }^{10}$ Crystal data and experimental details are given in Table S1. 
Table S1. Details for the crystal structure determinations of $\mathbf{2 a}, \mathbf{2} \mathbf{b} \cdot \mathrm{CH}_{3} \mathrm{CN}$, and $\mathbf{2 d} \cdot \mathrm{CH}_{3} \mathrm{CN}$.

\begin{tabular}{|c|c|c|c|}
\hline & $2 a$ & 2b. $\mathrm{CH}_{3} \mathrm{CN}$ & 2d. $\mathrm{CH}_{3} \mathrm{CN}$ \\
\hline formula & $\mathrm{C}_{16} \mathrm{H}_{25} \mathrm{CrN}_{3} \mathrm{O}_{3} \mathrm{P}_{2}$ & $\mathrm{C}_{22} \mathrm{H}_{36} \mathrm{CrN}_{4} \mathrm{O}_{3} \mathrm{P}_{2}$ & $\mathrm{C}_{26} \mathrm{H}_{44} \mathrm{CrN}_{4} \mathrm{O}_{3} \mathrm{P}_{2}$ \\
\hline fw & 421.3 & 518.5 & 574.6 \\
\hline cryst.size, $\mathrm{mm}$ & $0.57 \times 0.49 \times 0.43$ & $0.75 \times 0.26 \times 0.12$ & $0.65 \times 0.41 \times 0.14$ \\
\hline color, shape & translucent red block & translucent yellow rod & translucent yellow block \\
\hline crystal system & monoclinic & monoclinic & monoclinic \\
\hline space group & $P 2_{1} / n$ (no. 14) & $P 2_{1} / n$ (no. 14) & $P 2_{1} / n$ (no. 14) \\
\hline$a, \AA$ & 9.7554(7) & $7.8872(3)$ & $8.7134(7)$ \\
\hline$b, \AA$ & $11.6087(8)$ & 16.2499(6) & $15.8895(13)$ \\
\hline$c, \AA$ & $17.5753(12)$ & $20.4084(8)$ & $21.7498(18)$ \\
\hline$\beta,{ }^{\circ}$ & 96.9255(17) & 99.9097(11) & 96.9245(19) \\
\hline$V, \AA^{3}$ & \begin{tabular}{|l|}
$1975.8(2)$ \\
\end{tabular} & $2576.64(17)$ & $2989.3(4)$ \\
\hline$T, \mathrm{~K}$ & 100 & 100 & 100 \\
\hline $\mathrm{Z,Z^{ \prime }}$ & 4,1 & 4,1 & 4,1 \\
\hline$\rho_{\text {calc }}, \mathrm{g} \mathrm{cm}^{-3}$ & 1.4159 & 1.3362 & 1.2768 \\
\hline$\mu, \mathrm{mm}^{-1}(\mathrm{MoK} \alpha)$ & 0.760 & 0.598 & 0.522 \\
\hline$F(000)$ & 880 & 1096 & 1224 \\
\hline absorption & multi-scan, 0.65-0.72 & Multi-scan, 0.83-0.93 & multi-scan, $0.77-0.93$ \\
\hline$\theta$ range, deg & $2.11-32.60$ & $1.61-30.26$ & $1.59-35.03$ \\
\hline no. of rflns measd & 50691 & 33609 & 60419 \\
\hline$R_{\text {int }}$ & 0.0238 & 0.0291 & 0.0526 \\
\hline no. of rflns unique & 7150 & 7515 & 13143 \\
\hline no. of rflns $1>3 \sigma(l)$ & 6589 & 6142 & 9373 \\
\hline no. of params $/$ & $234 / 0$ & $297 / 0$ & $333 / 0$ \\
\hline$R(I>3 \sigma(I))^{a}$ & 0.0221 & 0.0306 & 0.0380 \\
\hline$R$ (all data) & 0.0245 & 0.0421 & 0.0662 \\
\hline$w R(I>3 \sigma(I))$ & 0.0391 & 0.0373 & 0.0384 \\
\hline$w R$ (all data) & 0.0393 & 0.0384 & 0.0419 \\
\hline GooF & 2.59 & 1.86 & 1.31 \\
\hline Diff.Four.peaks & $-0.36 / 0.45$ & $-0.39 / 0.38$ & $-0.56 / 0.52$ \\
\hline
\end{tabular}




\section{Figures}

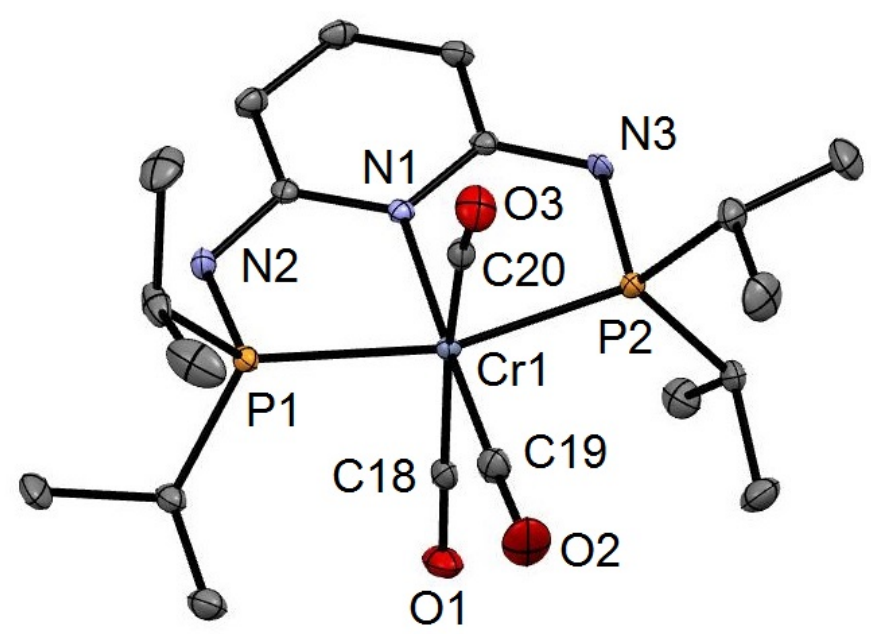

Figure S1. Structural view of $\left[\mathrm{Cr}(\mathrm{PNP}-\mathrm{Pr})(\mathrm{CO})_{3}\right] \cdot \mathrm{CH}_{3} \mathrm{CN}\left(\mathbf{2} \mathbf{b} \cdot \mathrm{CH}_{3} \mathrm{CN}\right)$ showing $50 \%$ thermal ellipsoids ( $\mathrm{H}$ atoms and $\mathrm{CH}_{3} \mathrm{CN}$ omitted for clarity). Selected bond lengths $(\AA)$ and bond angles $\left({ }^{\circ}\right)$ : Cr1-P1 2.2930(4), Cr1-P2 2.2782(4), Cr1-C18 1.857(1), Cr1-C19 1.813(1), Cr1-C20 1.874(1), Cr1-N1 2.139(1), P1-Cr1-P2 160.04(1), C18-Cr1-C20 164.53(6).

\section{References}

1 Kobayashi, Y., Hayashi, N., Kishi, Y., Org. Lett. 2002, 4, 411-414.

2 Slowinski, F., Aubert, C., Malacria, M., J. Org. Chem. 2003, 68, 378-386.

3 Schirmer, W.; Flörke, U.; Haupt, H. J. Z. Anorg. Allg. Chem. 1987, 545, 83-97.

4 Benito-Garagorri, D., Becker, E.; Wiedermann, J.; Lackner, W.; Pollak, M.; Mereiter, K.; Kisala, J.; Kirchner, K. Organometallics 2006, 25, 1900-1913.

5 Öztopcu, Ö.; Holzhacker, C.; Puchberger, M.; Weil, M.; Mereiter, K.; Veiros, L. F.; Kirchner, K. Organometallics 2013, 32, 3042-3052.

6 de Aguiar, S. R. M. M.; Stöger, B.; Pittenauer, E.; Allmaier, G.; Puchberger, M.; Veiros, L. F.; Kirchner, K. J. Organomet. Chem. 2014, 760, 74-83.

7 Bruker computer programs: APEX2, SAINT and SADABS (Bruker AXS Inc., Madison, WI, 2012).

8 Palatinus, L.; Chapuis, G.J. Appl. Cryst. 2007, 40, 786-790.

9 Petříček, V.; Dušek, M.; Palatinus, L. JANA2006, the crystallographic computing system. (Institute of Physics, Praha, Czeck Republic, 2006)

10 Macrae, C. F.; Edgington, P. R.; McCabe, P.; Pidcock, E.; Shields, G. P.; Taylor, R.; Towler M.; van de Streek, J. J. Appl. Cryst. 2006, 39, 453. 


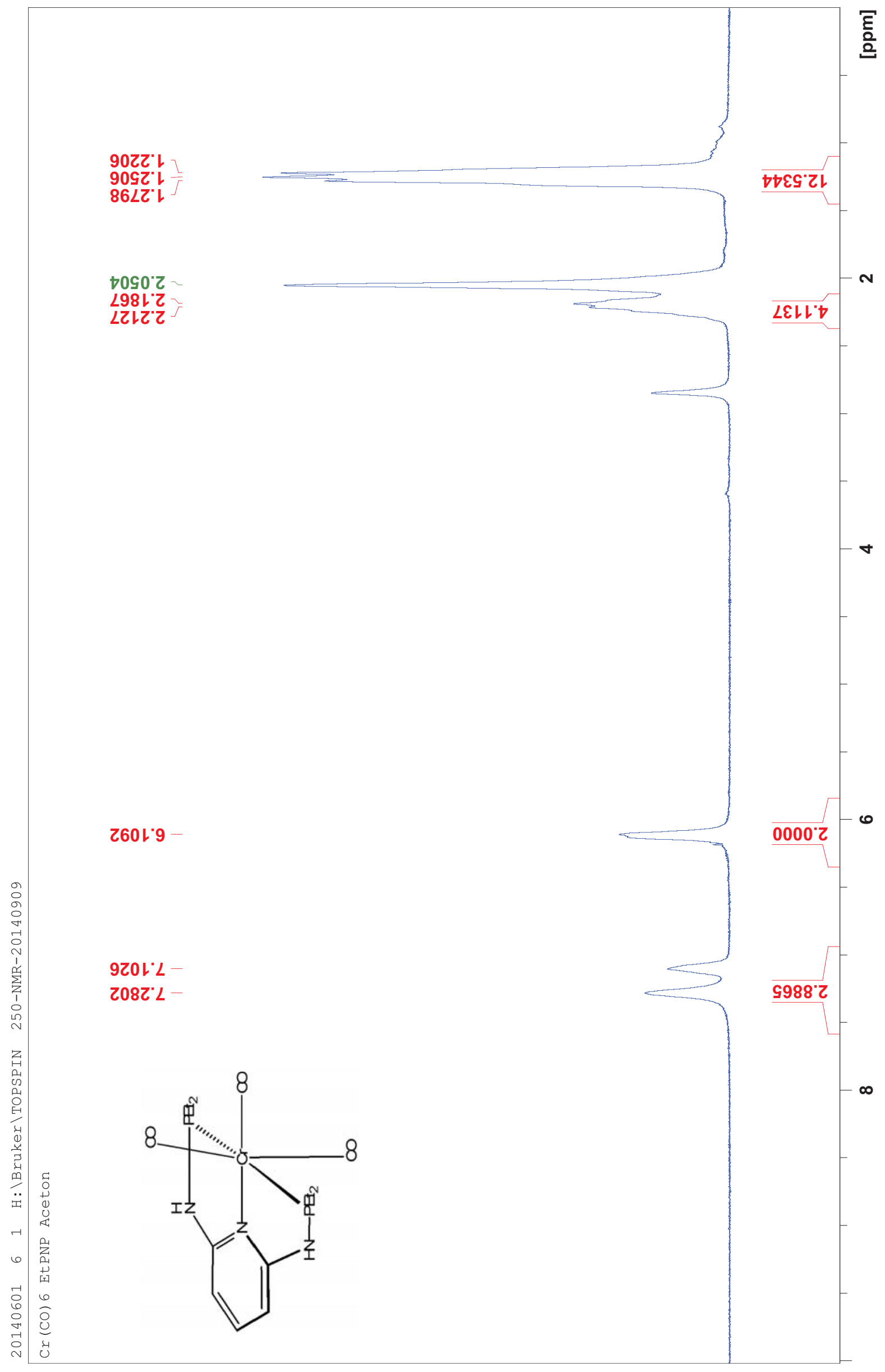




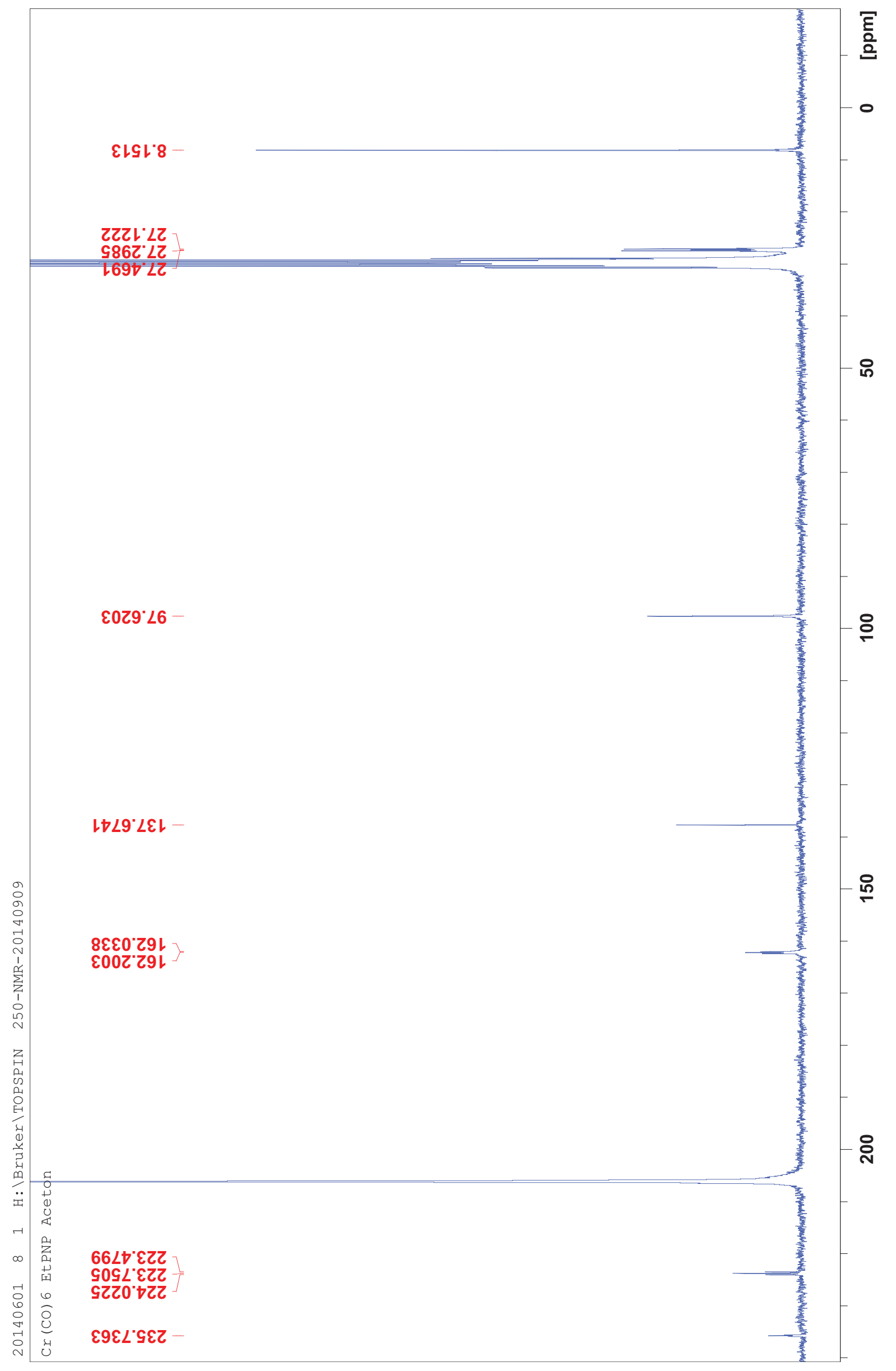




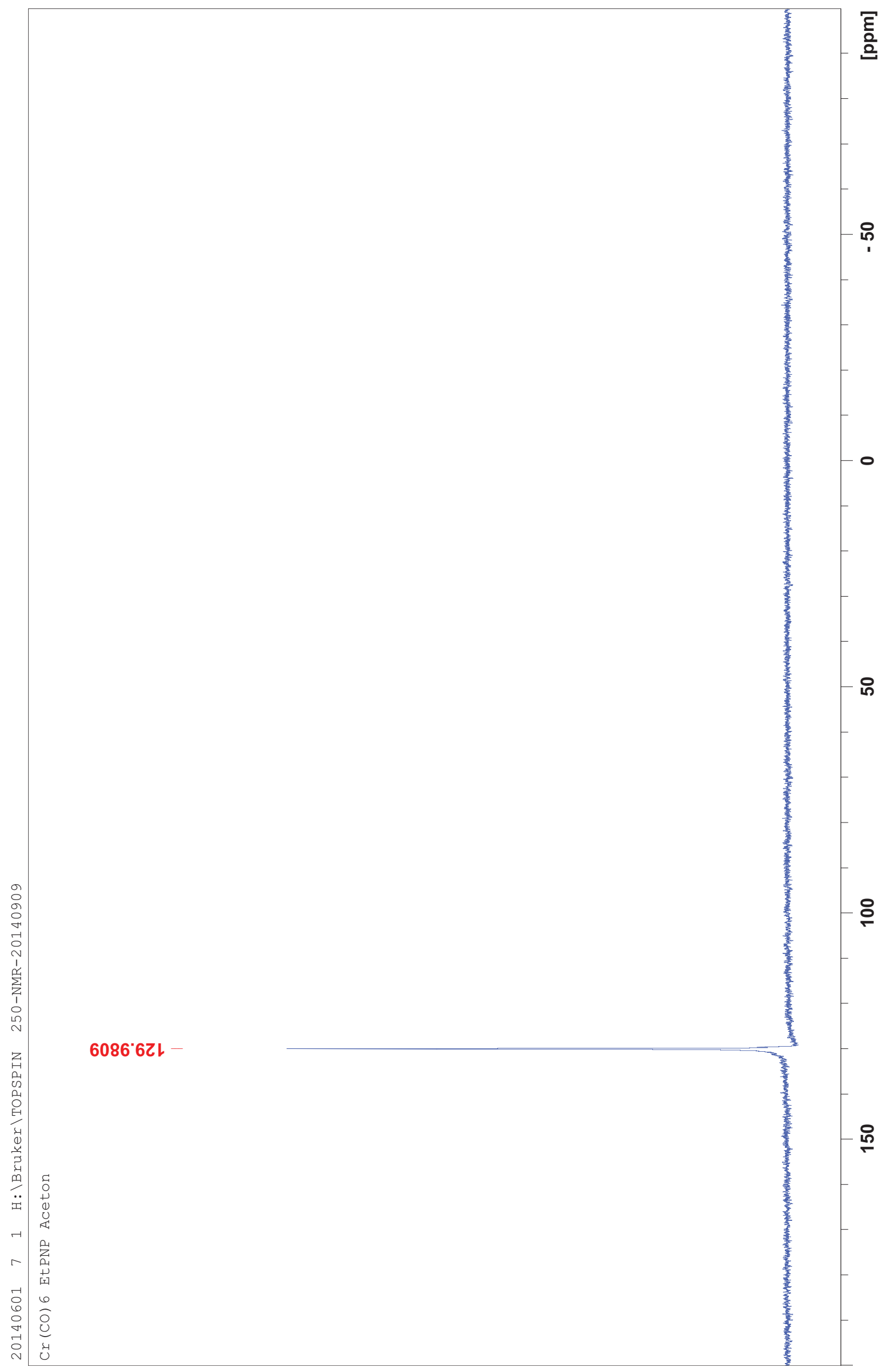




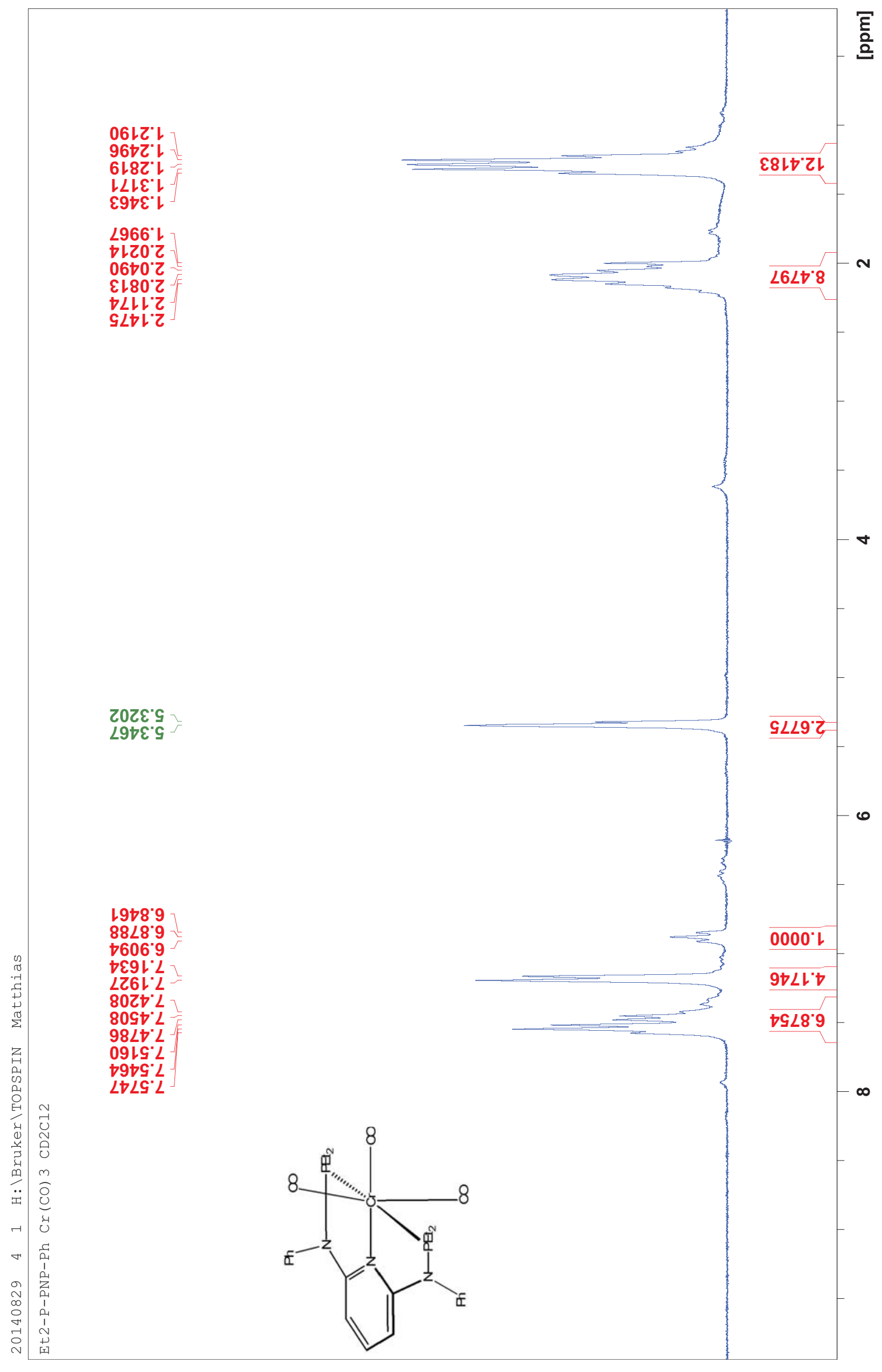




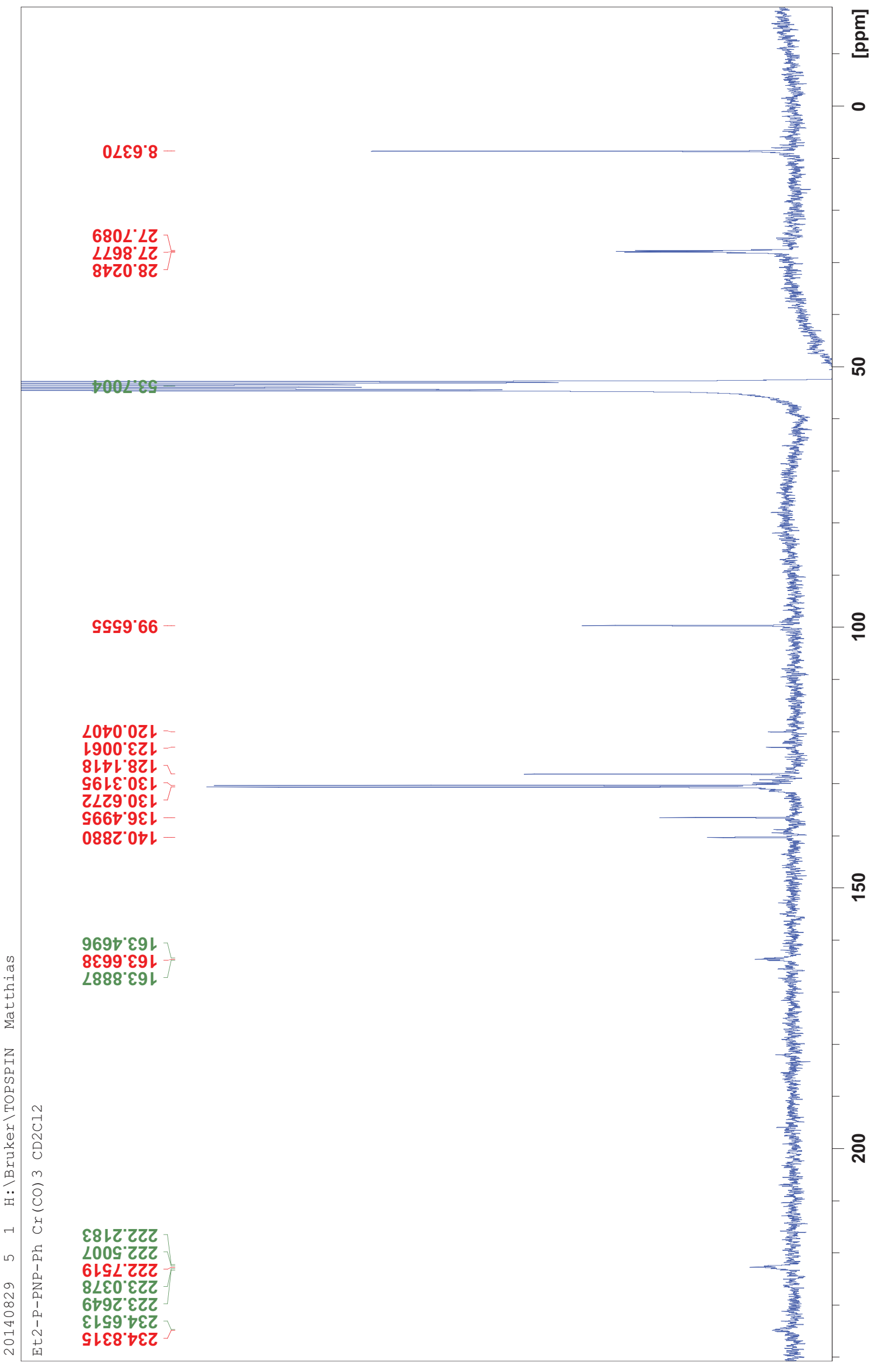




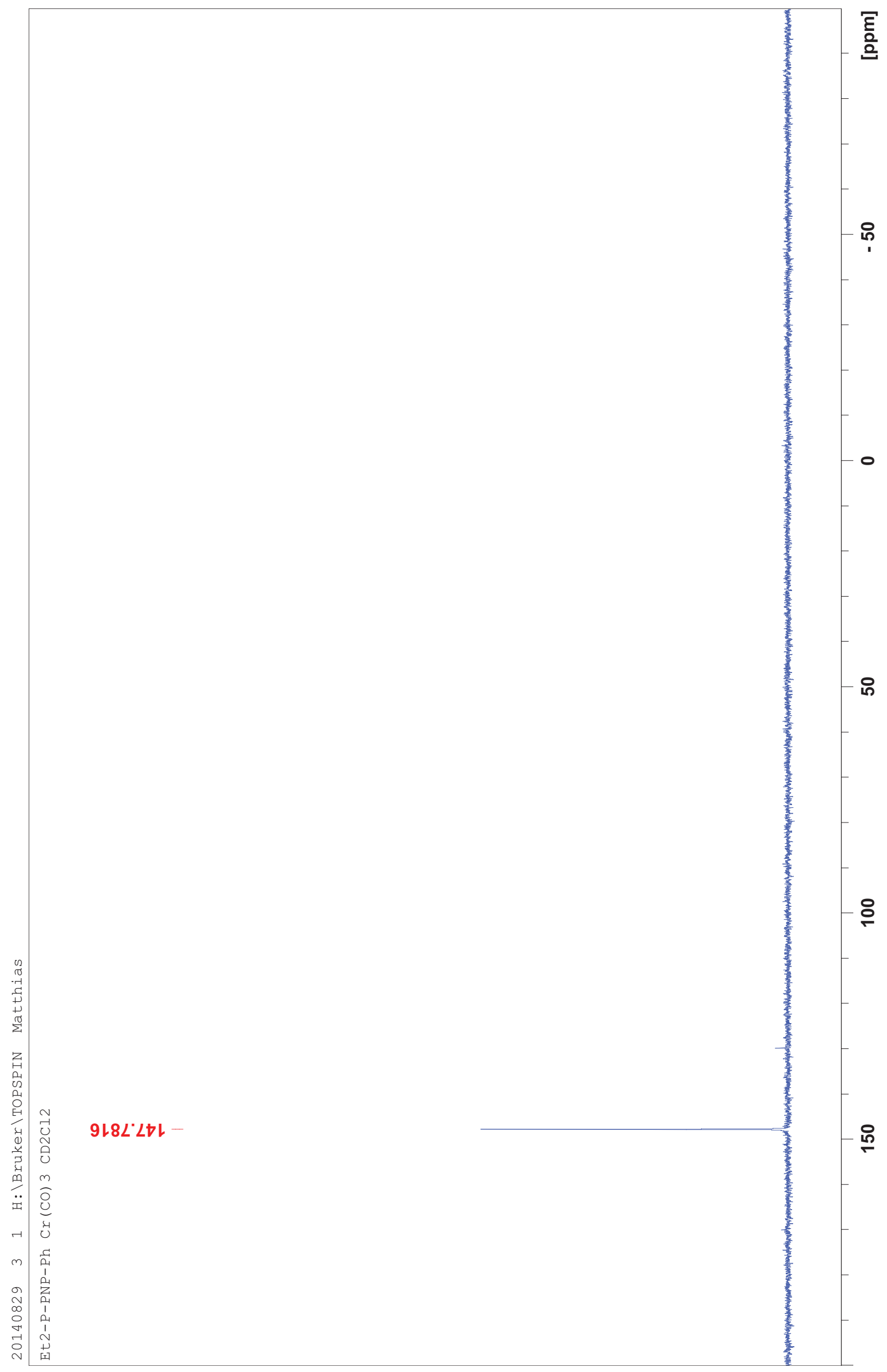




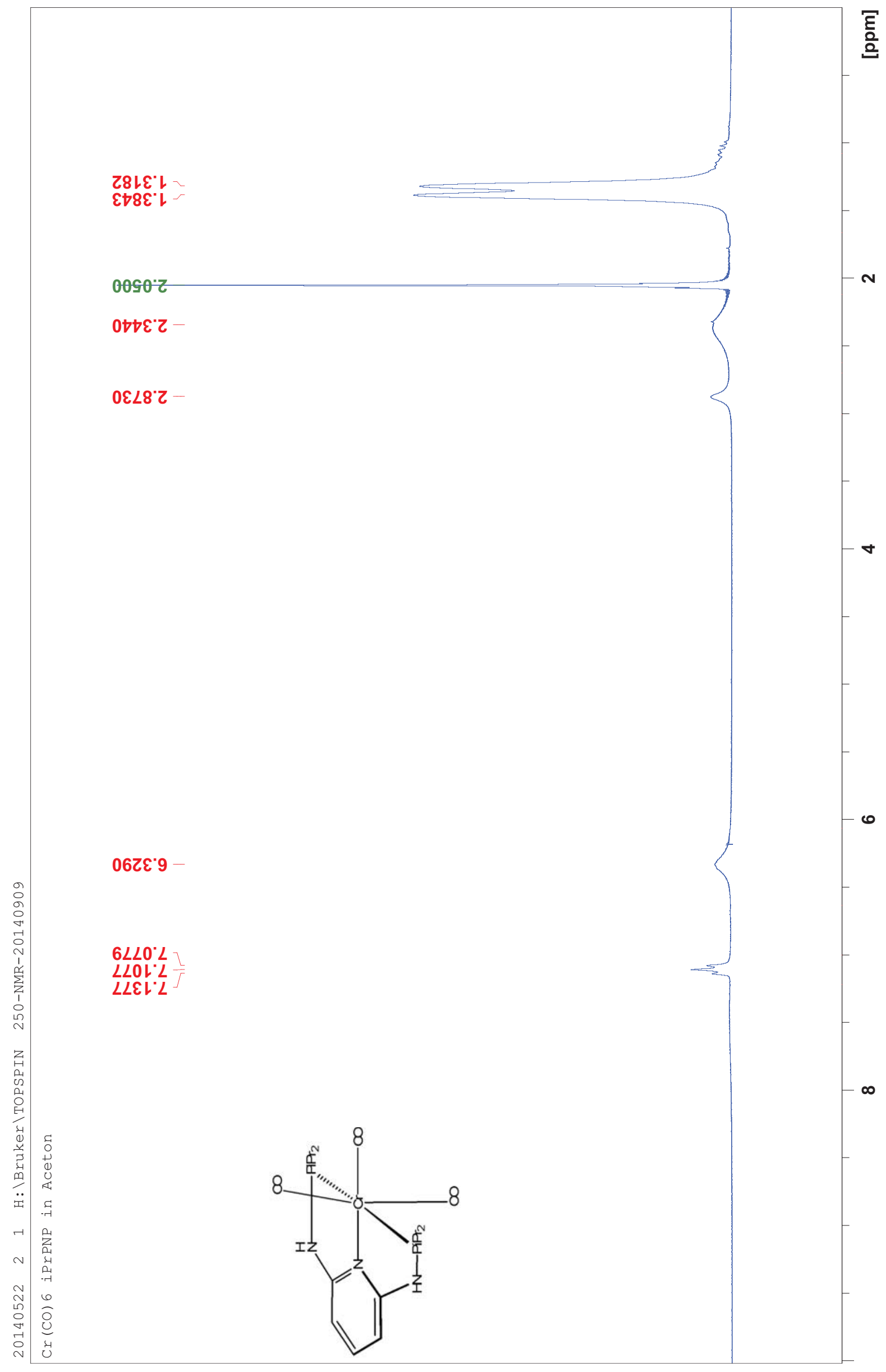




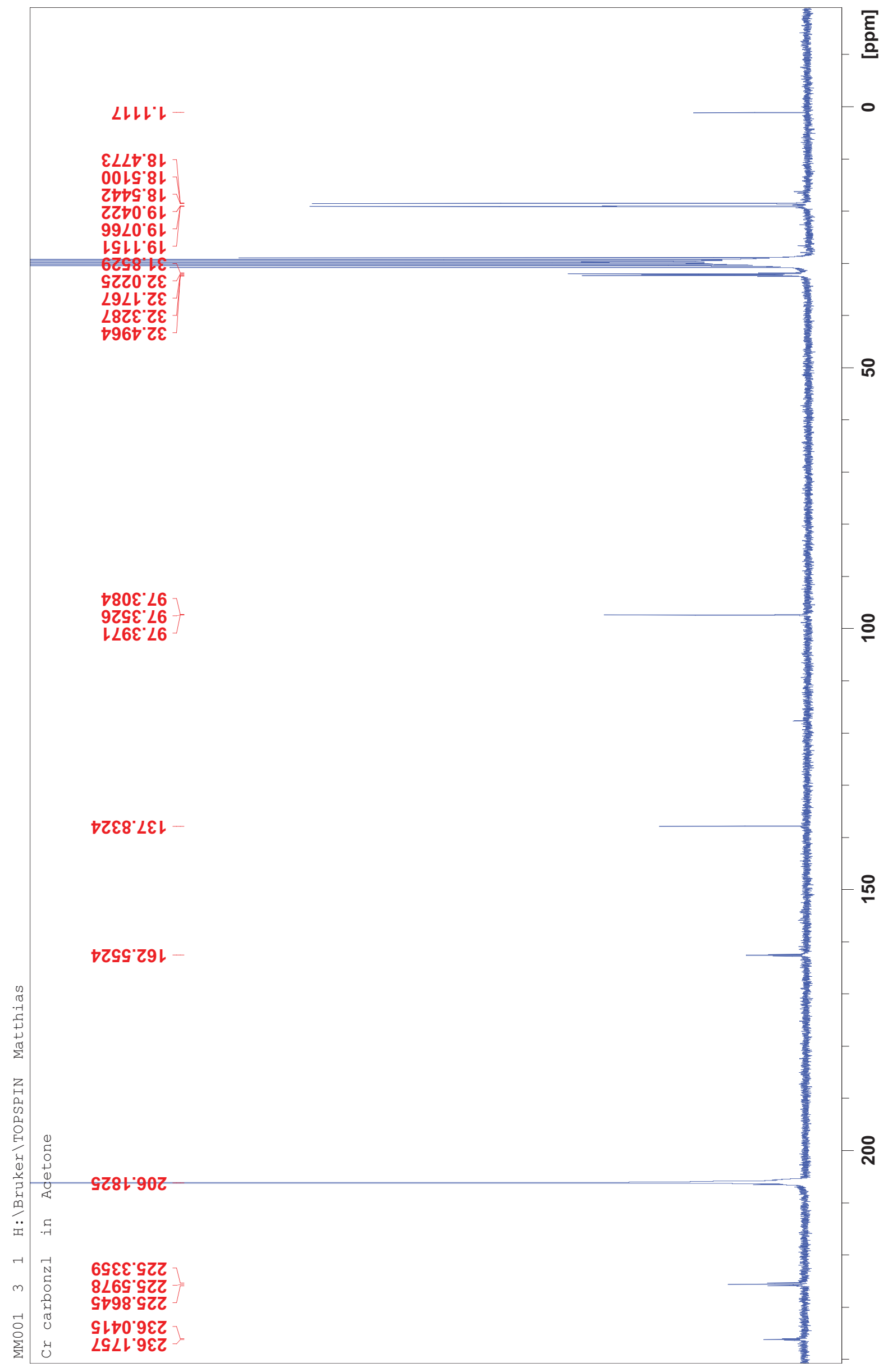




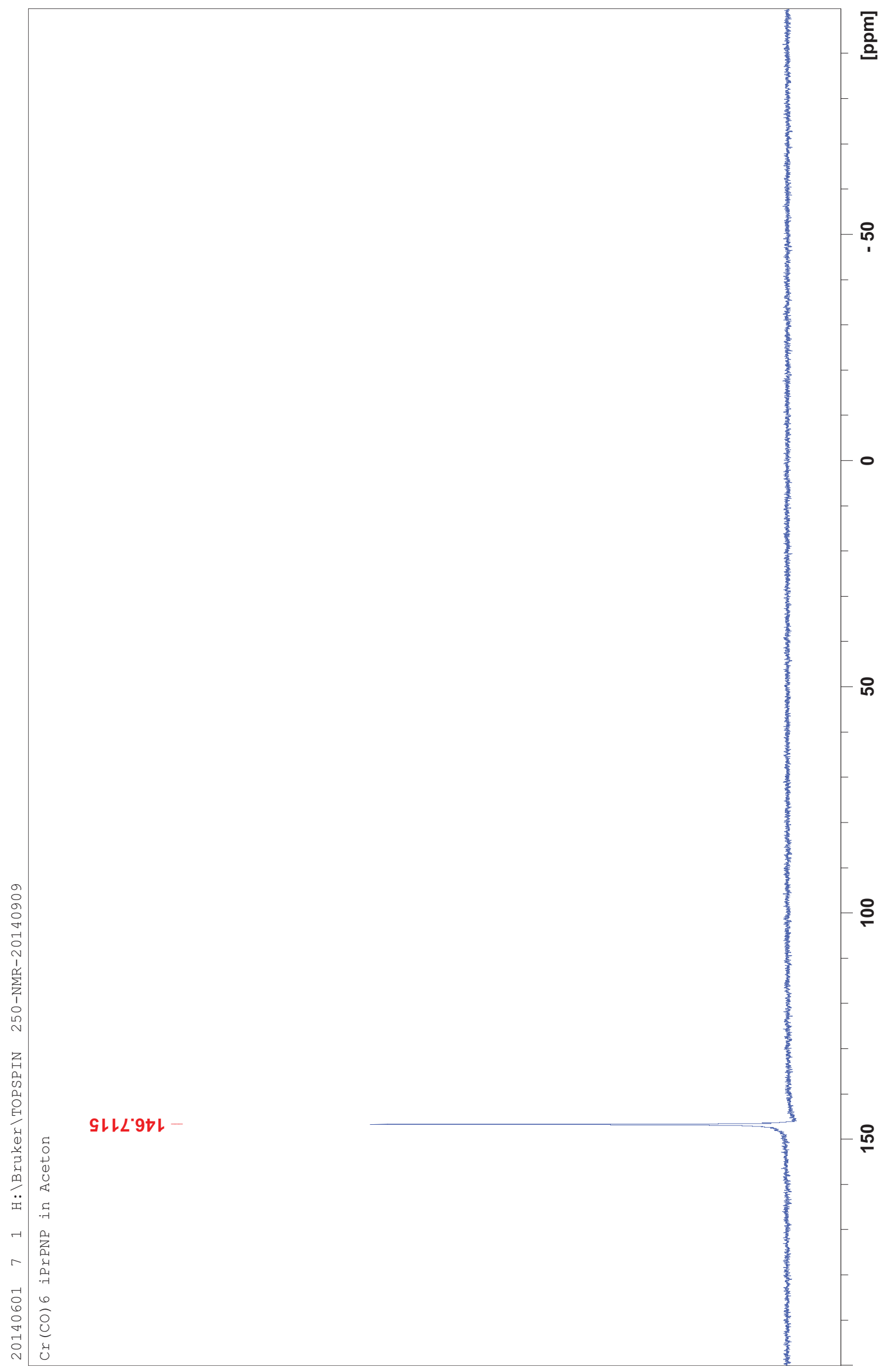




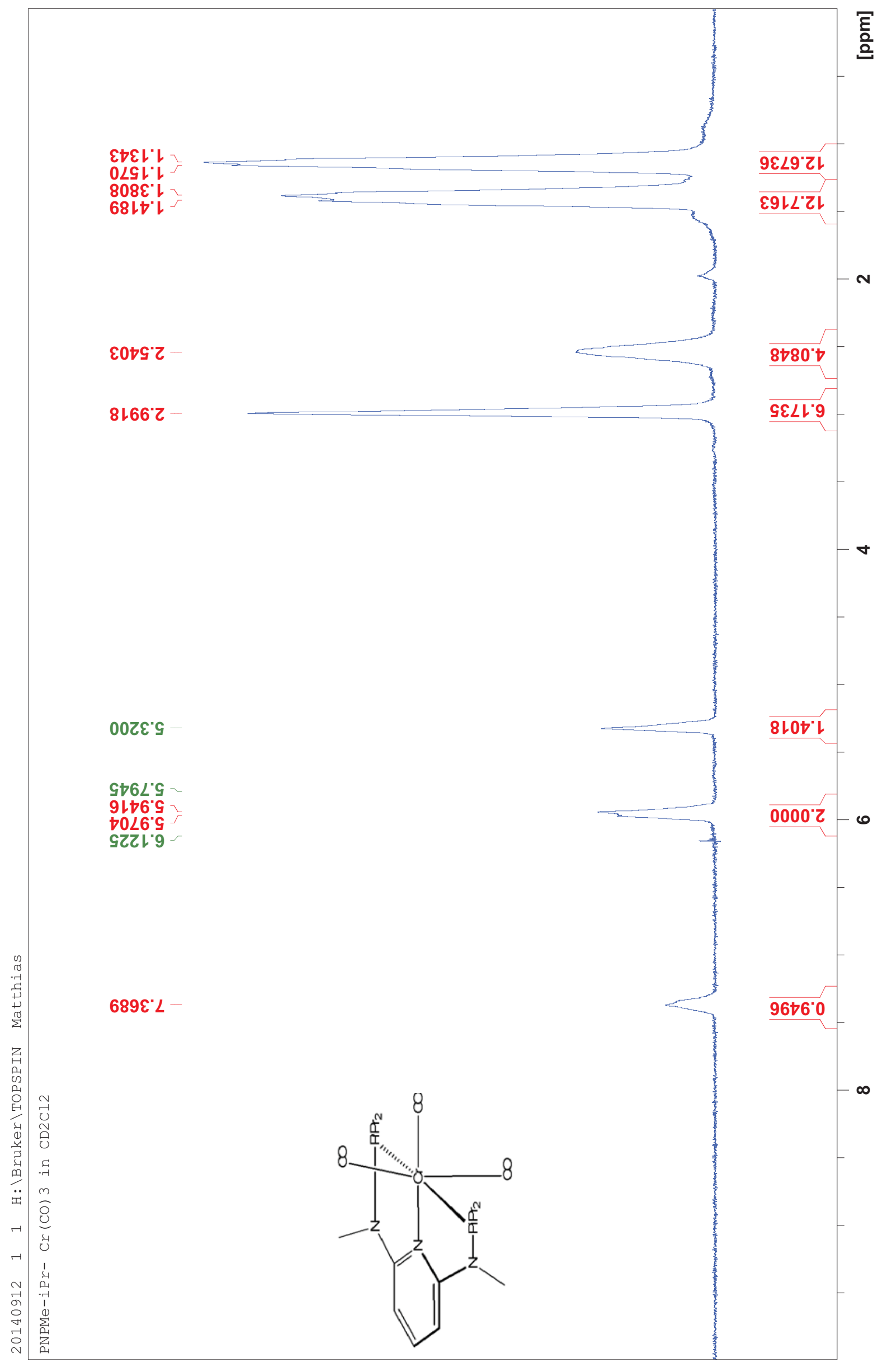




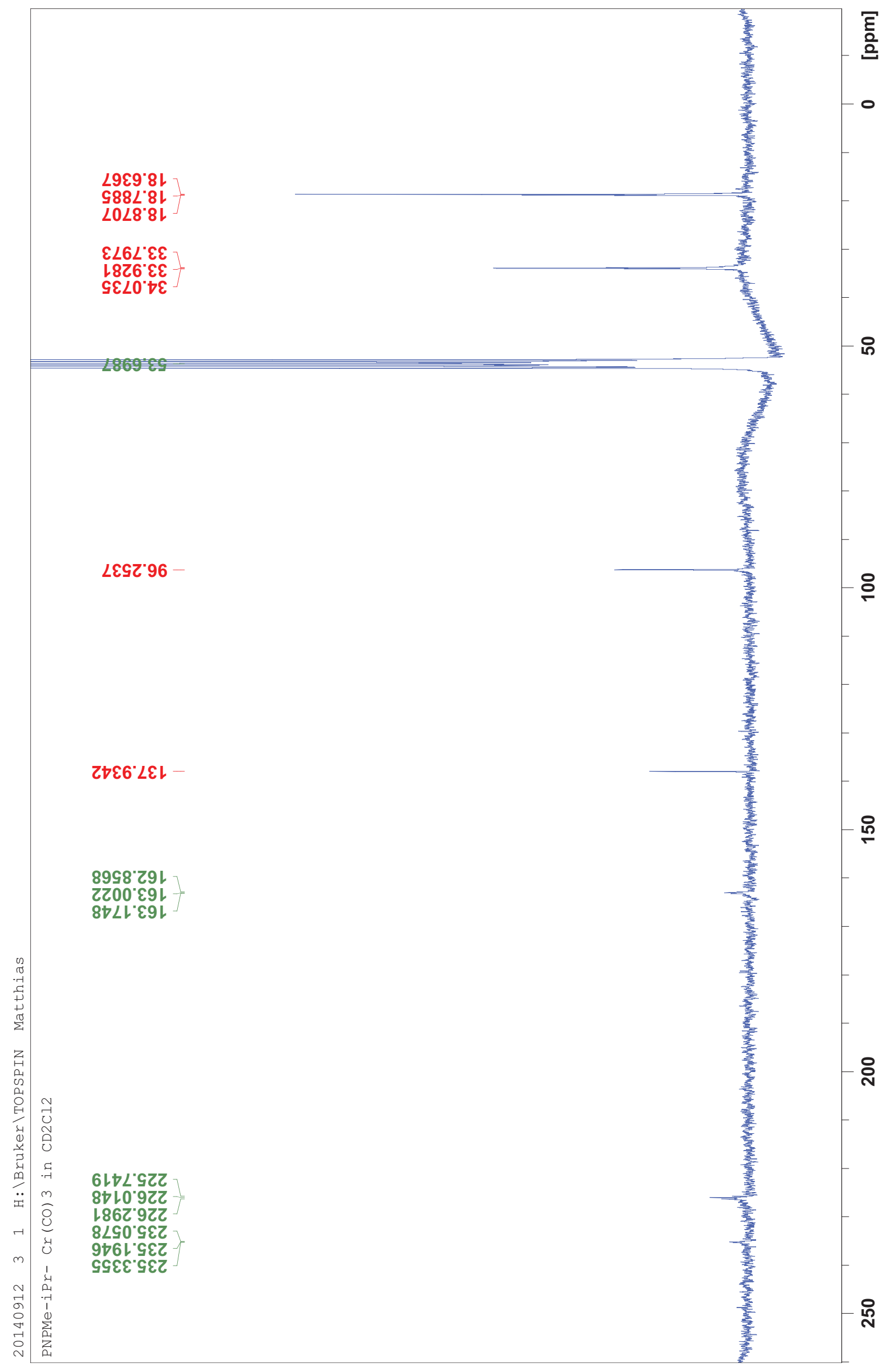




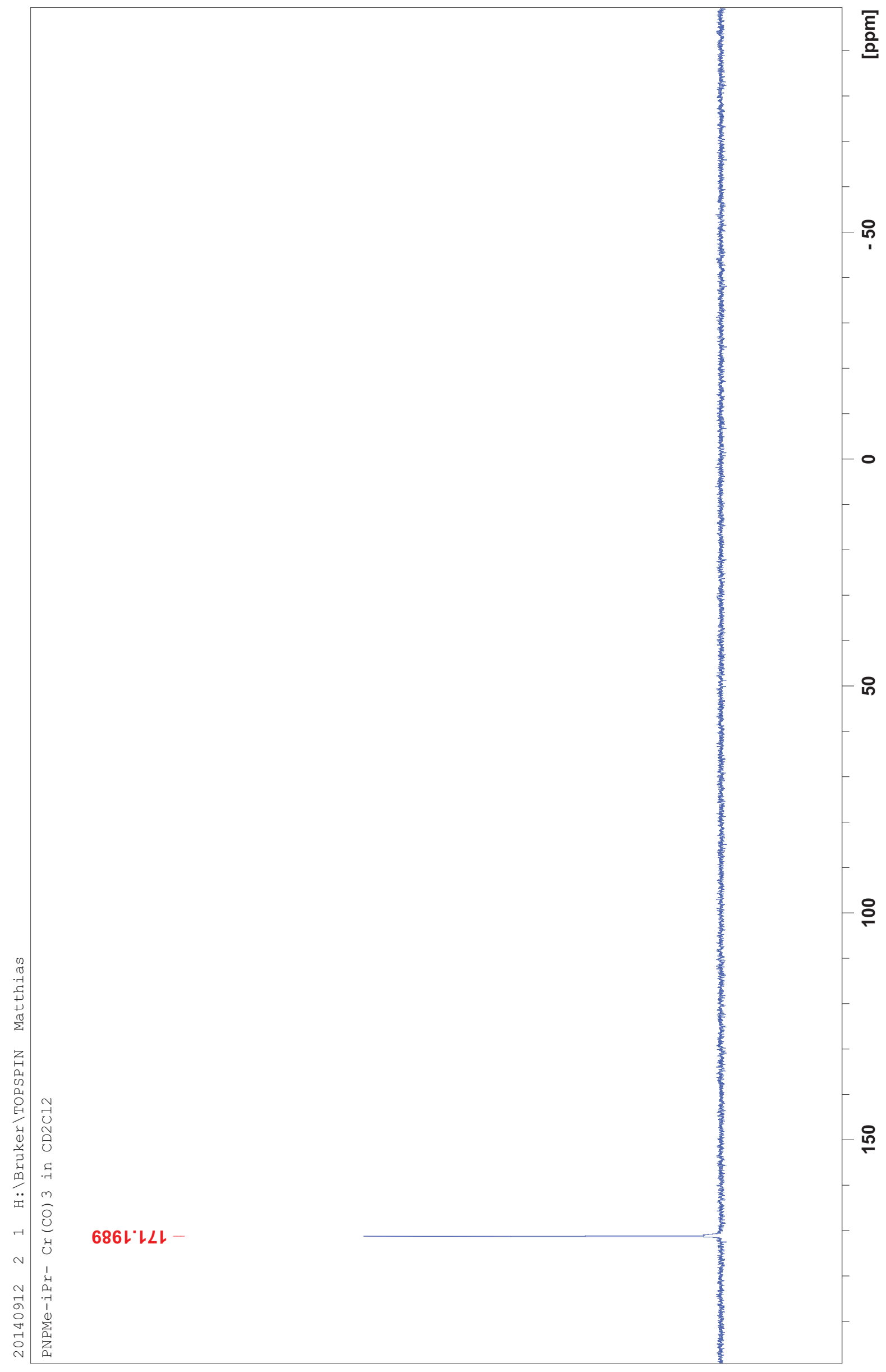




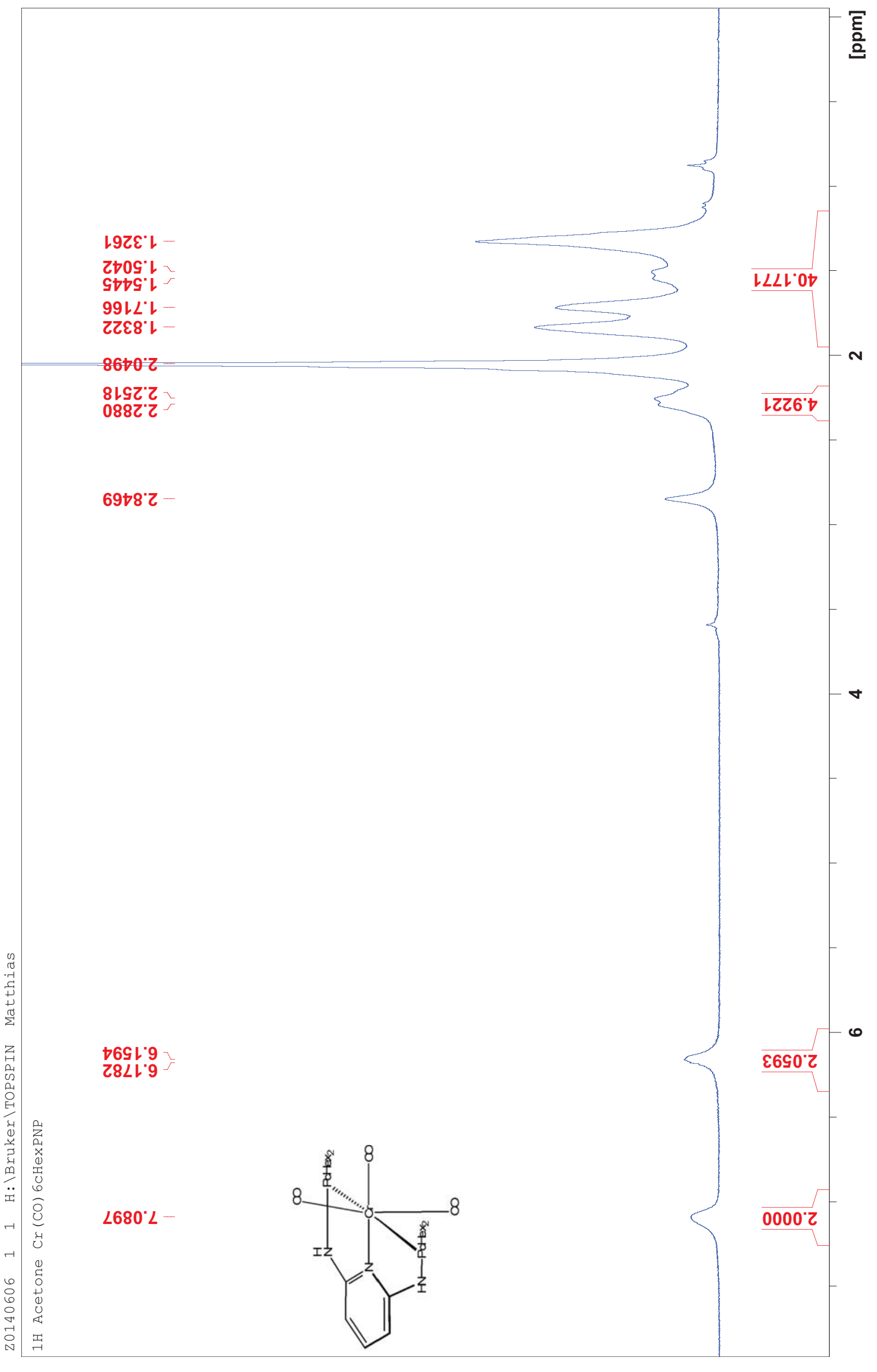




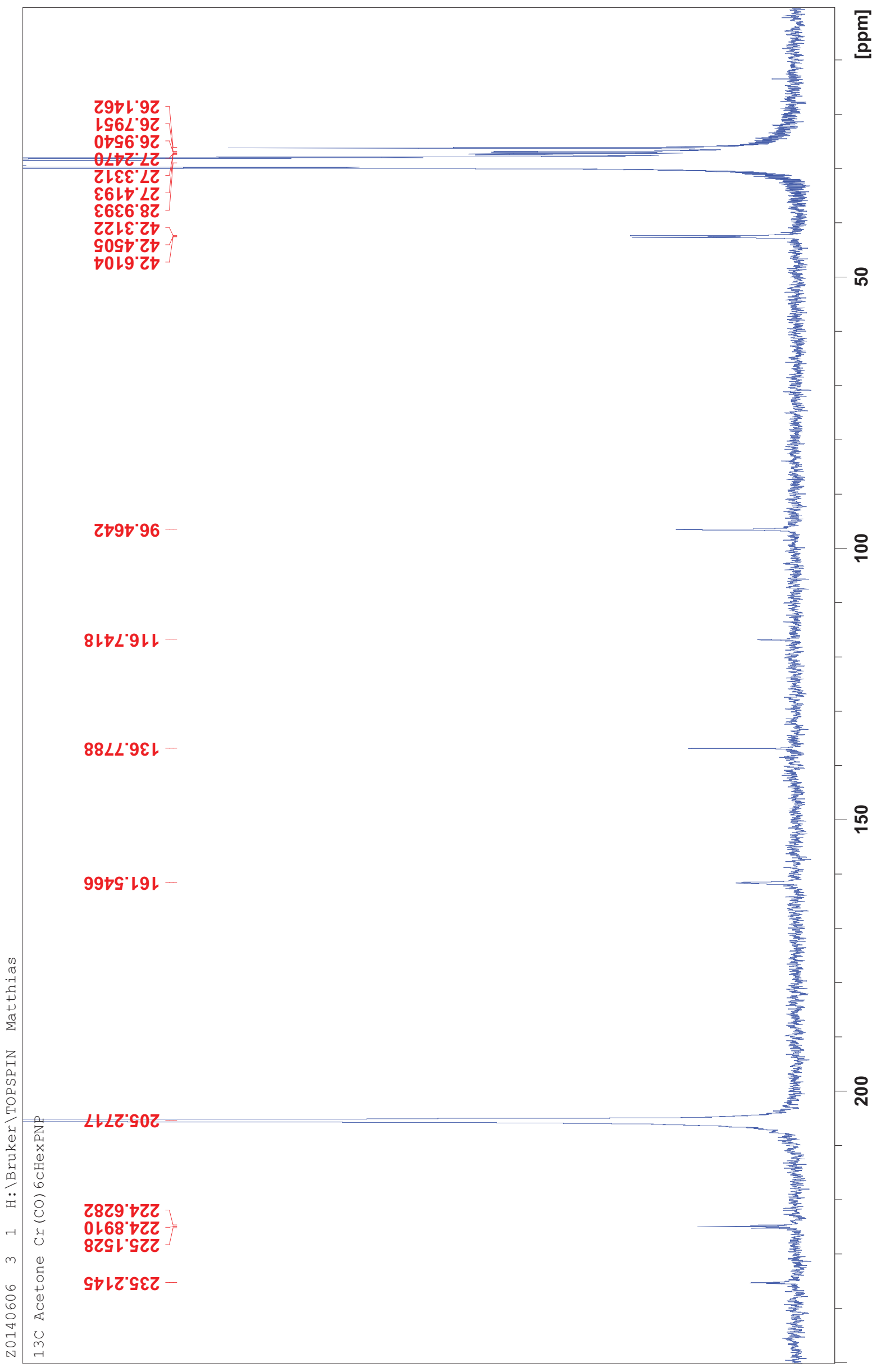




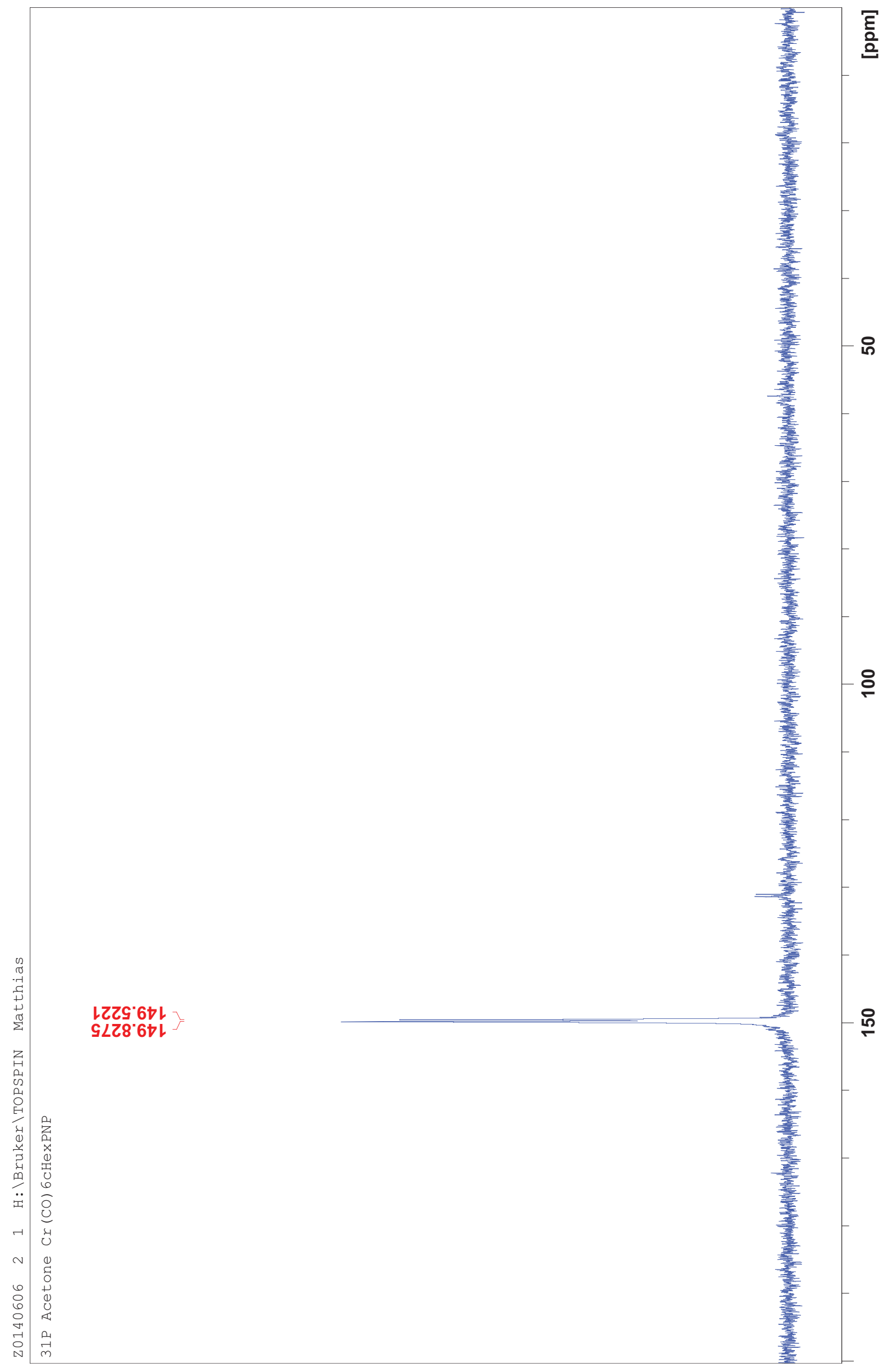




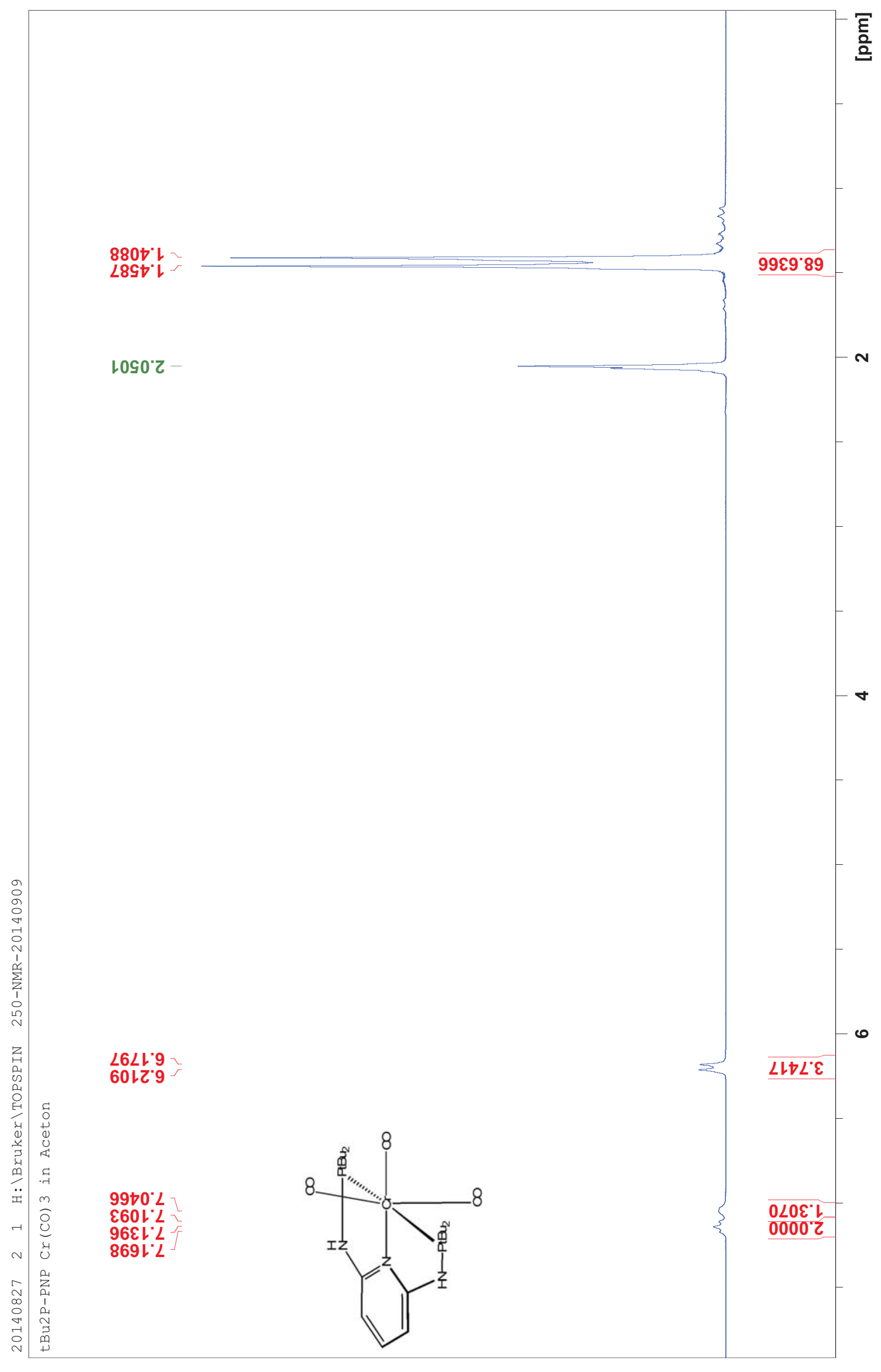




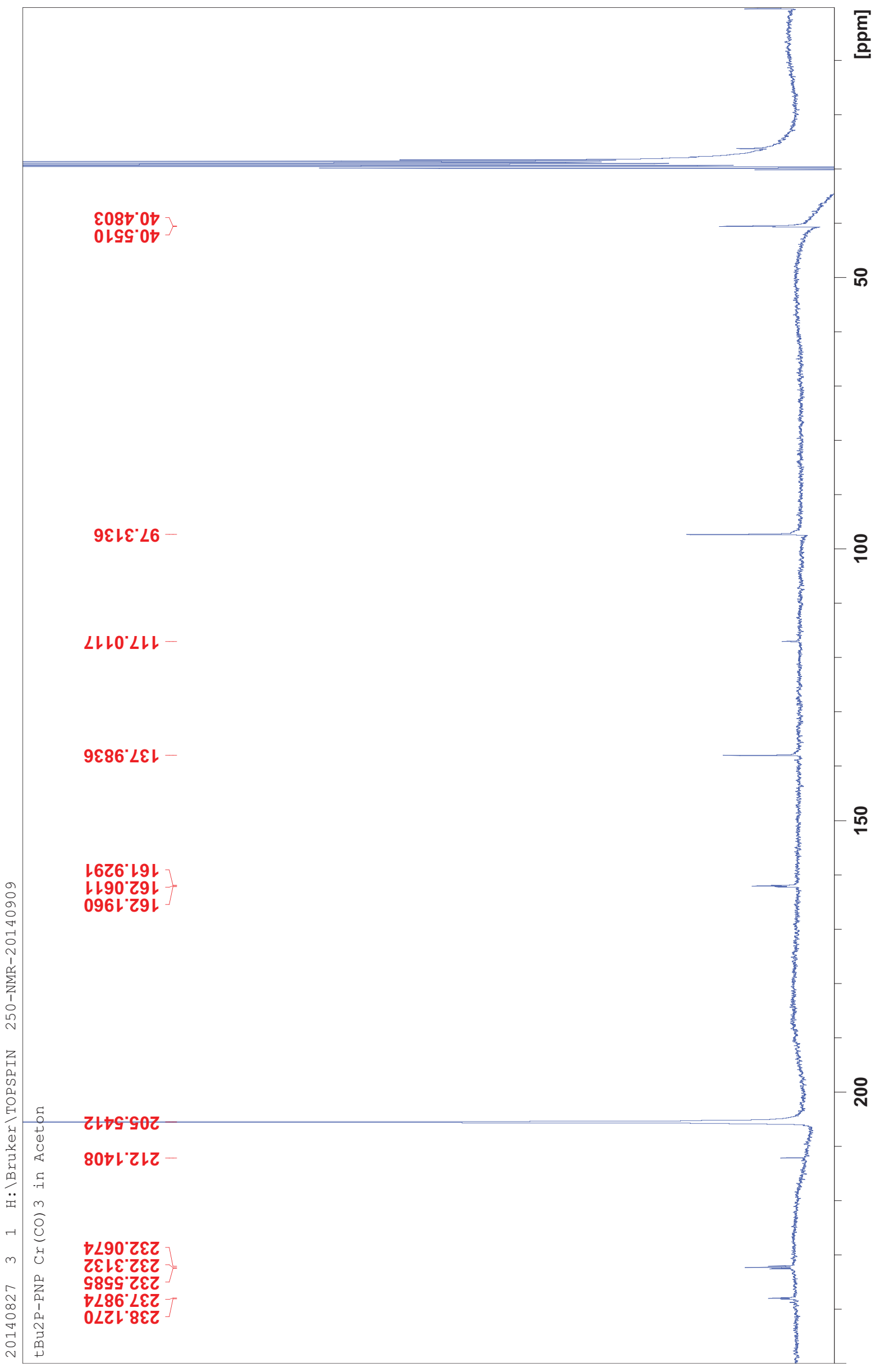




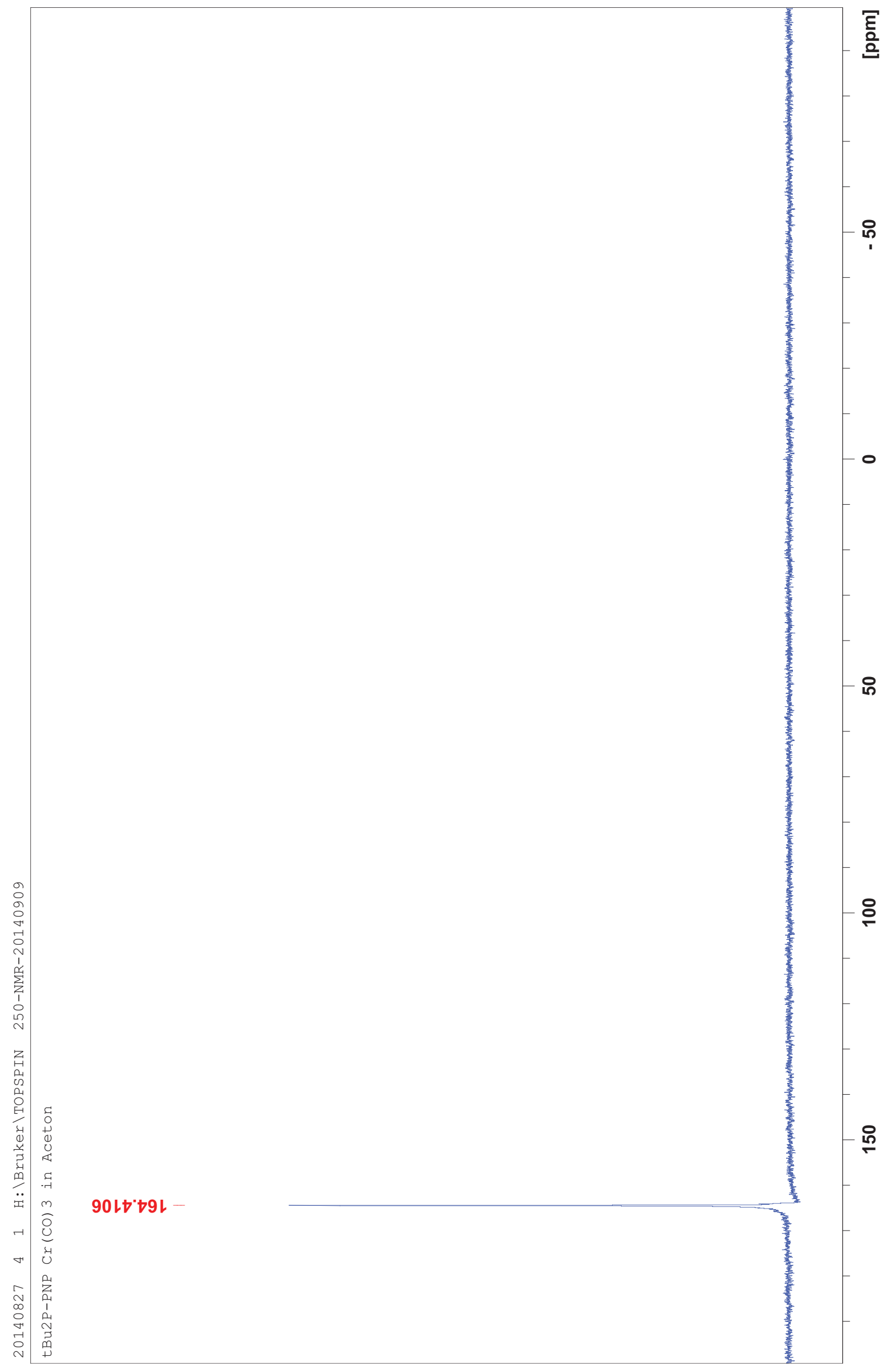




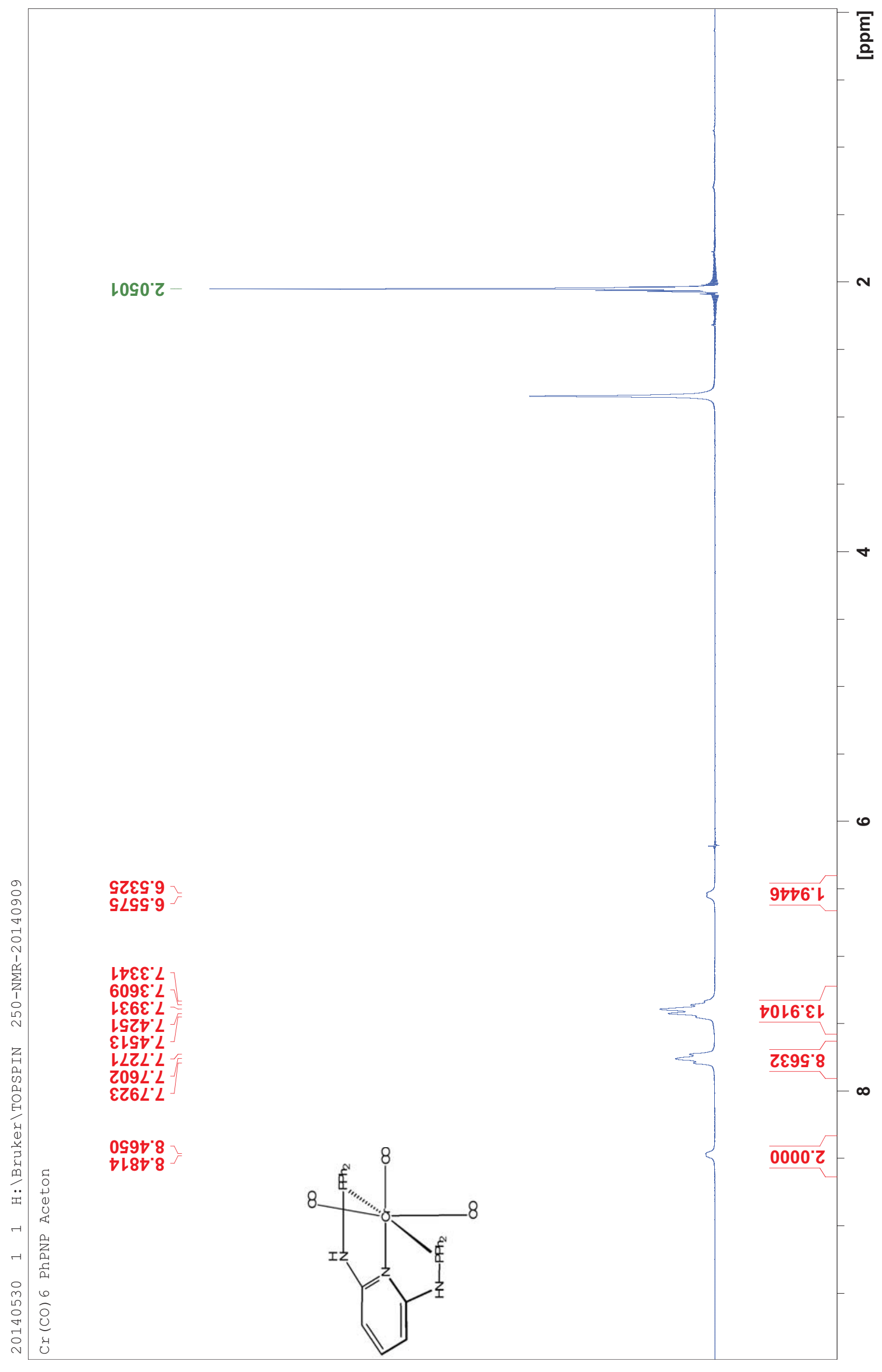




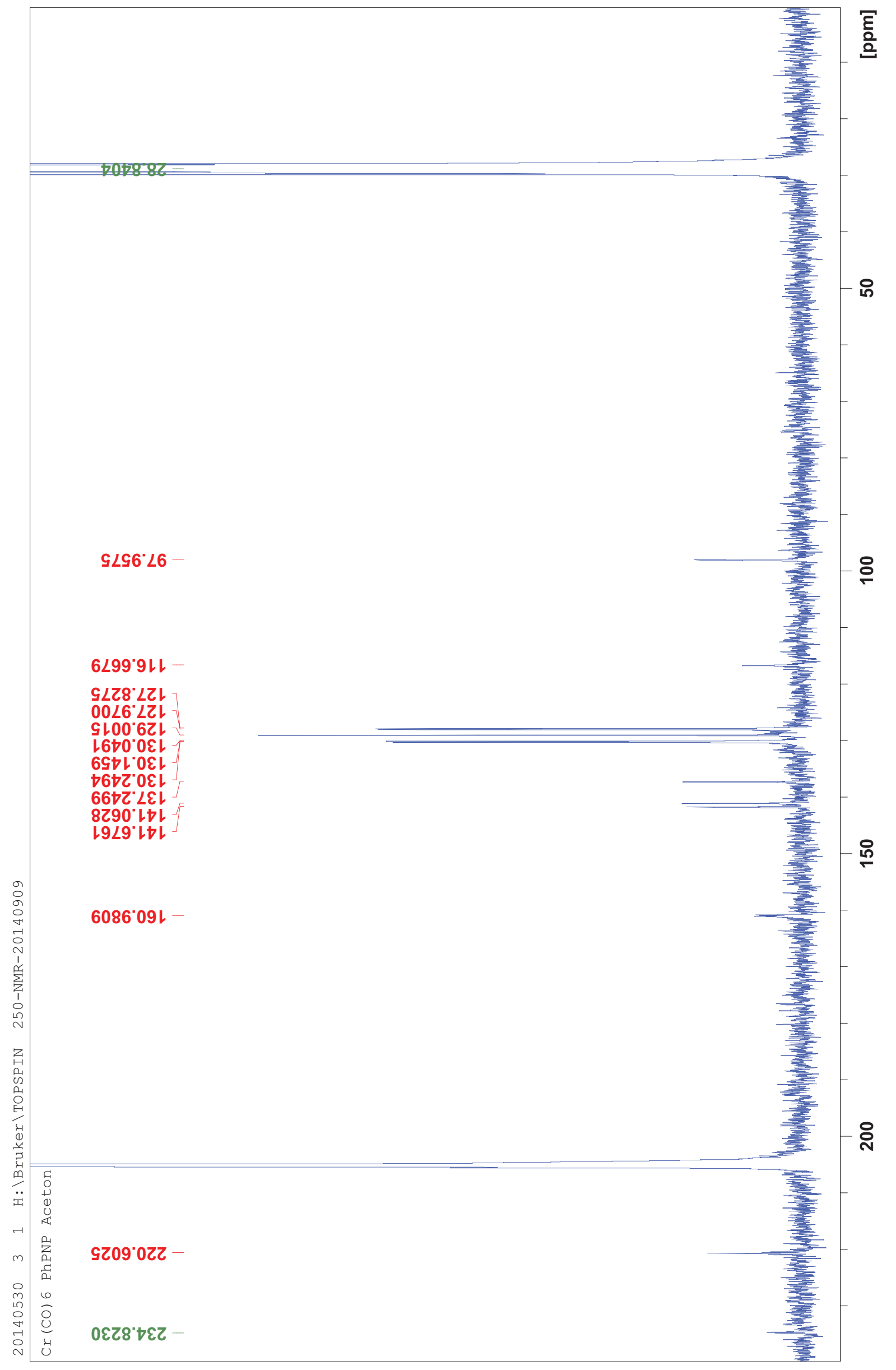




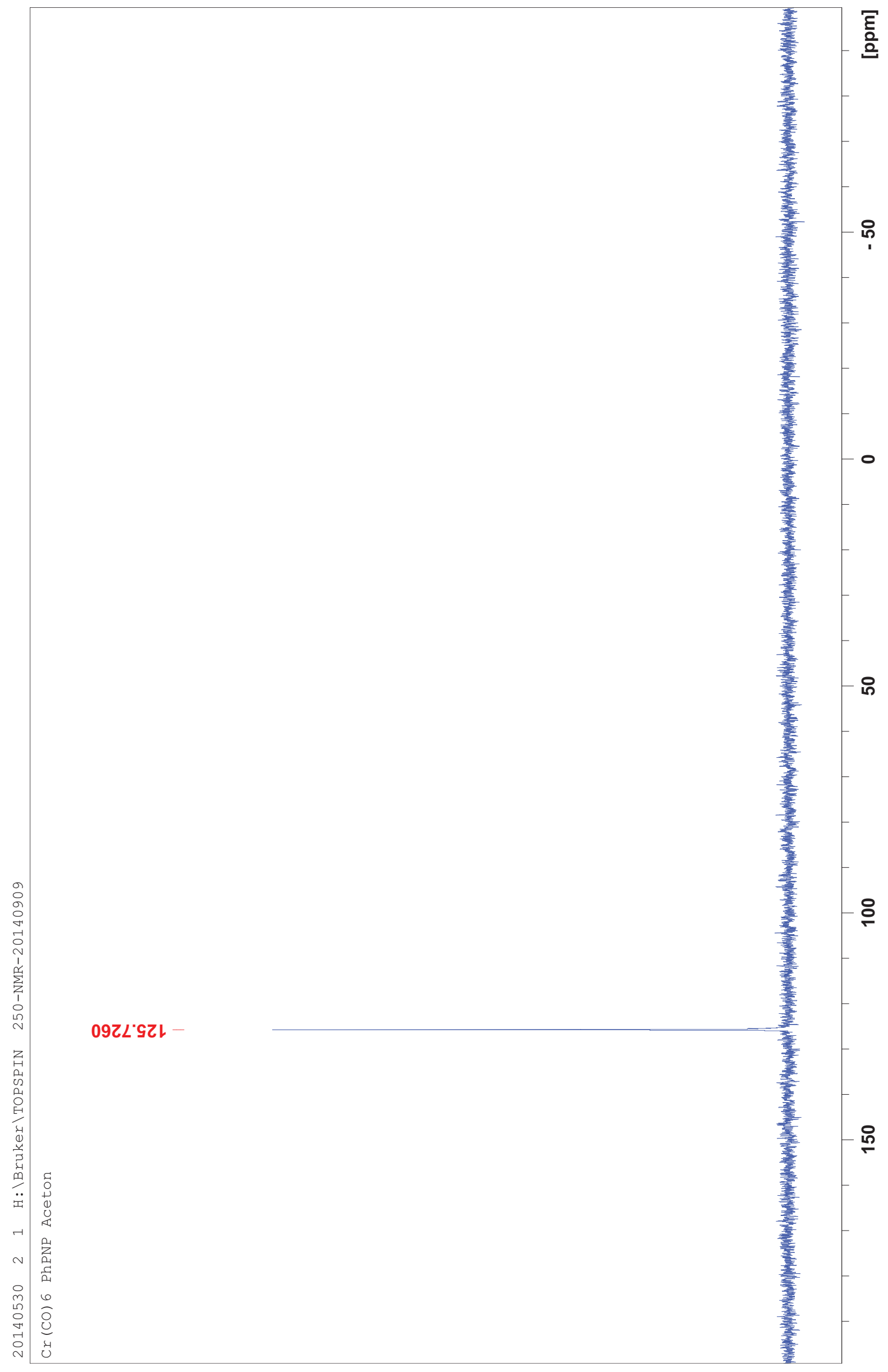




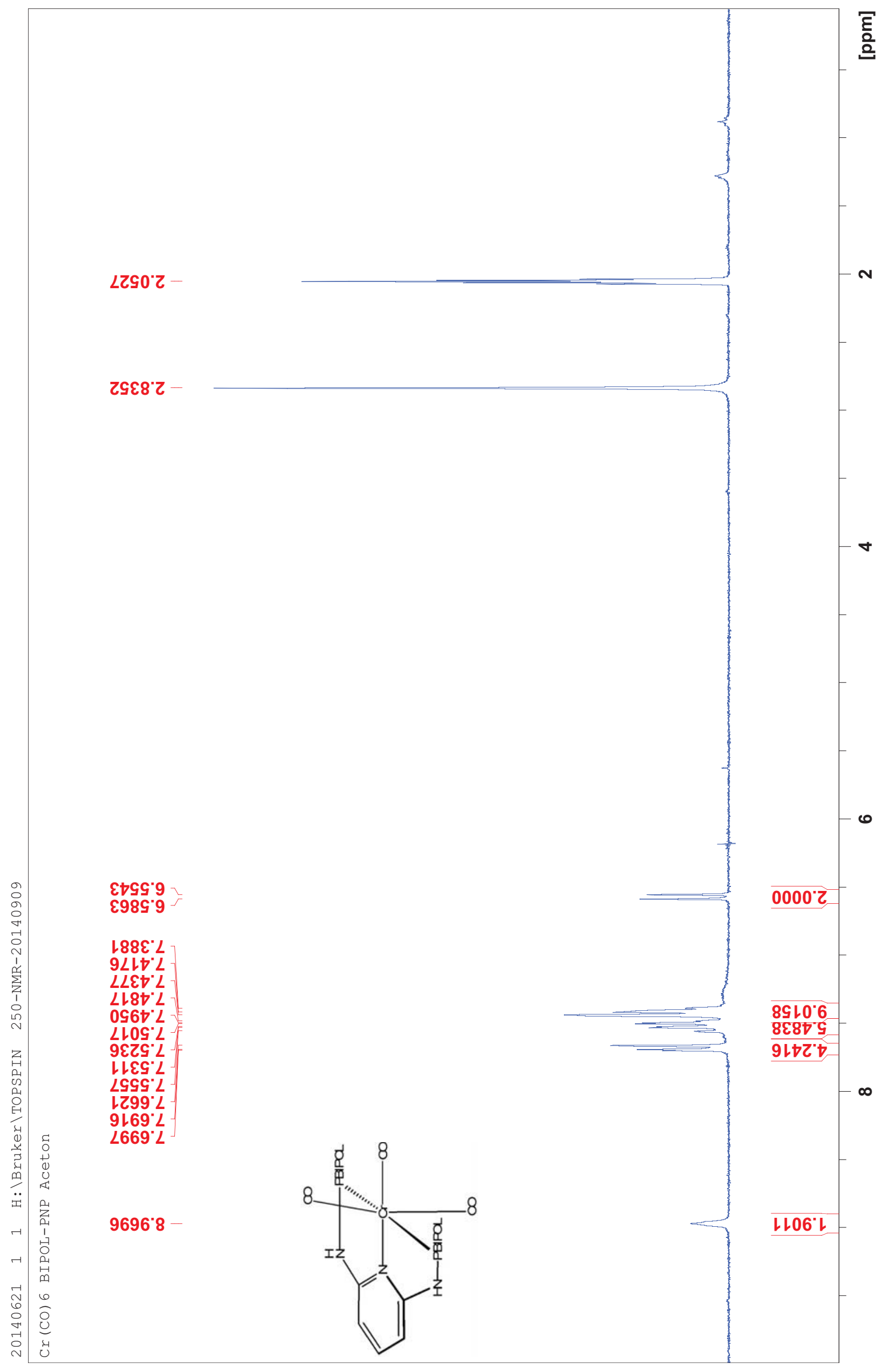




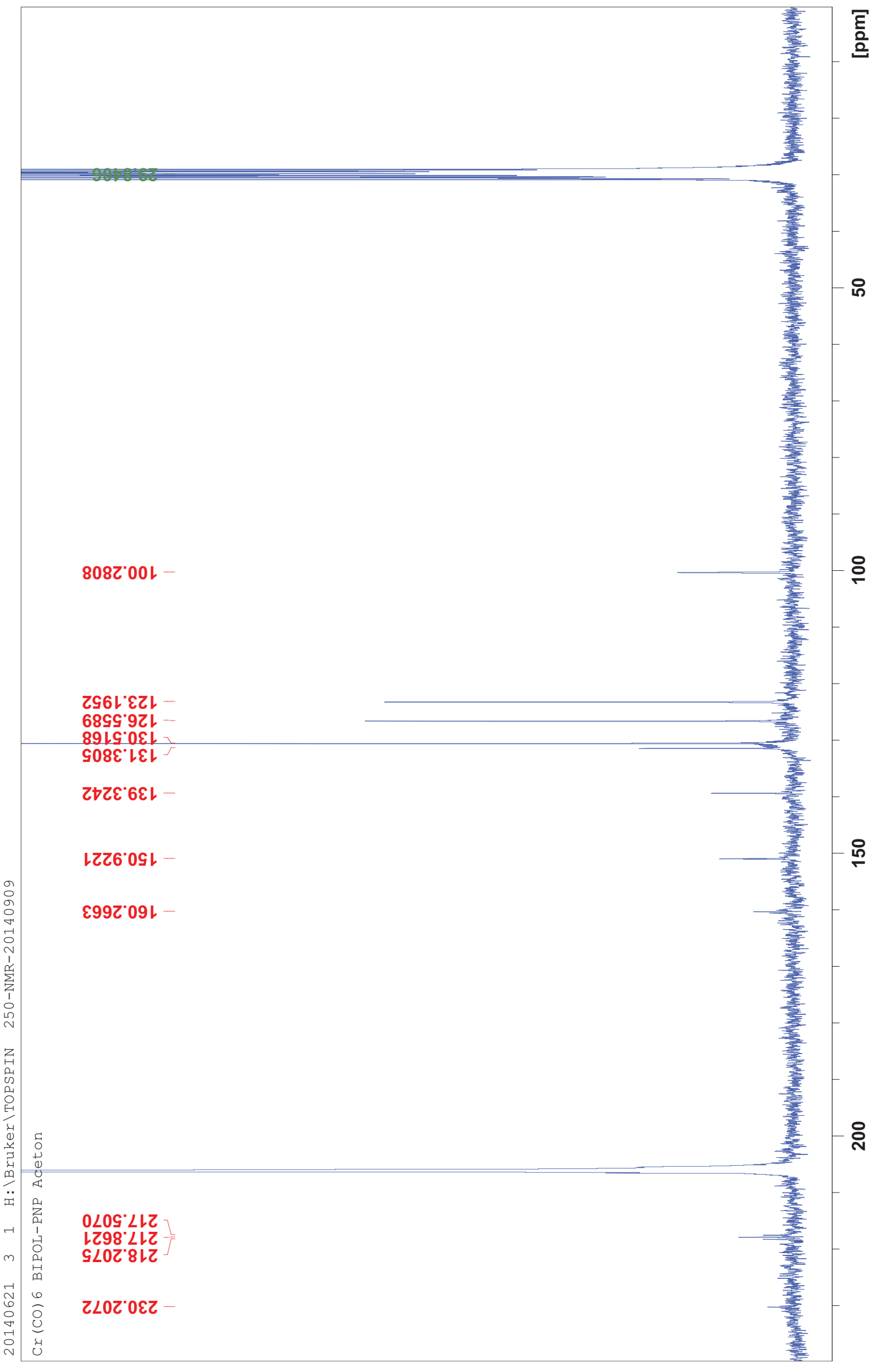




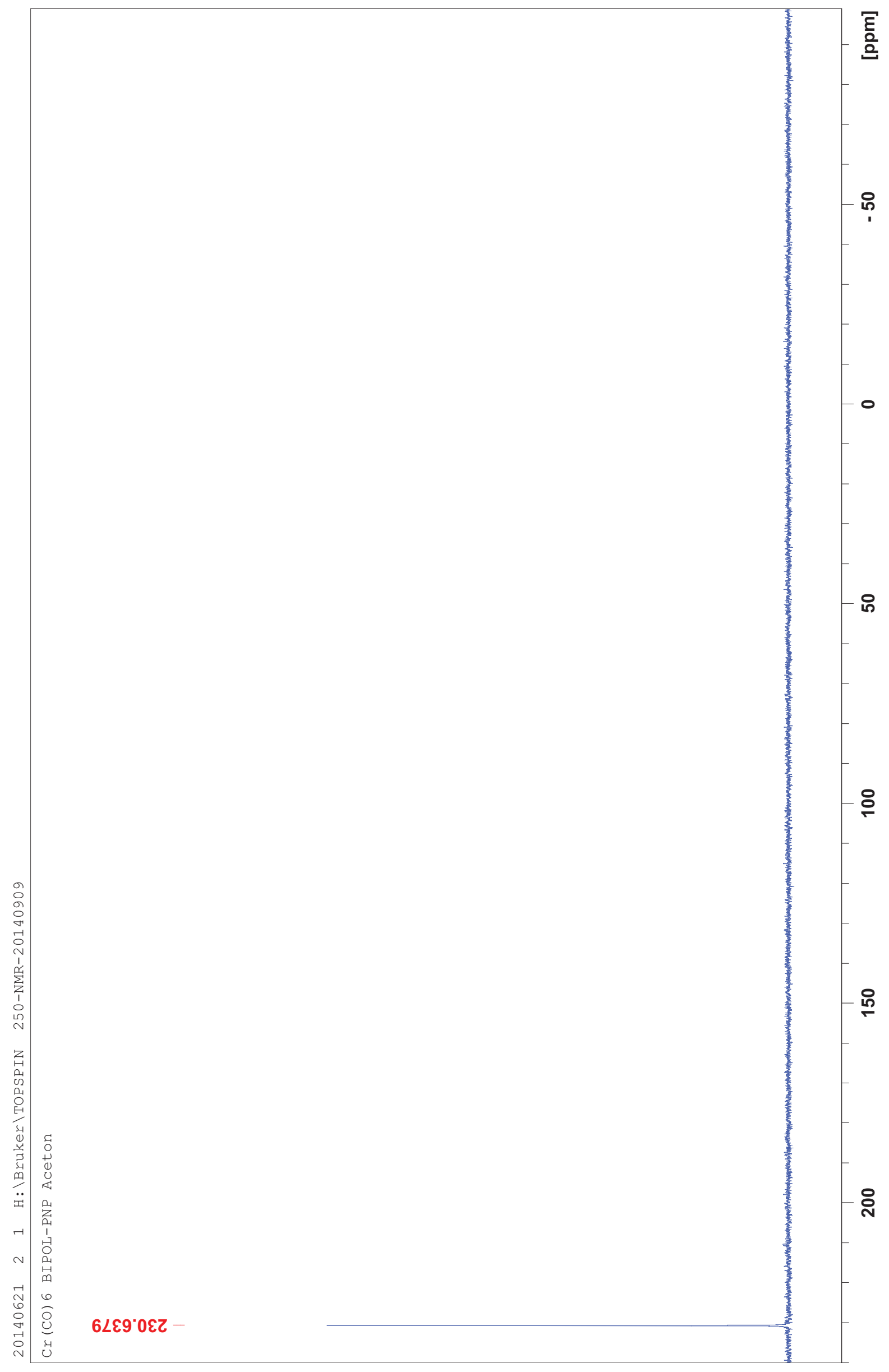




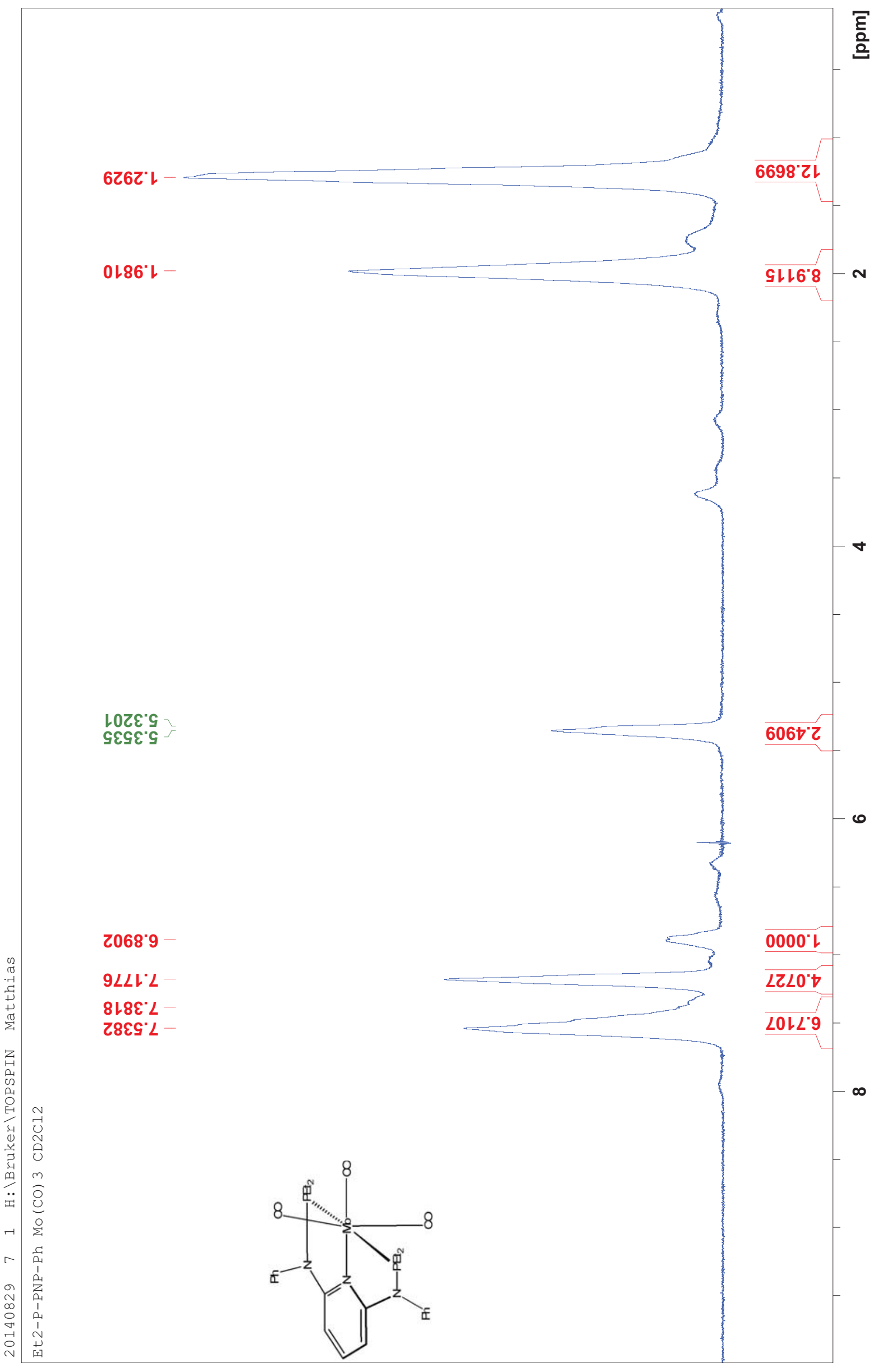




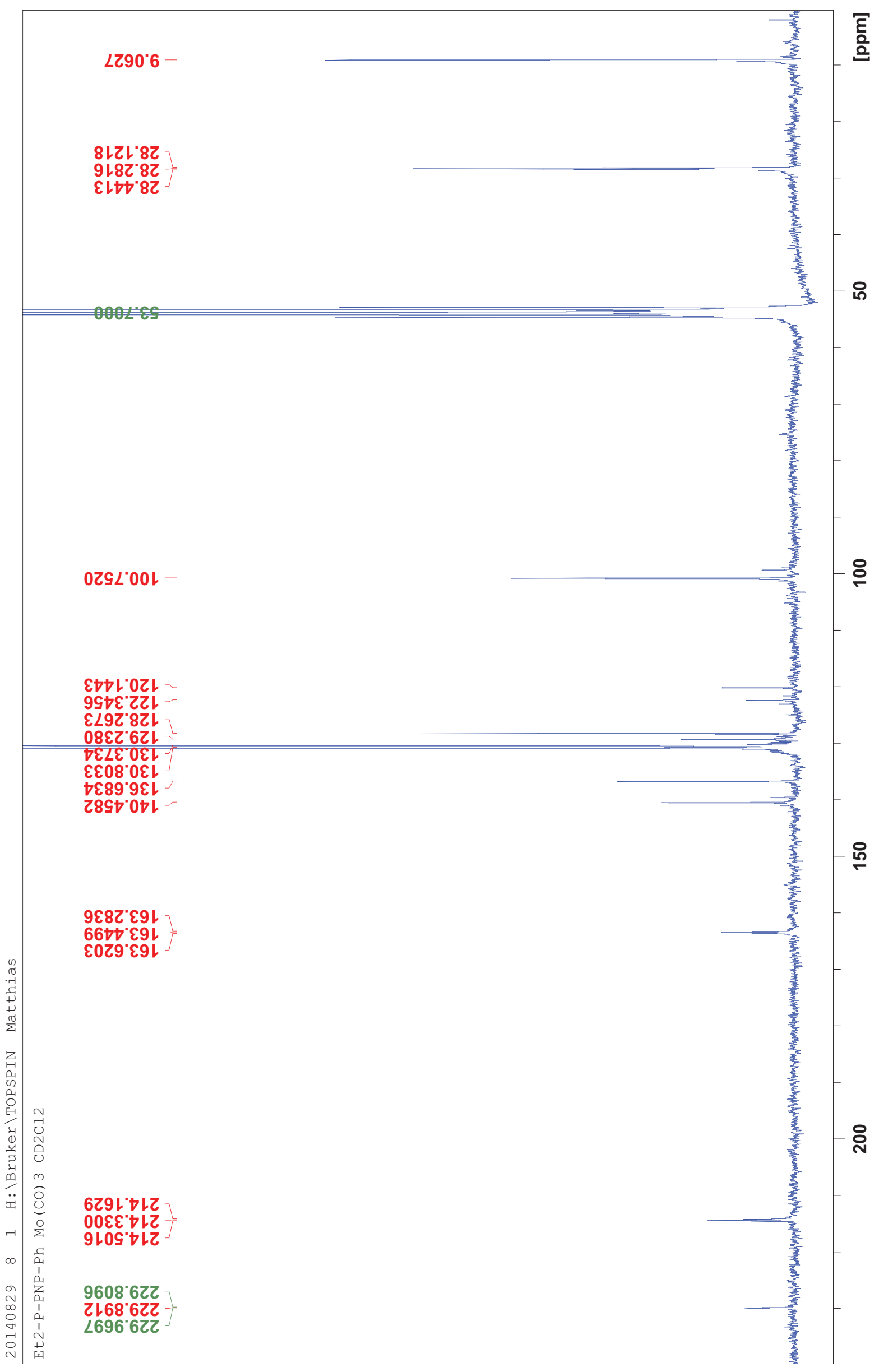




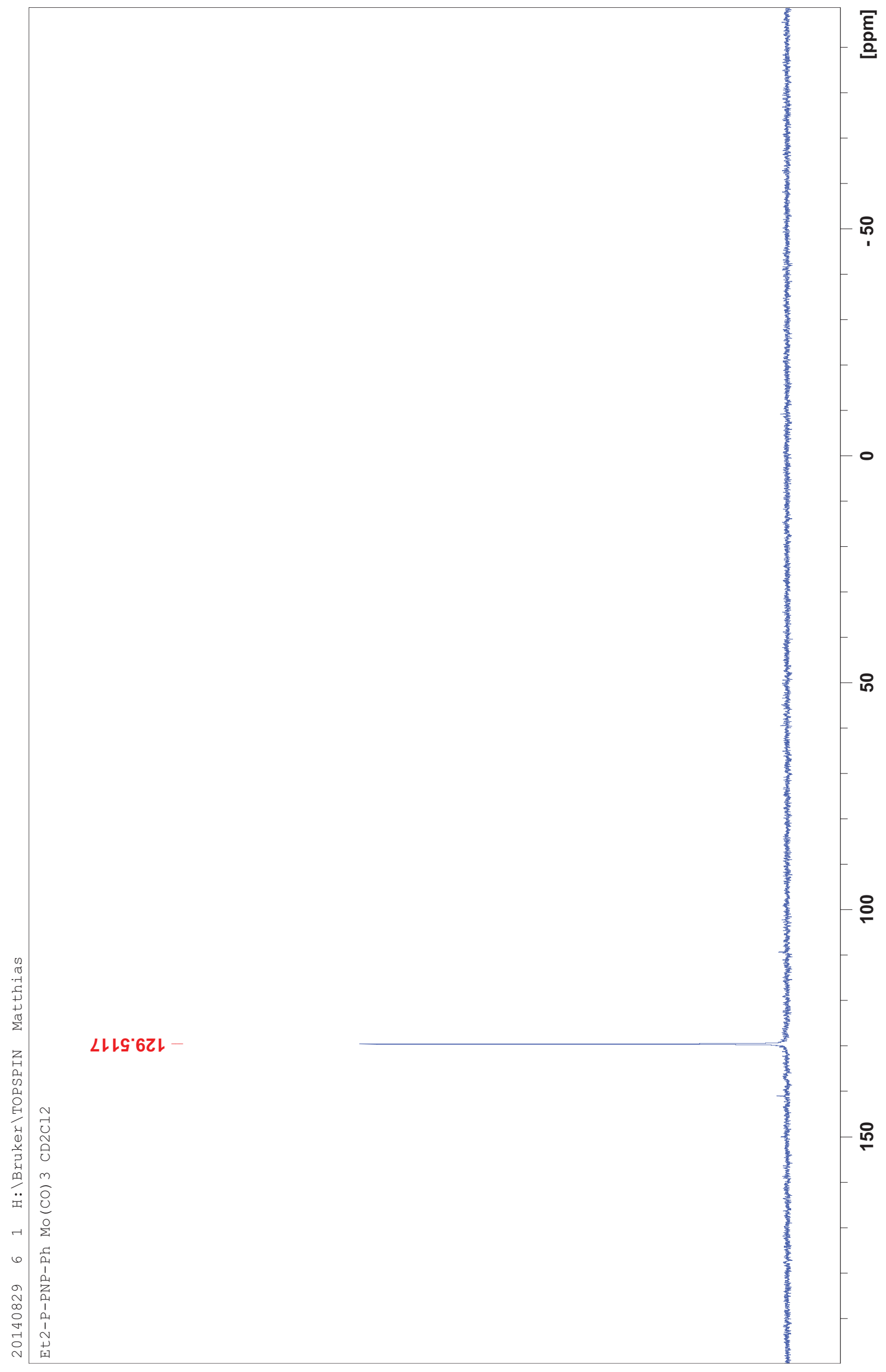




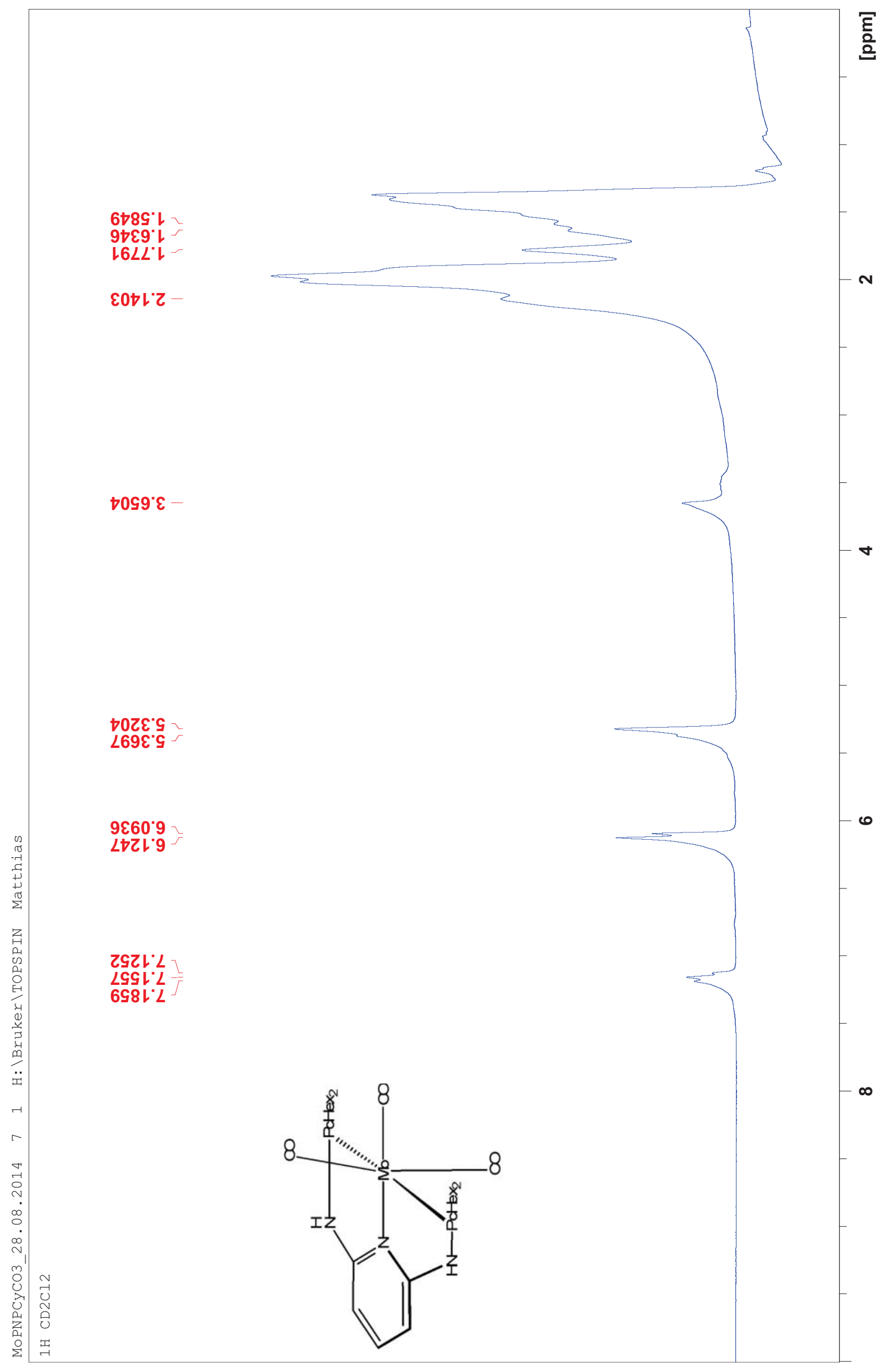




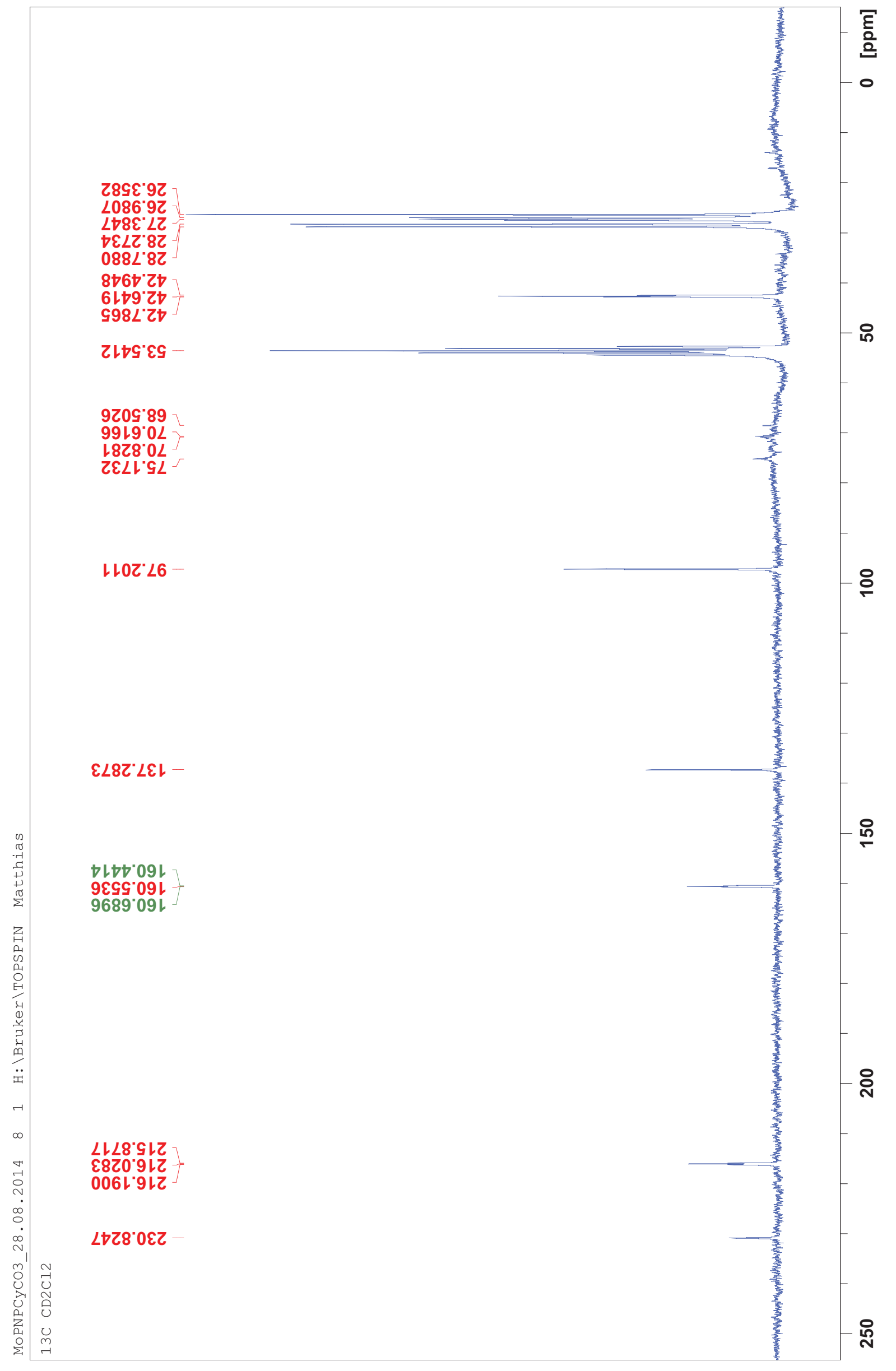




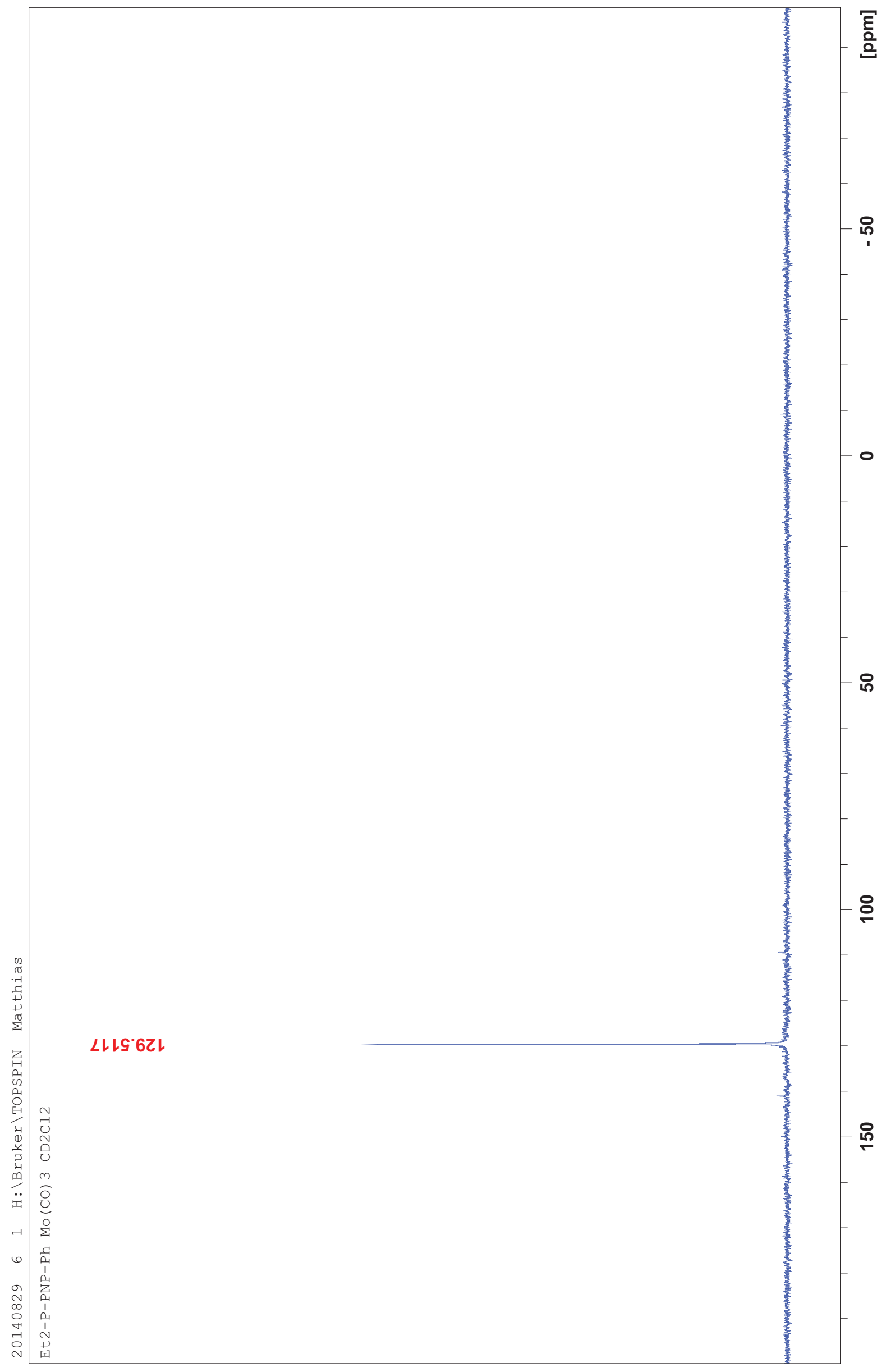




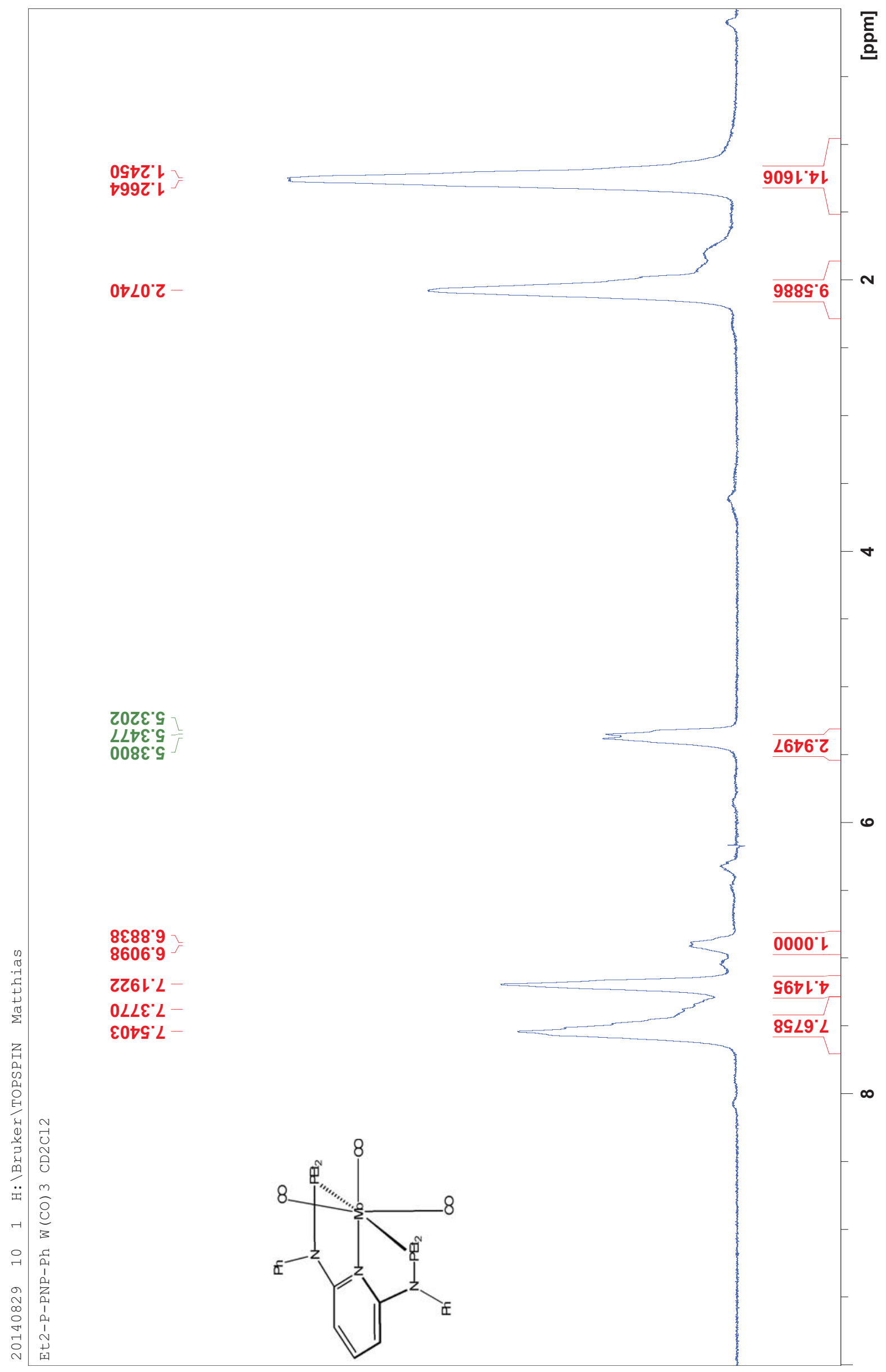




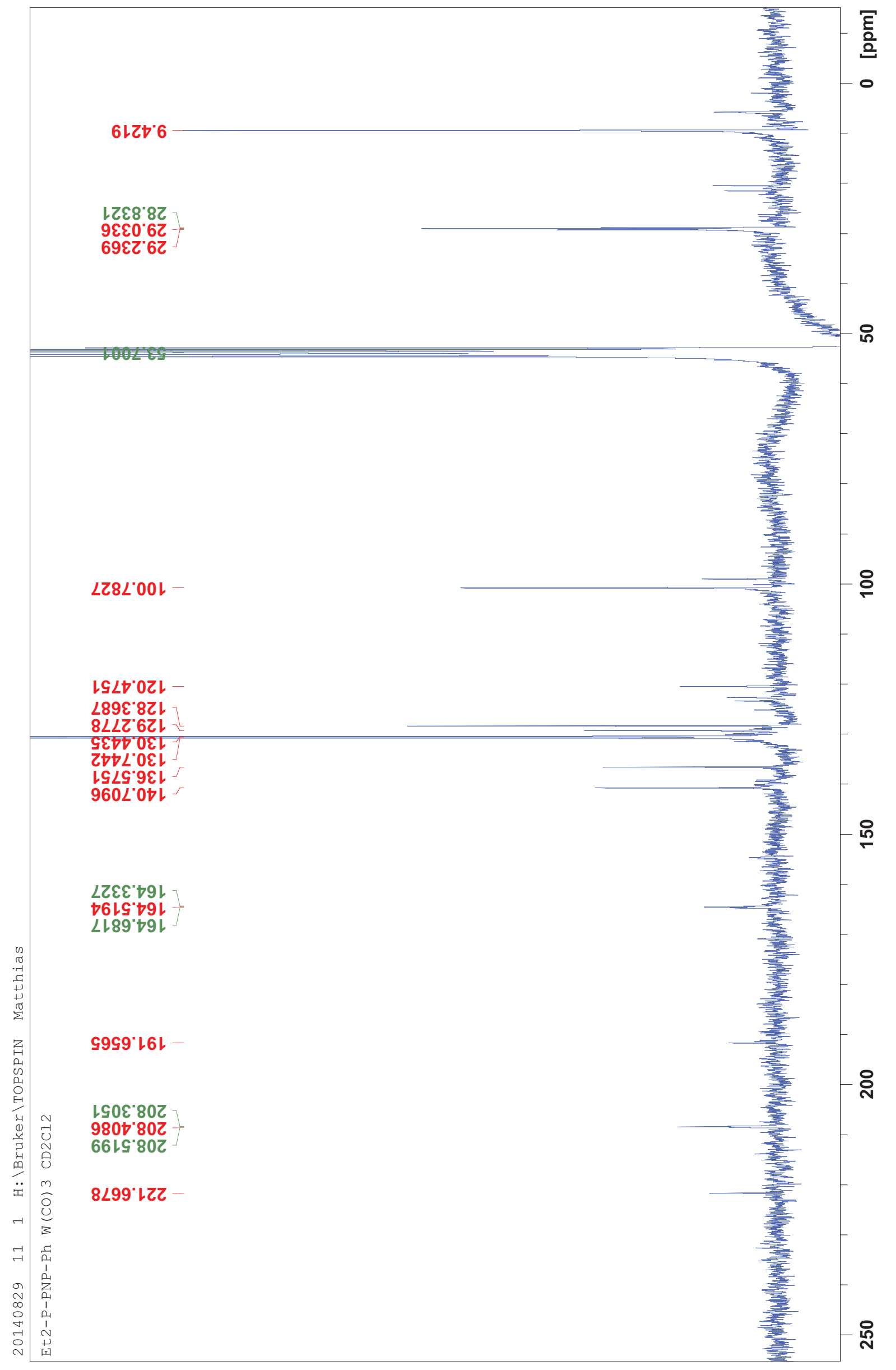




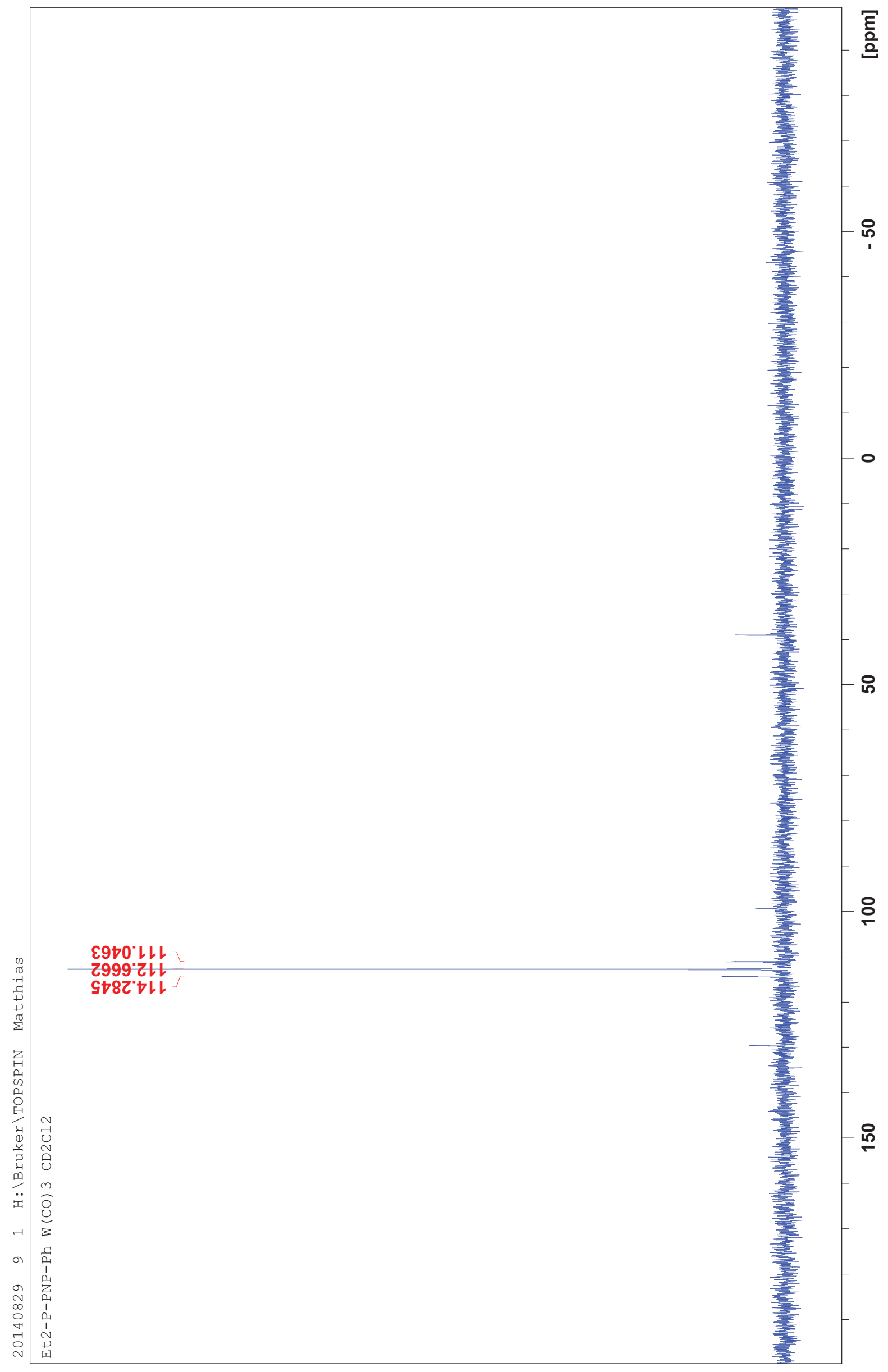




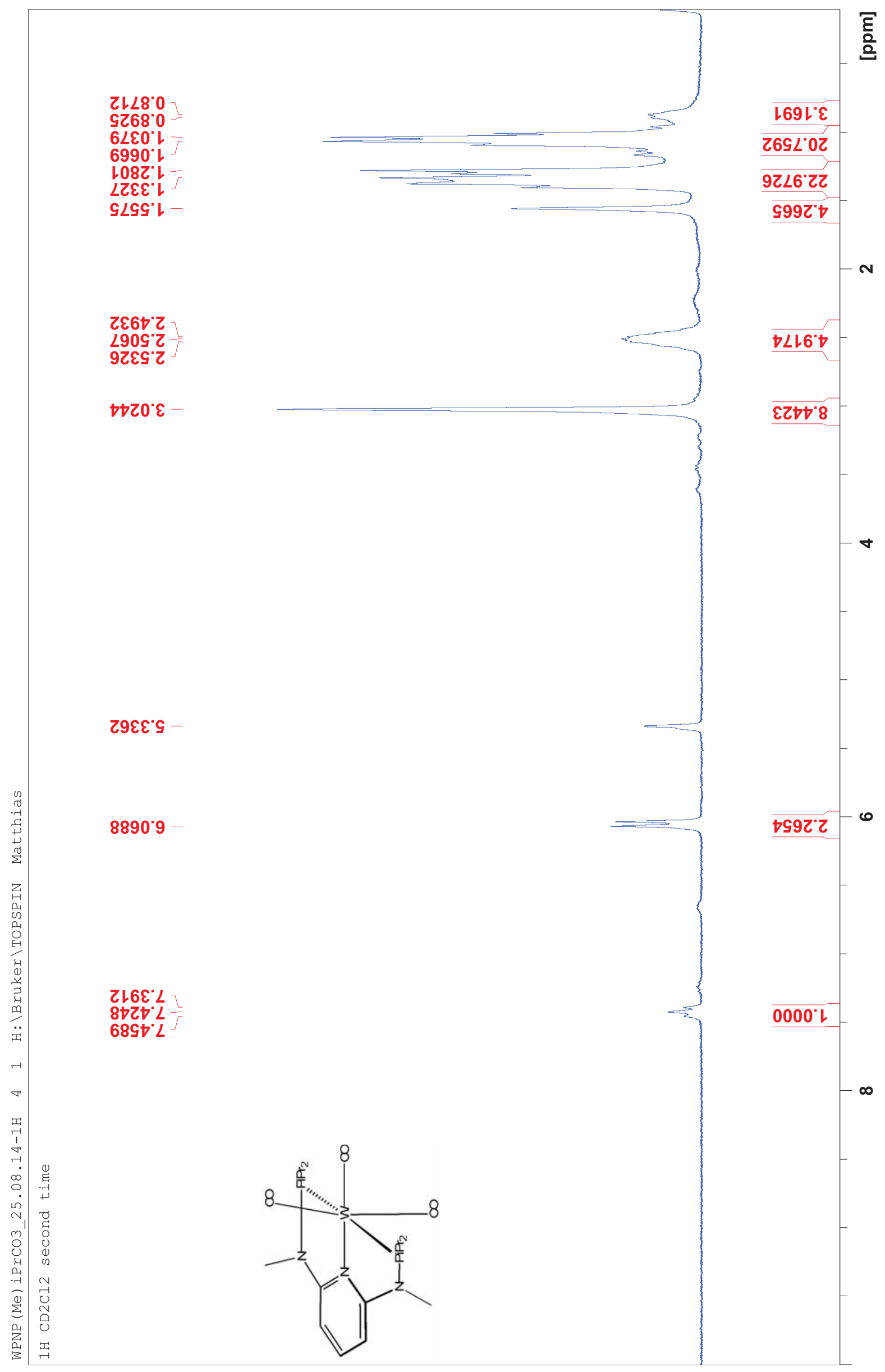




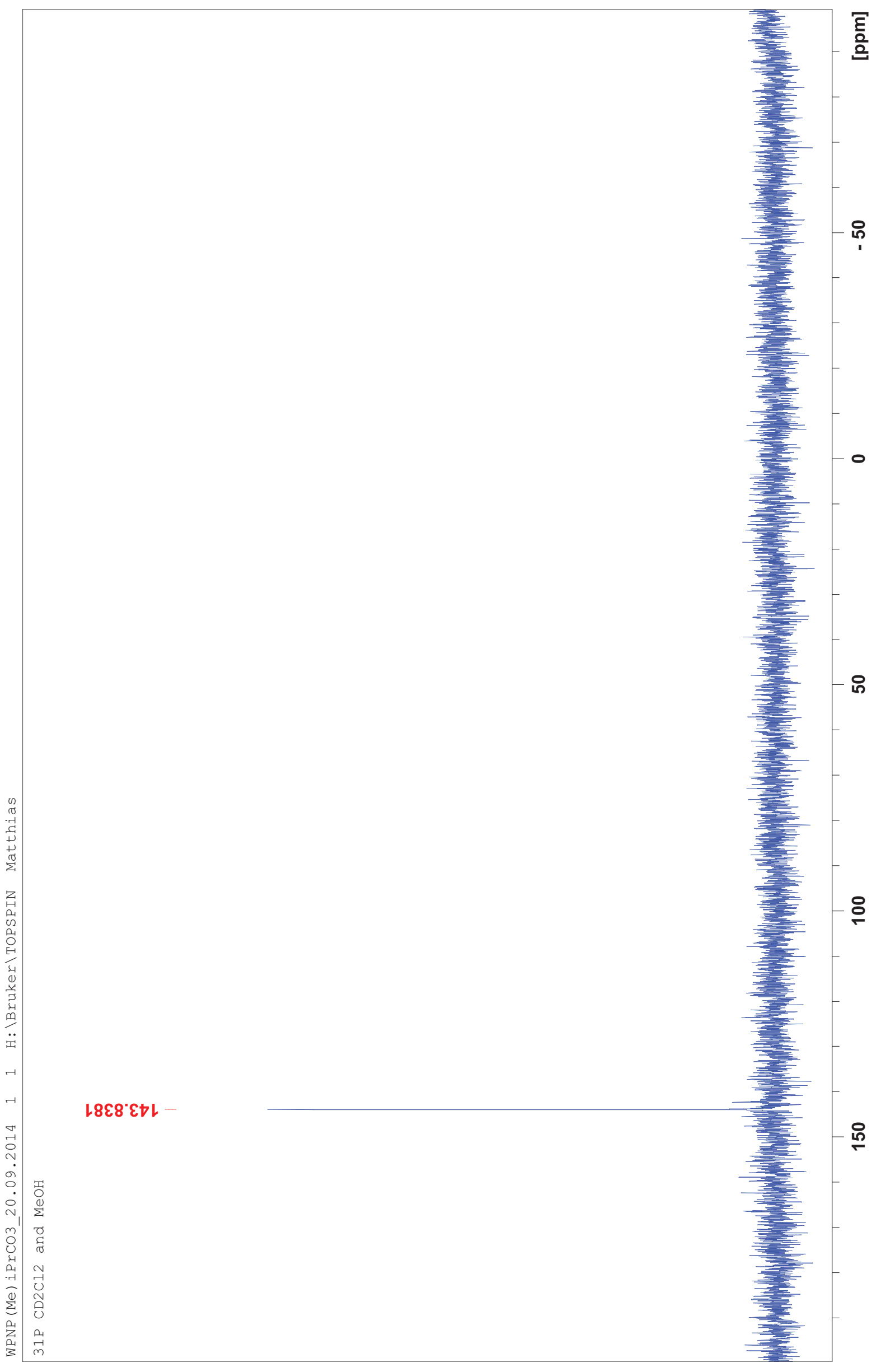




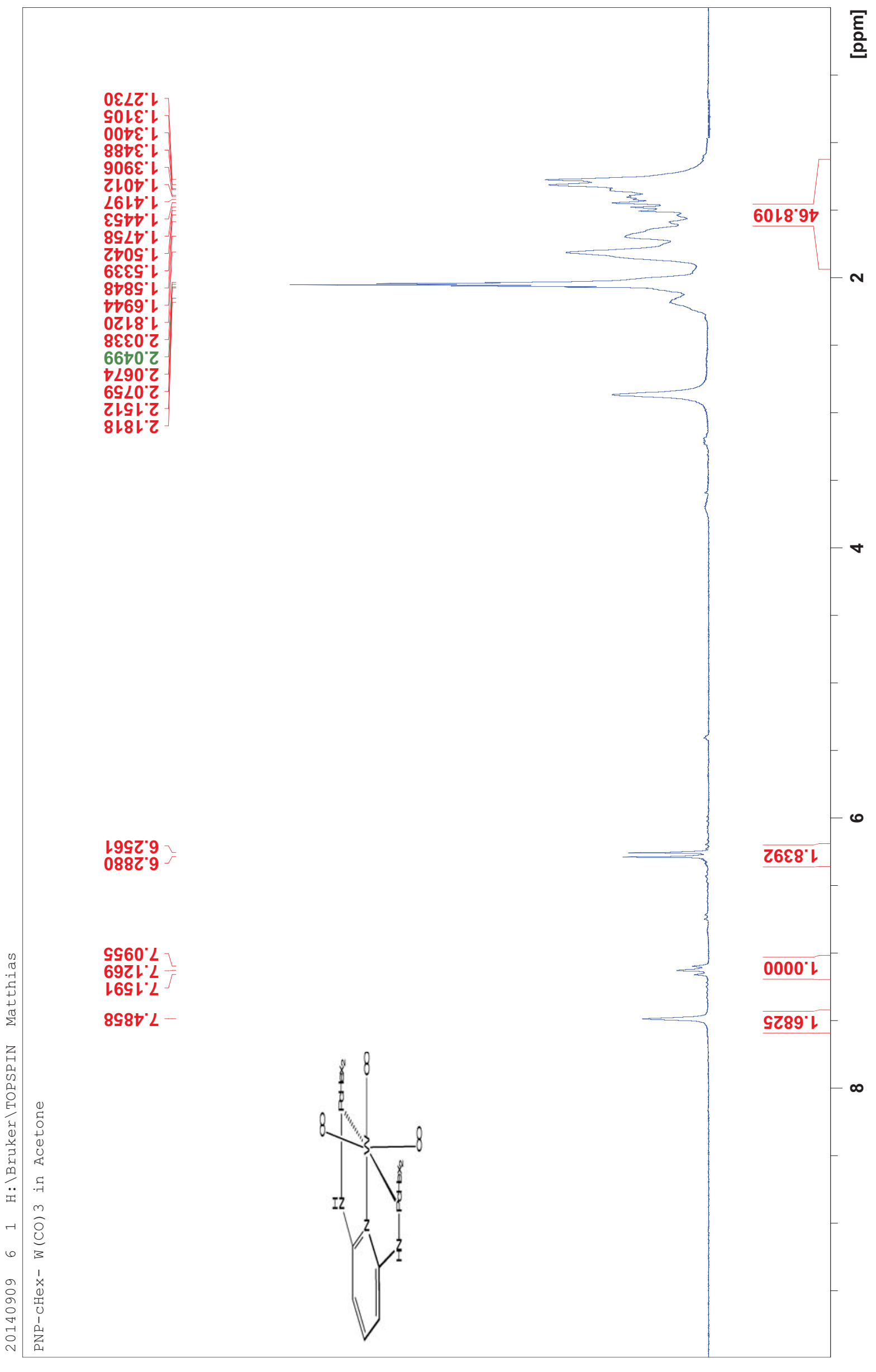




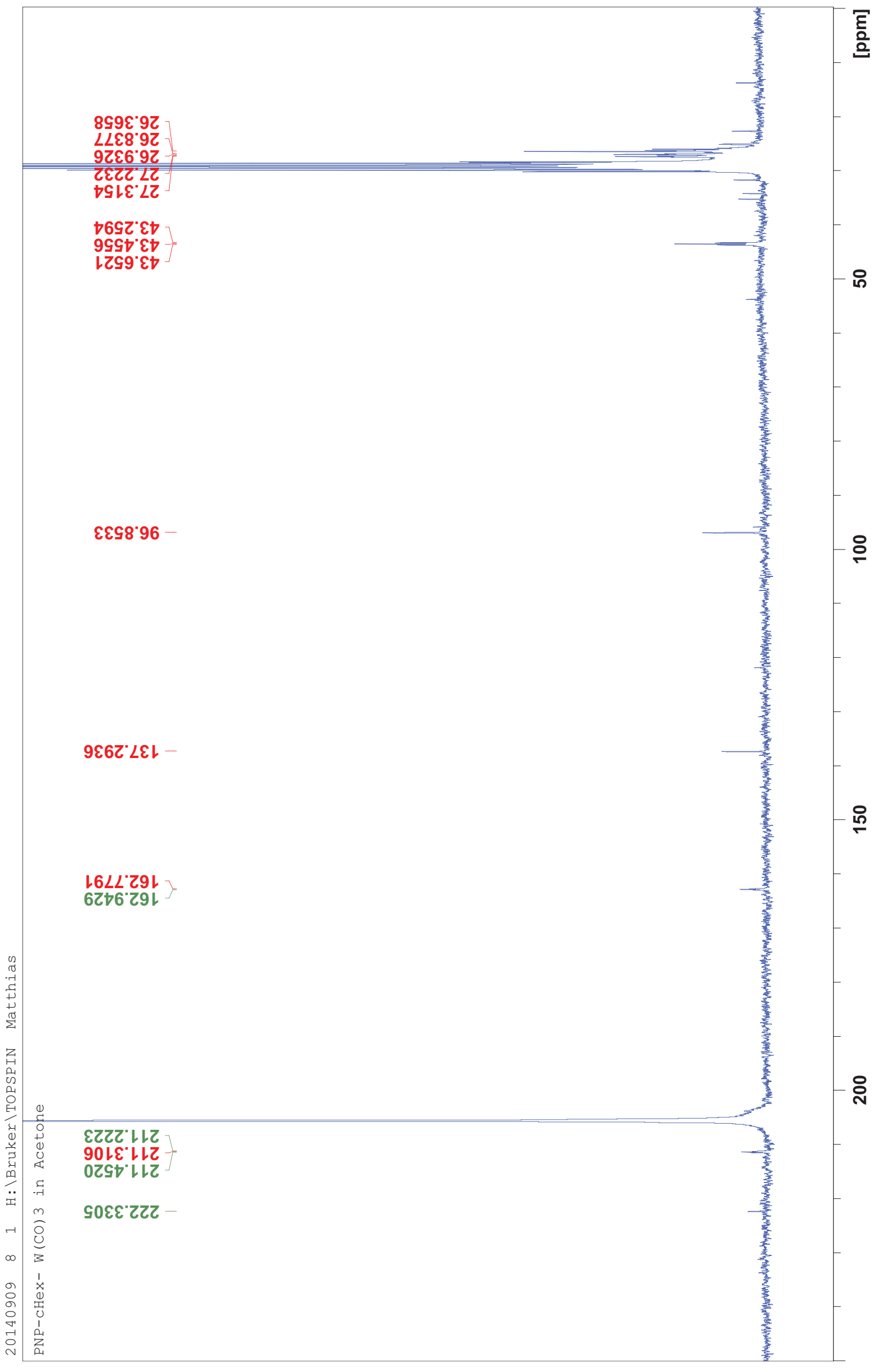




$$
\text { If }
$$




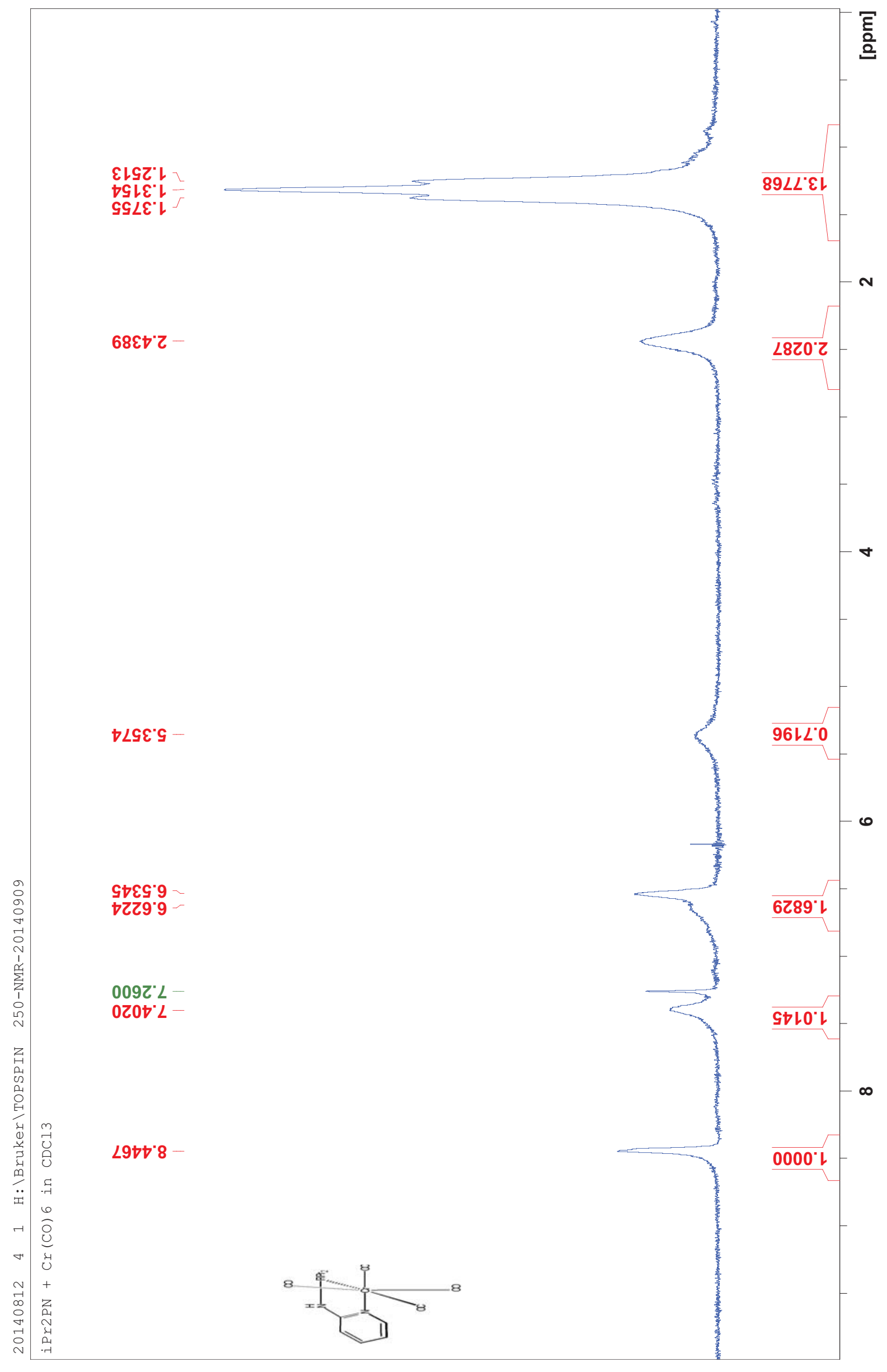




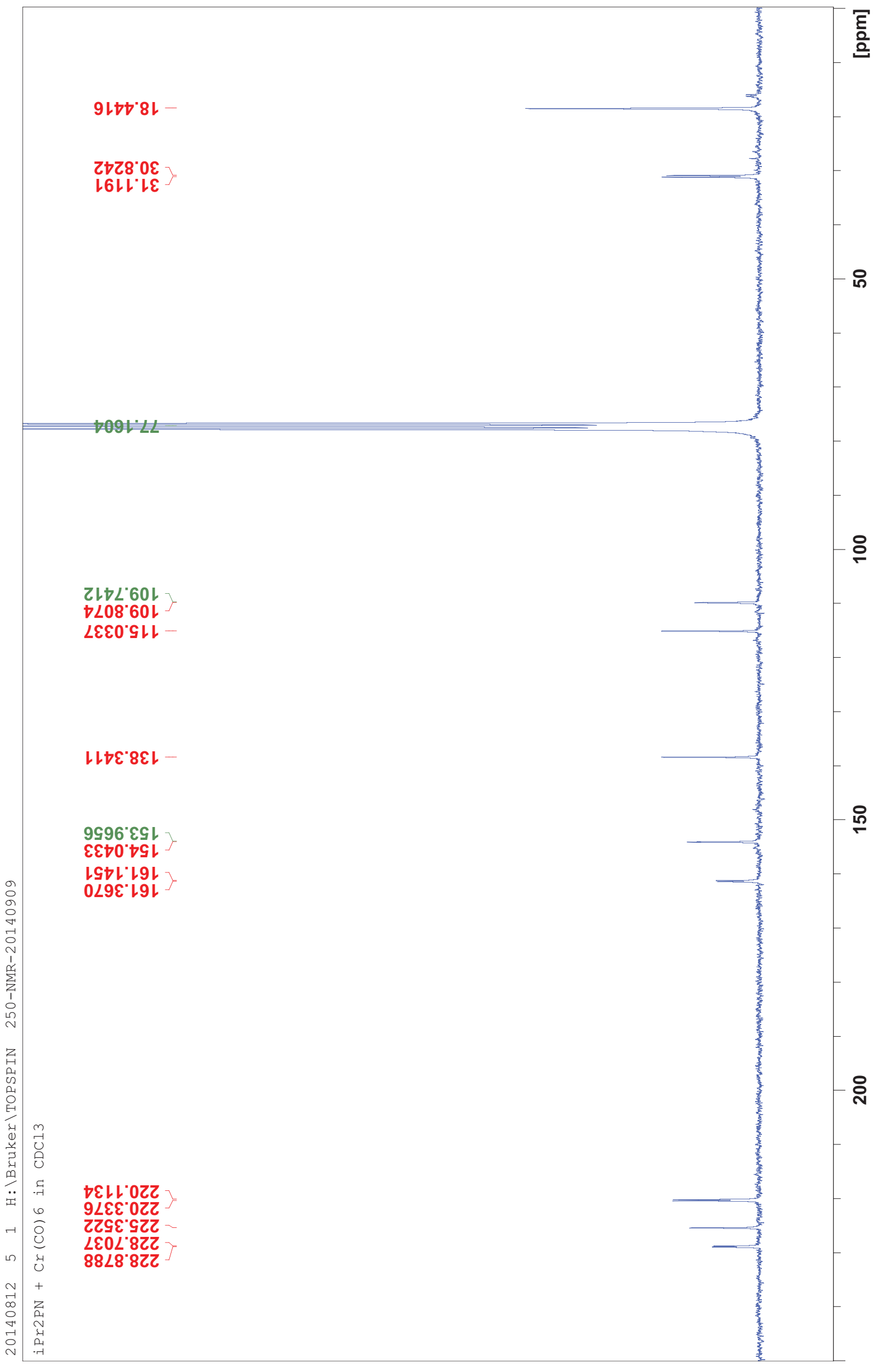




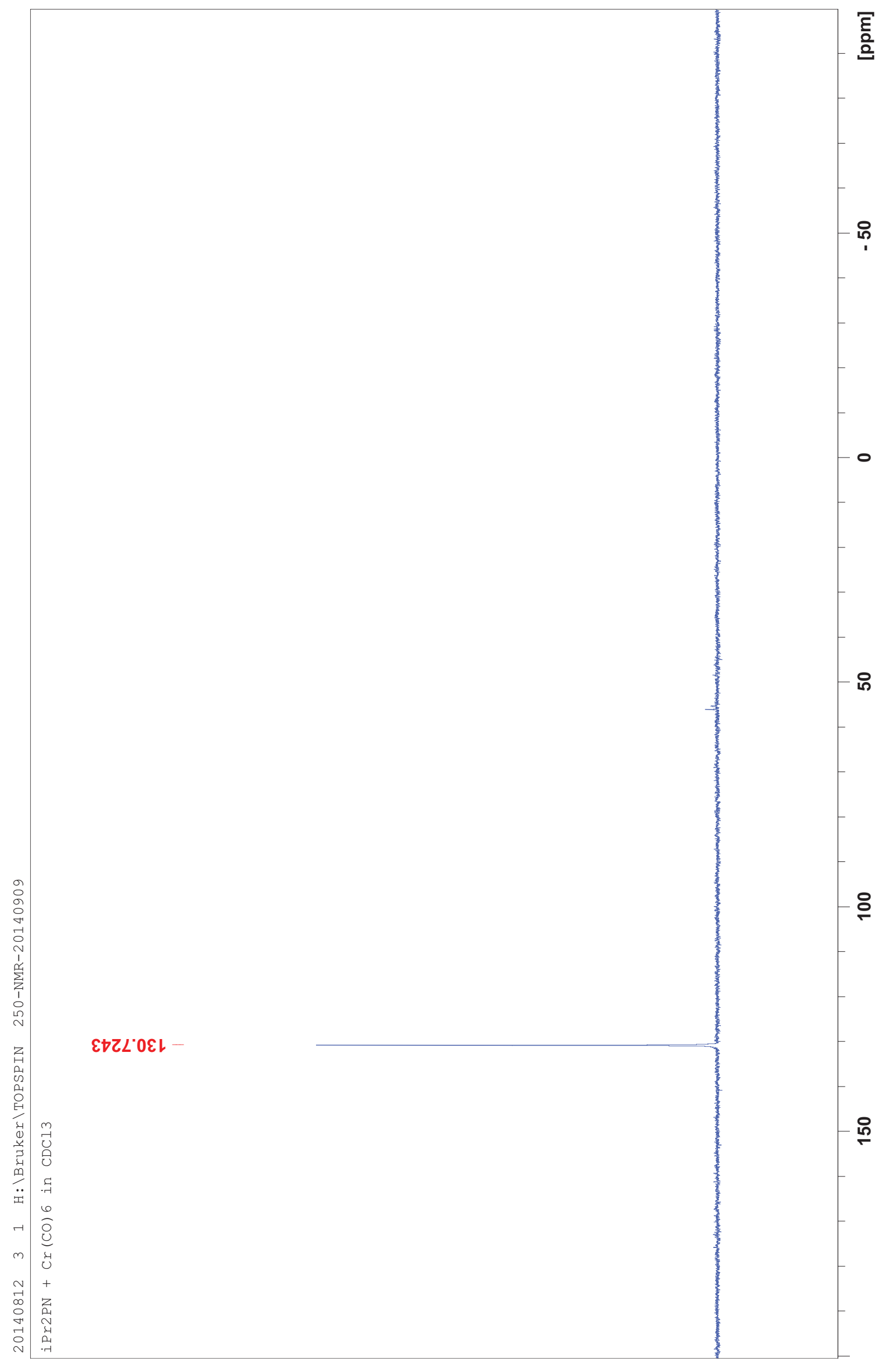




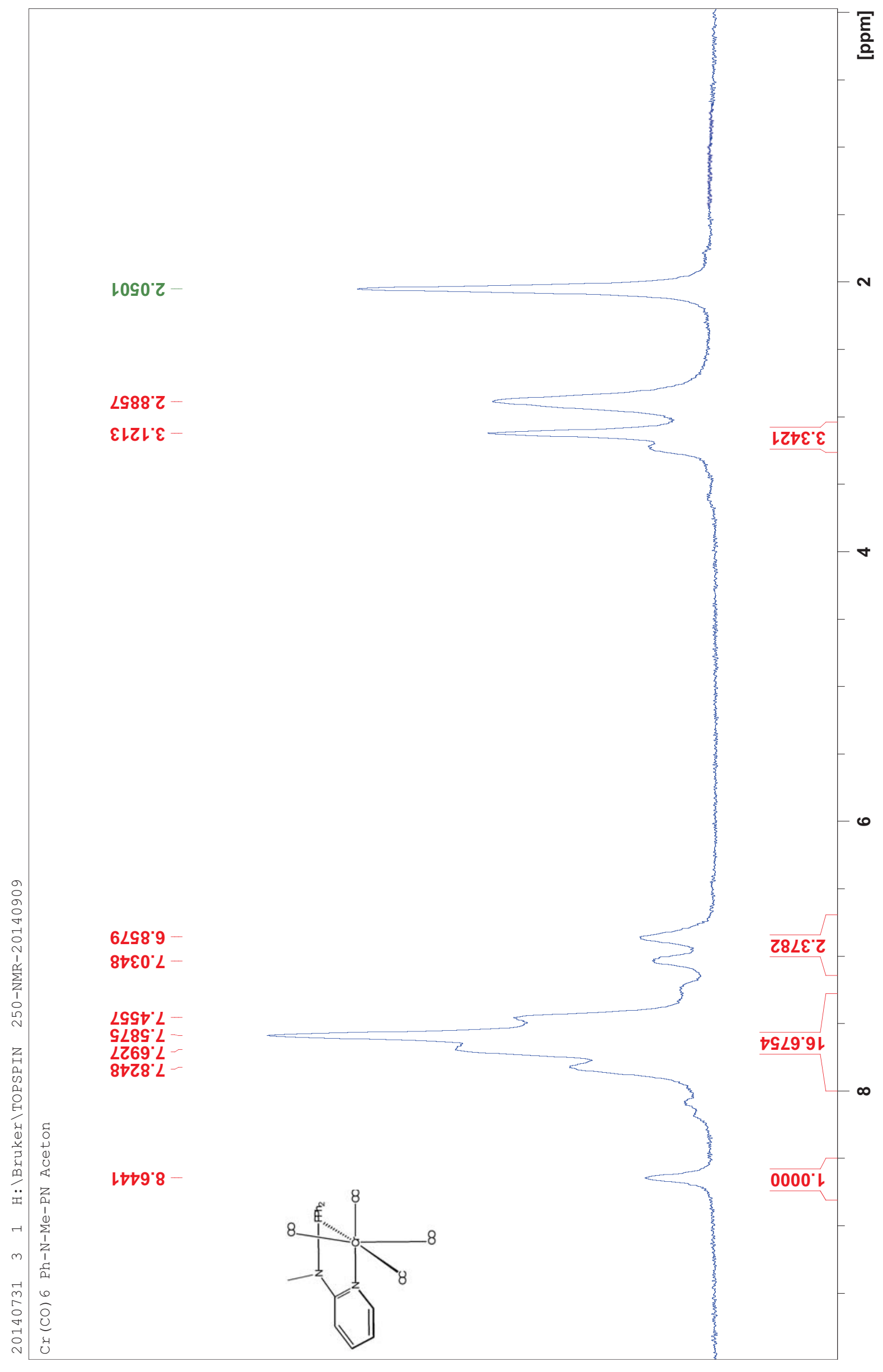




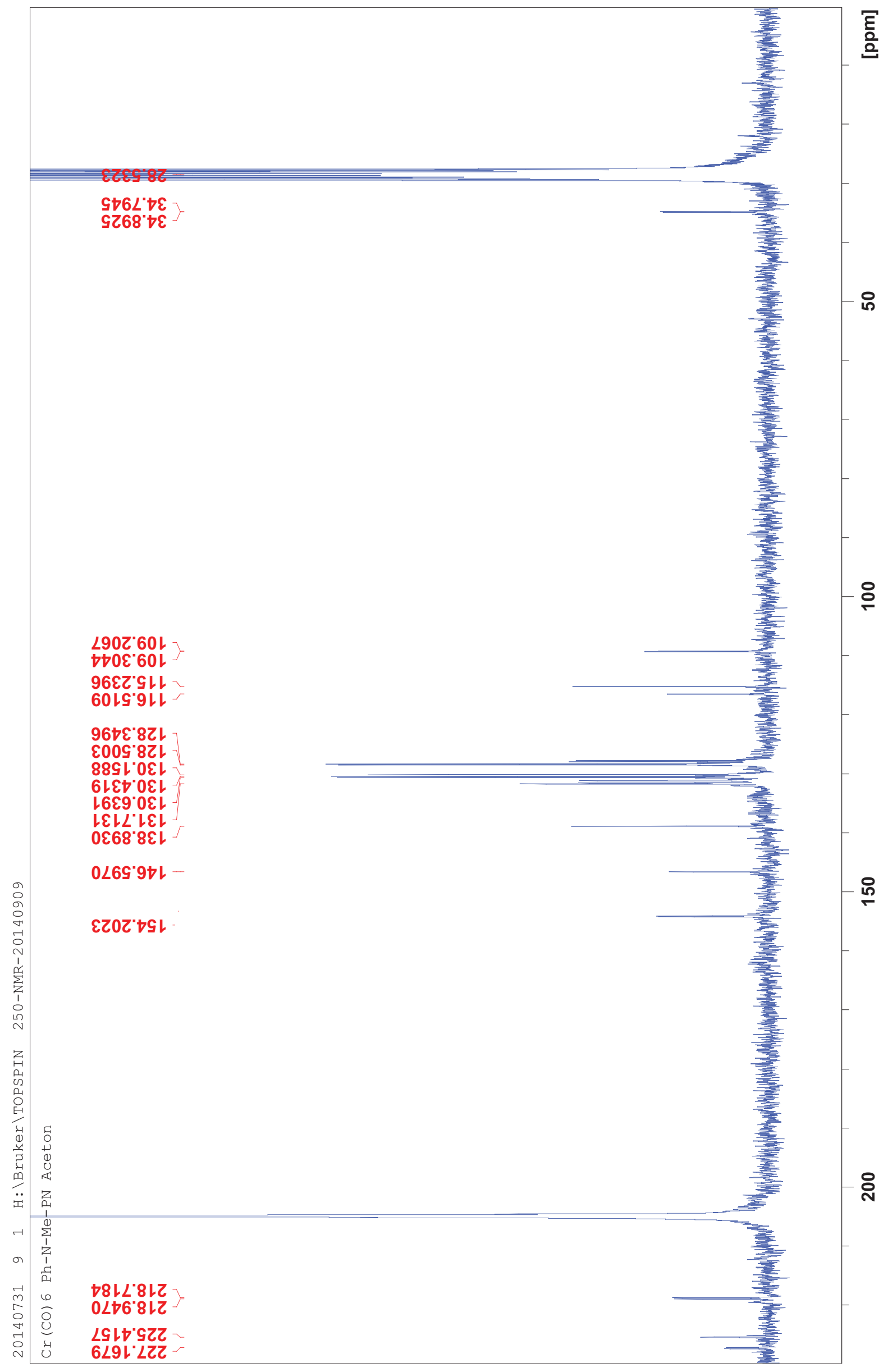




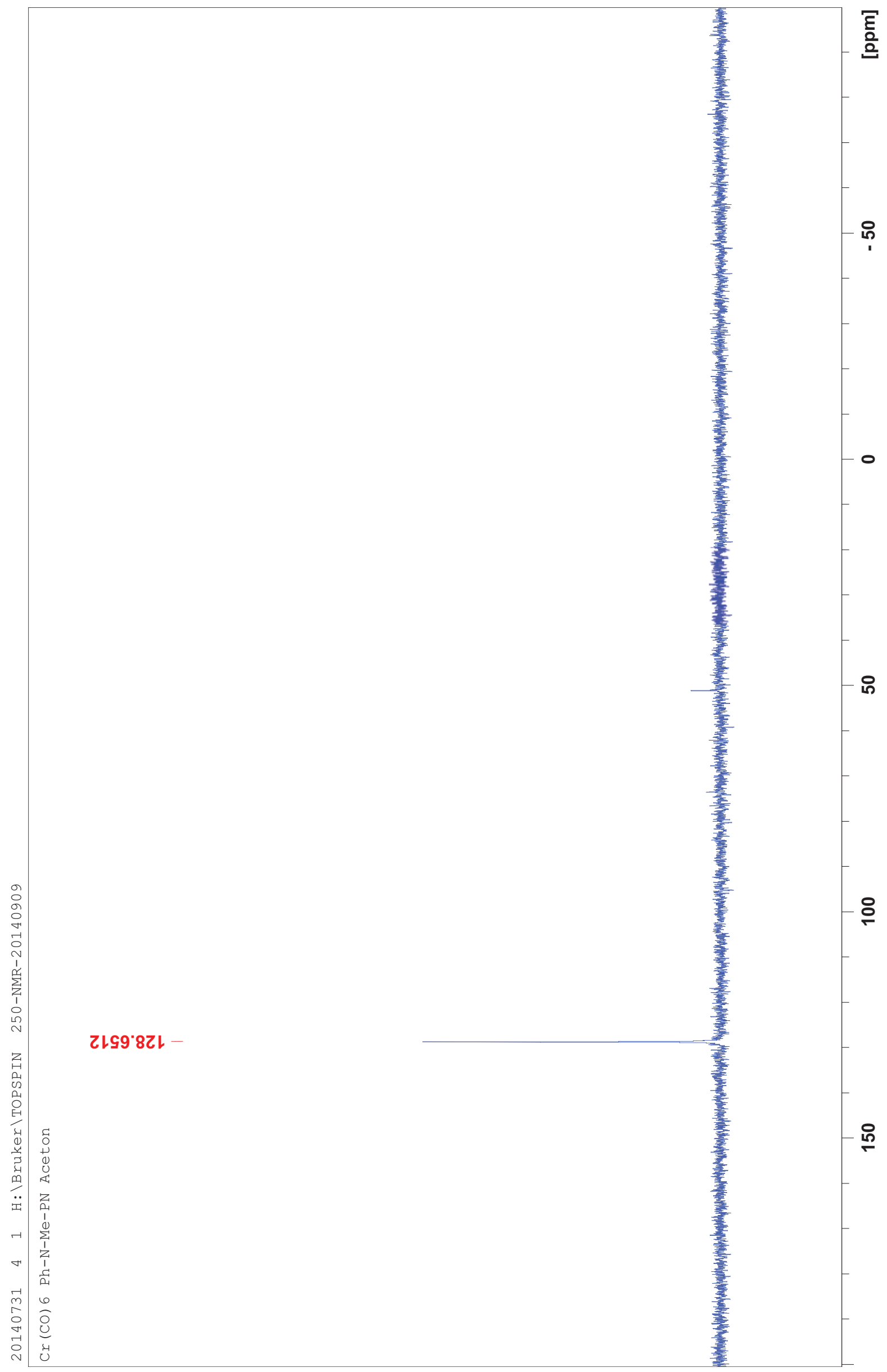




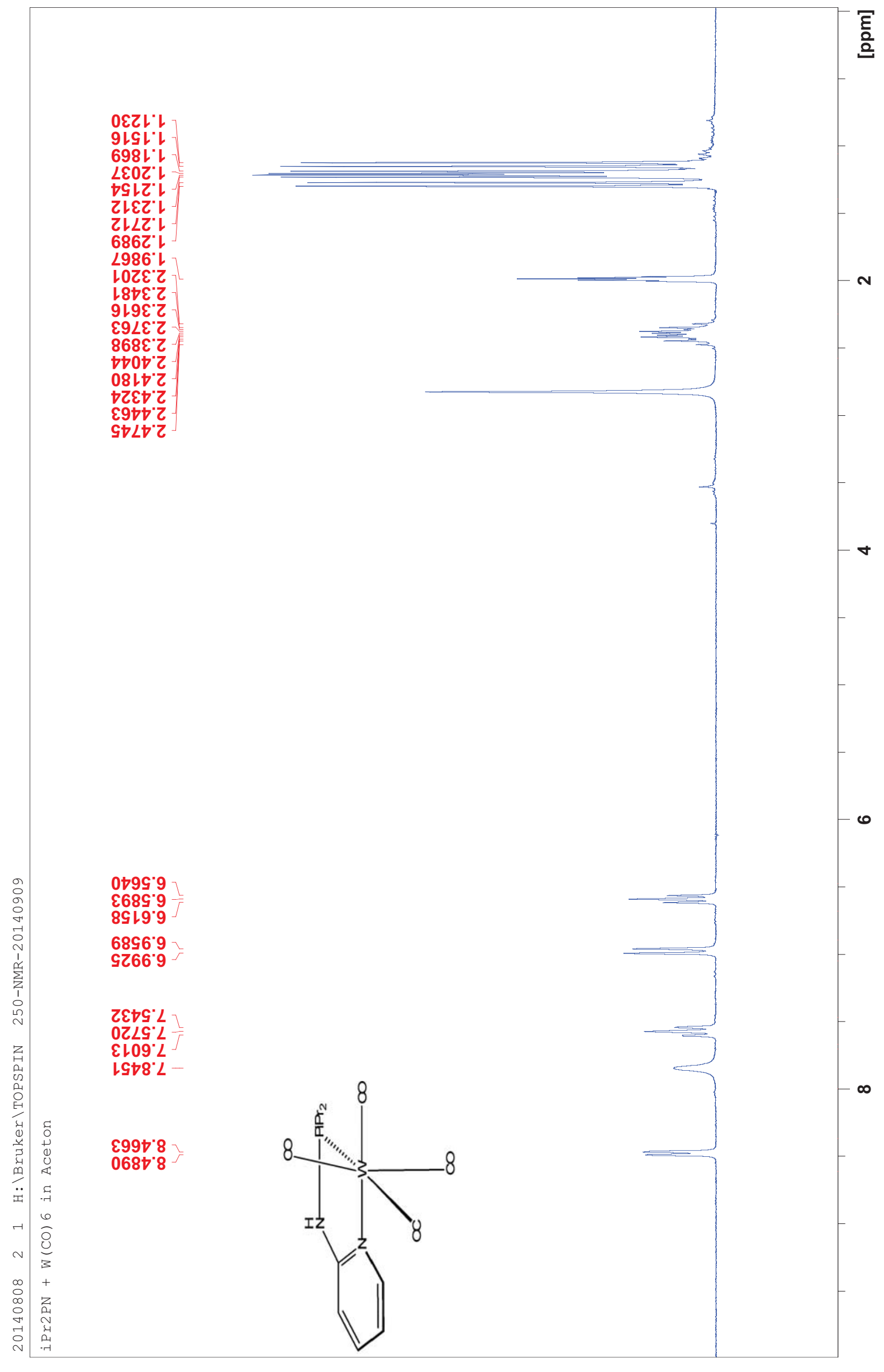




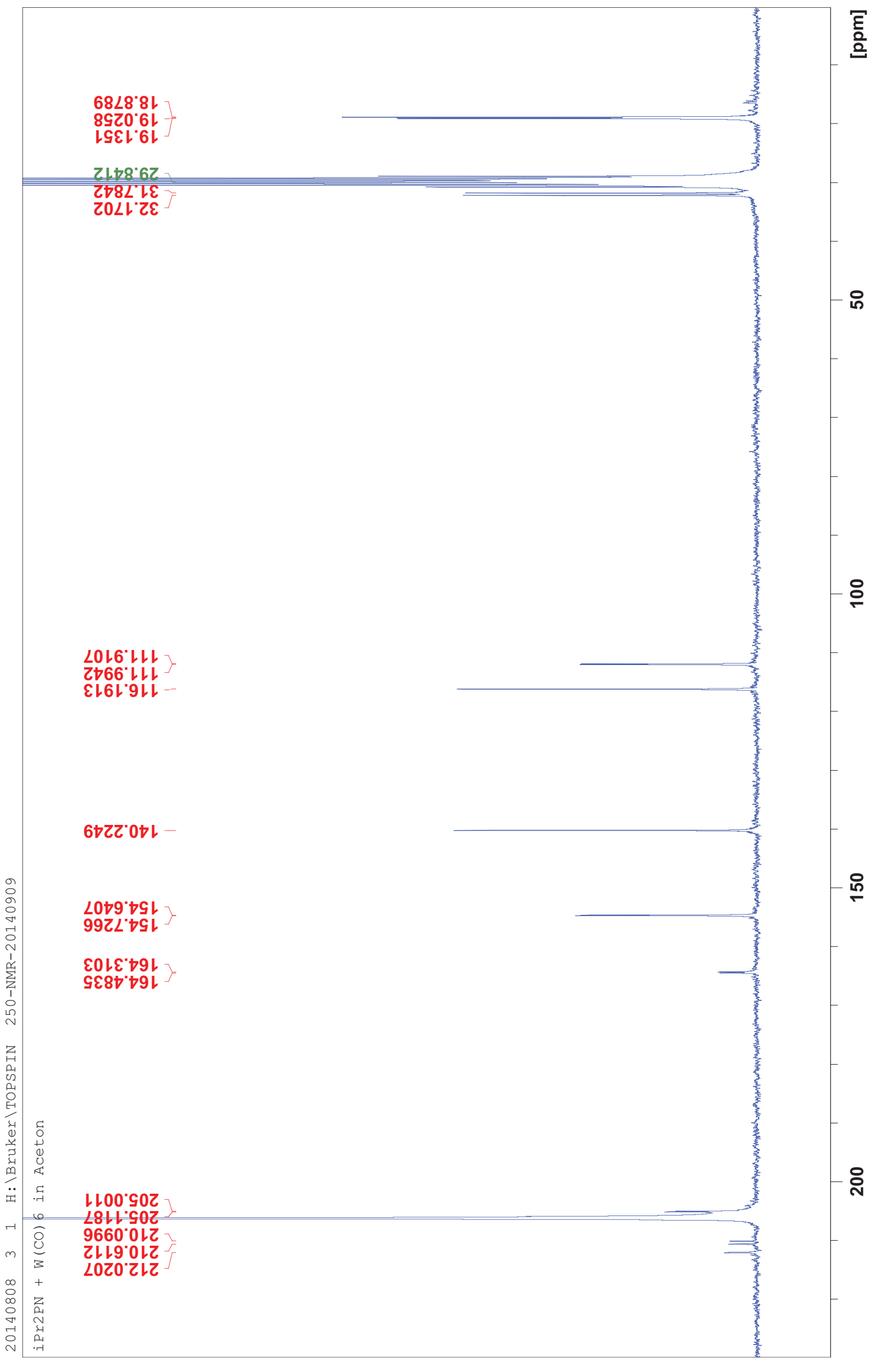




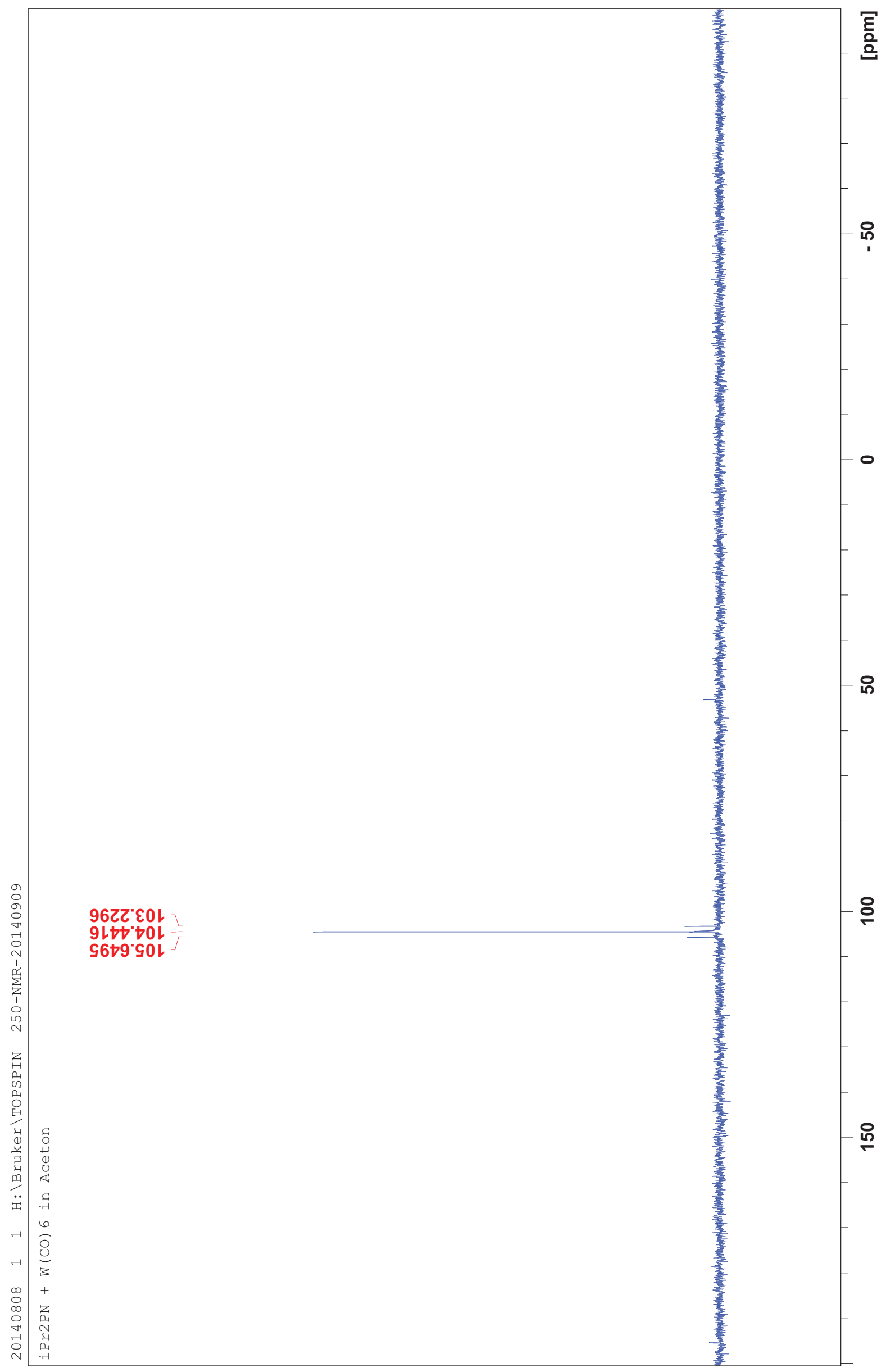

